\title{
LUMINOUS INFRARED GALAXIES WITH THE SUBMILLIMETER ARRAY. I. SURVEY OVERVIEW AND THE CENTRAL GAS TO DUST RATIO
}

\author{
Christine D. Wilson, ${ }^{1,2}$ Glen R. Petitpas, ${ }^{2}$ Daisuke Iono, ${ }^{2,3}$ Andrew J. Baker, ${ }^{4}$ Alison B. Peck, ${ }^{2,5}$ \\ Melanie Krips, ${ }^{2}$ Bradley Warren, ${ }^{1}$ Jennifer Golding, ${ }^{1}$ Adam Atkinson, ${ }^{1}$ Lee Armus, 6 \\ T. J. Cox, ${ }^{2}$ Paul Ho, ${ }^{2,7}$ Mika Juvela, ${ }^{8}$ Satoki Matsushita, ${ }^{7}$ J. Christopher Minos, ${ }^{9}$ \\ Ylva Pihlstrom, ${ }^{10}$ and Min S. Yun ${ }^{11}$ \\ Received 2008 March 20; accepted 2008 June 7
}

\begin{abstract}
We present new data obtained with the Submillimeter Array for a sample of 14 nearby luminous and ultraluminous infrared galaxies. The galaxies were selected to have distances $D_{L}<200 \mathrm{Mpc}$ and far-infrared luminosities $\log L_{\mathrm{FIR}}>11.4$. The galaxies were observed with spatial resolutions of order $1 \mathrm{kpc}$ in the $\mathrm{CO} J=3-2, \mathrm{CO} J=2-1$, ${ }^{13} \mathrm{CO} J=2-1$, and $\mathrm{HCO}^{+} J=4-3$ lines as well as the continuum at $880 \mu \mathrm{m}$ and $1.3 \mathrm{~mm}$. We have combined our CO and continuum data to measure an average gas-to-dust mass ratio of $120 \pm 28$ (rms deviation 109) in the central regions of these galaxies, very similar to the value of 150 determined for the Milky Way. This similarity is interesting given the more intense heating from the starburst and possibly accretion activity in the luminous infrared galaxies compared to the Milky Way. We find that the peak $\mathrm{H}_{2}$ surface density correlates with the far-infrared luminosity, which suggests that galaxies with higher gas surface densities inside the central kiloparsec have a higher star formation rate. The lack of a significant correlation between total $\mathrm{H}_{2}$ mass and far-infrared luminosity in our sample suggests that the increased star formation rate is due to the increased availability of molecular gas as fuel for star formation in the central regions. In contrast to previous analyses by other authors, we do not find a significant correlation between central gas surface density and the star formation efficiency, as traced by the ratio of far-infrared luminosity to nuclear gas mass. Our data show that it is the star formation rate, not the star formation efficiency, that increases with increasing central gas surface density in these galaxies.
\end{abstract}

Subject headings: galaxies: individual (Arp 55, Arp 193, Arp 299, IRAS 10565+2448, IRAS 17208-0014, Mrk 231, Mrk 273, NGC 1614, NGC 2623, NGC 5331, NGC 5257, NGC 5258, NGC 6240, UGC 5101, VV 114) — infrared: galaxies

\section{INTRODUCTION}

Ultraluminous infrared galaxies (ULIRGs) contain the regions of most intense star formation in the local universe. Although their high rates of star formation and accretion appear to be triggered by the merger of two gas-rich galaxies (Sanders et al. 1988a; Veilleux et al. 2002), the detailed physical connection between galaxy mergers and star formation and, in particular, the time evolution of this process is not well understood. Relating numerical hydrodynamical models (Mihos \& Hernquist 1996; Cox et al. 2004) to

\footnotetext{
${ }^{1}$ Department of Physics and Astronomy, McMaster University, Hamilton, ON L8S 4M1 Canada; wilson@physics.mcmaster.ca, bwarren@physics.mcmaster.ca, goldingj@physics.mcmaster.ca, atkinsa@muss.cis.mcmaster.ca.

${ }_{2}$ Harvard-Smithsonian Center for Astrophysics, Cambridge, MA 02138; gpetitpa@cfa.harvard.edu, mkrips@cfa.harvard.edu, tcox@cfa.harvard.edu.

3 National Astronomical Observatory of Japan, 2-21-1 Osawa, Mitaka, Tokyo 181-0015, Japan; d.iono@nao.ac.jp.

4 Department of Physics and Astronomy, Rutgers, The State University of New Jersey, 136 Frelinghuysen Road, Piscataway, NJ 08854-8019; ajbaker@physics .rutgers.edu.

5 Joint ALMA Office, Avda El Golf 40, Piso 18, Santiago, Chile 7550108; apeck@alma.cl.

${ }^{6}$ Spitzer Science Center, California Institute of Technology, Pasadena, CA 91125; lee@ipac.caltech.edu.

7 Academia Sinica Institute of Astronomy and Astrophysics, Taipei 106, Taiwan; pho@asiaa.sinica.edu.tw, satoki@asiaa.sinica.edu.tw.

${ }^{8}$ University of Helsinki Observatory, Finland; mika.juvela@helsinki.fi.

9 Department of Astronomy, Case Western Reserve University, 10900 Euclid Avenue, Cleveland, OH 44106; mihos@case.edu.

10 Department of Physics and Astronomy, University of New Mexico, Albuquerque, NM 87131; ylva@unm.edu.

${ }_{11}$ Department of Astronomy, University of Massachusetts, Amherst, MA 01003;myun@astro.umass.edu.
}

observations is complicated by the difficulty in identifying the precise stage of the merger (Murphy et al. 2001). In addition, while high-resolution imaging has found that most ULIRGs have nuclear separations from $<0.3$ to $48 \mathrm{kpc}$ (Murphy et al. 1996), other strongly interacting galaxies with these nuclear separations which are not ULIRGs have also been found (Braine et al. 2004). These observations suggest that the onset of the intense star formation and accretion which produces a ULIRG is not a simple function of the age of the merger and leaves open the question of whether all luminous infrared galaxies [LIRGs ${ }^{12} ; 11 \leq \log \left(L_{\mathrm{FIR}} / L_{\odot}\right)<12$ ] will pass through a ULIRG phase $\left[\log \left(L_{\mathrm{FIR}} / L_{\odot}\right) \geq 12\right]$ at some point in their evolution.

Local ULIRGs are also important as the closest analogs to the high-redshift submillimeter galaxies (SMGs; Blain et al. 2002); both populations have high infrared luminosities, large amounts of molecular gas (Frayer et al. 1998, 1999; Neri et al. 2003; Greve et al. 2005; Tacconi et al. 2006), and morphological evidence of recent or ongoing mergers (Veilleux et al. 2002; Conselice et al. 2003). Since galaxy merger rates are substantially higher in the early universe (Le Fèvre et al. 2000; Gottlöber et al. 2001), understanding the physical and dynamical properties of nearby ULIRGs is also important for understanding the processes in the early universe which give rise to the very luminous submillimeter galaxy population.

Because molecular gas is the fuel for current and future star formation, the physical properties and distribution of the warm,

\footnotetext{
${ }^{12} L_{\mathrm{FIR}}=4 \pi D_{L}^{2} F_{\mathrm{FIR}} L_{\odot}$, where $F_{\mathrm{FIR}}=1.26 \times 10^{-14}\left(2.58 f_{60}+f_{100}\right) \mathrm{ergs} \mathrm{cm}^{-2}$ $\mathrm{s}^{-1}$ and $f_{60}$ and $f_{100}$ are the IRAS fluxes in janskys at 60 and $100 \mu \mathrm{m}$ (Sanders \& Mirabel 1996).
} 
dense molecular gas are crucial for understanding the processes and timescales controlling star formation in galaxy mergers. Previous high-resolution studies of molecular gas in LIRGs have used primarily the ground-state rotational transition of $\mathrm{CO}$, which is sensitive to gas as cold as $10 \mathrm{~K}$ (Scoville et al. 1991; Downes \& Solomon 1998; Bryant \& Scoville 1999), with a few galaxies observed in the CO $J=2-1$ line (Bryant \& Scoville 1996; Downes \& Solomon 1998; Sakamoto et al. 1999; Tacconi et al. 1999). However, since the $\mathrm{CO} J=3-2$ line traces the warmer and denser gas, it is more likely to be directly associated with the starburst activity and/or fueling of the active galactic nuclei (AGNs) in these galaxies. Indeed, observations of the $\mathrm{CO} J=3-2$ emission in two LIRGs, VV 114 (Iono et al. 2004) and NGC 6090 (Wang et al. 2004), reveal that the large-scale distribution and kinematics of the $\mathrm{CO} J=3-2$ line can be significantly different from those of the $\mathrm{CO} J=1-0$ line.

In this paper, we present new data obtained with the Submillimeter Array (SMA) for a sample of 14 luminous and ultraluminous infrared galaxies in the $\mathrm{CO} J=3-2$, CO $J=2-1$, ${ }^{13} \mathrm{CO} J=2-1$, and $\mathrm{HCO}^{+} J=4-3$ lines. In addition, we present new high-resolution observations of continuum emission at $880 \mu \mathrm{m}$ and $1.3 \mathrm{~mm}$, which allow us to study the dust properties in the central kiloparsec of the galaxies. This SMA legacy survey aims to address five broad scientific questions:

1. What are the distributions, kinematics, and physical conditions of dense molecular gas in U/LIRGs? The high-resolution $\mathrm{CO} J=3-2$ data cubes trace the distribution of the warm and dense gas that feeds the starburst (and any accretion) activity in these luminous galaxies. The new SMA CO $J=3-2, J=2-1$, and ${ }^{13} \mathrm{CO} J=2-1$ data can be combined with published $\mathrm{CO}$ $J=1-0$ and $J=2-1$ data for a detailed investigation of the physical properties of the molecular gas using large velocity gradient (LVG; Scoville \& Solomon 1974; Goldreich \& Kwan 1974) and Monte Carlo (Juvela 1997) models as our primary diagnostic tools. The $\mathrm{CO} J=3-2$ kinematics allow us to study the detailed gas dynamics in the inner few hundred pc, yielding measurements of the total enclosed mass and of the local line width that is a parameter in models of disk turbulence. The combination of morphology and kinematics offers clues to the geometry of each merger via comparison of the separation and orientation of the galaxy nuclei with the results from numerical simulations (see, e.g., Mundell et al. 2001 for an analysis of Arp 220).

2. What is the distribution of the dust in U/LIRGs? The $880 \mu \mathrm{m}$ continuum images trace the spatial distribution of the cold and warm $(10-70 \mathrm{~K})$ dust, which reflects both the local rate of star formation activity and the available mass of gas. The submillimeter dust emission is often significantly more compact than that of CO (e.g., Sakamoto et al. 1999, 2006; Meier \& Turner 2001) arising exclusively from deep in the gravitational potential wells of the galactic nuclei. High-resolution continuum images from the SMA can be combined with spectra from the Spitzer Space Telescope (e.g., Armus et al. 2007) to estimate the dust temperature via the mid-infrared to submillimeter spectral energy distribution (SED), the dust mass (including both small and large grains; e.g., Marshall et al. 2007) and, indirectly, the gas mass based on three-dimensional radiative transfer modeling (Juvela \& Padoan 2003). In addition, these spatially resolved SEDs of local U/LIRGS will improve our interpretation of the templates used for determining photometric redshifts of high-redshift submillimeter galaxies (Yun \& Carilli 2002; Aretxaga et al. 2003, 2005).

3. Do the gas properties change as the interaction progresses? Our sample of $14 \mathrm{U} / \mathrm{LIRG}$ covers a range from mid to late merger stages and should be sufficiently large that we can establish a merger sequence through comparison of global morphologies with numerical models (Mihos \& Hernquist 1996; Cox et al. 2004). This data set allows us to determine how the distribution and kinematics of the gas change as a function of physical conditions such as density and temperature, or vice versa, and to correlate those changes with the stage of the merger. This type of detailed gas physics on small scales still poses challenges for numerical simulations. Thus, on large scales, where the dynamics of the system are well described by the models, the numerical simulations can help with the interpretation of the data, while on smaller scales, the data can drive the development of more accurate descriptions for the gas physics in the simulations.

4. How do the properties of the dense gas in local U/LIRGs compare to those of the gas in high-redshift submillimeter galaxies? Armed with a robust local sample of 14 U/LIRGs, we can make a rigorous comparison of the properties of the gas with those in higher redshift galaxies (Greve et al. 2005; Tacconi et al. 2006). Changes in gas characteristics over the age of the universe will reveal important information about the process of star formation and ultimately the formation and evolution of galaxies at early times.

5. What is the origin of nuclear $\mathrm{OH}$ megamasers? Bright $1667 \mathrm{MHz} \mathrm{OH}$ megamaser emission is observed in the nuclei of some LIRGs, including five galaxies in our sample (IRAS 172080014, Mrk 231, Mrk 273, UGC 5101, and Arp 299). These extremely bright masers are promising tracers of dust-obscured star formation and mergers at high redshifts, and could ultimately be used to estimate the merger rate as a function of redshift (Darling \& Giovanelli 2002). However, in order to use $\mathrm{OH}$ megamasers as high-redshift probes, we need to understand whether there is a specific type or stage of merger that leads to $\mathrm{OH}$ maser emission. Whether maser emission occurs is likely governed by the physical, chemical, and kinematic conditions in the molecular gas in the nuclear regions of the mergers. For example, using global $\mathrm{CO}$ and HCN luminosities, Darling (2007) concludes that $\mathrm{OH}$ megamasers are associated with high mean molecular gas densities and high-density gas fractions. Our sample, which contains galaxies with and without megamasers, is well suited for identifying any unique nuclear conditions that produce $\mathrm{OH}$ megamasers in LIRGs.

In this paper, we describe the sample selection, observations, and reduction of the survey data $(\S 2)$. We also examine the gasto-dust mass ratio in the central kiloparsec $(\S 3)$ and correlations between the central gas mass, gas surface density, infrared luminosity, dust temperature, and $\mathrm{CO} J=3-2 / 2-1$ line ratio ( $\S 4)$. A companion paper presents a detailed analysis of the galaxy VV 114 (G. R. Petitpas et al. 2008, in preparation); a detailed analysis of NGC 6240 is given in Iono et al. (2007). Future papers will compare the results from this survey with similar observations of highredshift submillimeter galaxies (Iono et al. 2008), examine the physical properties of the molecular gas by combining molecularline observations with radiative transfer models, use the molecular gas data to place constraints on the origin of the $\mathrm{OH}$ megamaser activity seen in some of the galaxies in our sample, compare the properties of the cold gas and dust as seen with the SMA with the properties of the warm dust derived from Spitzer data, and compare the molecular gas and dust properties with the predictions of numerical simulations to place the galaxies into a merger sequence.

\section{OBSERVATIONS AND DATA REDUCTION}

\subsection{Sample Selection}

For this survey, we selected a sample of LIRGs and ULIRGs with redshifts $z<0.045$ (distances $D_{L}<200 \mathrm{Mpc}$, adopting 
TABLE 1

The Nearby LIRG Sample

\begin{tabular}{|c|c|c|c|}
\hline Galaxy & $\begin{array}{c}\log L_{\mathrm{FIR}} \\
\left(L_{\odot}\right)\end{array}$ & $\begin{array}{c}D_{L} \\
(\mathrm{Mpc})\end{array}$ & $c z$ \\
\hline IRAS $17208-0014 \ldots \ldots \ldots \ldots \ldots$ & 12.41 & 189 & 12835 \\
\hline 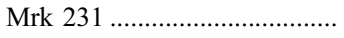 & 12.31 & 179 & 12642 \\
\hline 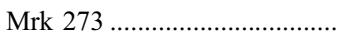 & 12.08 & 166 & 11327 \\
\hline IRAS $10565+2448 \ldots \ldots \ldots \ldots \ldots$ & 11.93 & 191 & 12921 \\
\hline UGC $5101 .$. & 11.87 & 174 & 11809 \\
\hline Arp $299 \ldots \ldots$ & 11.74 & 44 & 3088 \\
\hline 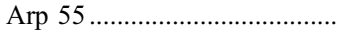 & 11.60 & 173 & 11900 \\
\hline Arp $193 \ldots \ldots \ldots$ & 11.59 & 102 & 7000 \\
\hline NGC $6240 \ldots \ldots$. & 11.54 & 107 & 7339 \\
\hline VV $114 \ldots \ldots \ldots$ & 11.50 & 87 & 6010 \\
\hline 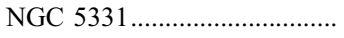 & 11.49 & 145 & 9907 \\
\hline NGC 2623. & 11.48 & 80 & 5535 \\
\hline 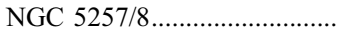 & 11.43 & 99 & 6775 \\
\hline 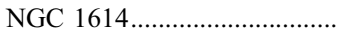 & 11.43 & 69 & 4778 \\
\hline
\end{tabular}

$\left.H_{0}=70 \mathrm{~km} \mathrm{~s}^{-1} \mathrm{Mpc}^{-1}, \Omega_{M}=0.3, \Omega_{\Lambda}=0.7\right)$ and far-infrared luminosities $\log L_{\mathrm{FIR}}>11.4$. The distance limit was chosen to allow spatial resolutions of order $1 \mathrm{kpc}$ or better in all the target galaxies. The luminosity cutoff was chosen to allow us to span a wide range of merger properties and luminosities while still concentrating on the most infrared-luminous nearby galaxies. Out of a total of 39 galaxies above declination $-20^{\circ}$ (Sanders et al. 2003 ) which meet these two criteria, we selected for this survey 14 galaxies with previous interferometric observations in the $\mathrm{CO}$ $J=1-0$ transition. The galaxies observed in this survey are listed in Table 1 and shown in Figure 1. The sample includes five systems in which the two progenitor galaxies are still distinct (Arp 299, Arp 55, VV 114, NGC 5331, and NGC 5257/8); the remaining nine galaxies show a single central concentration.

Compared to the full sample of 39 galaxies, the sample observed with the SMA has a much higher fraction of galaxies with a large far-infrared flux (86\% with $F_{\mathrm{FIR}}>45 \mathrm{Jy}$ compared to $44 \%$ for the full sample). The SMA sample contains a higher proportion of nearby galaxies ( $29 \%$ with $z<0.021$ compared to $15 \%$ for the full sample), although the mean distance of the SMA sample is only $4 \%$ smaller than the mean distance of the full sample. The SMA sample also has a higher proportion of more luminous

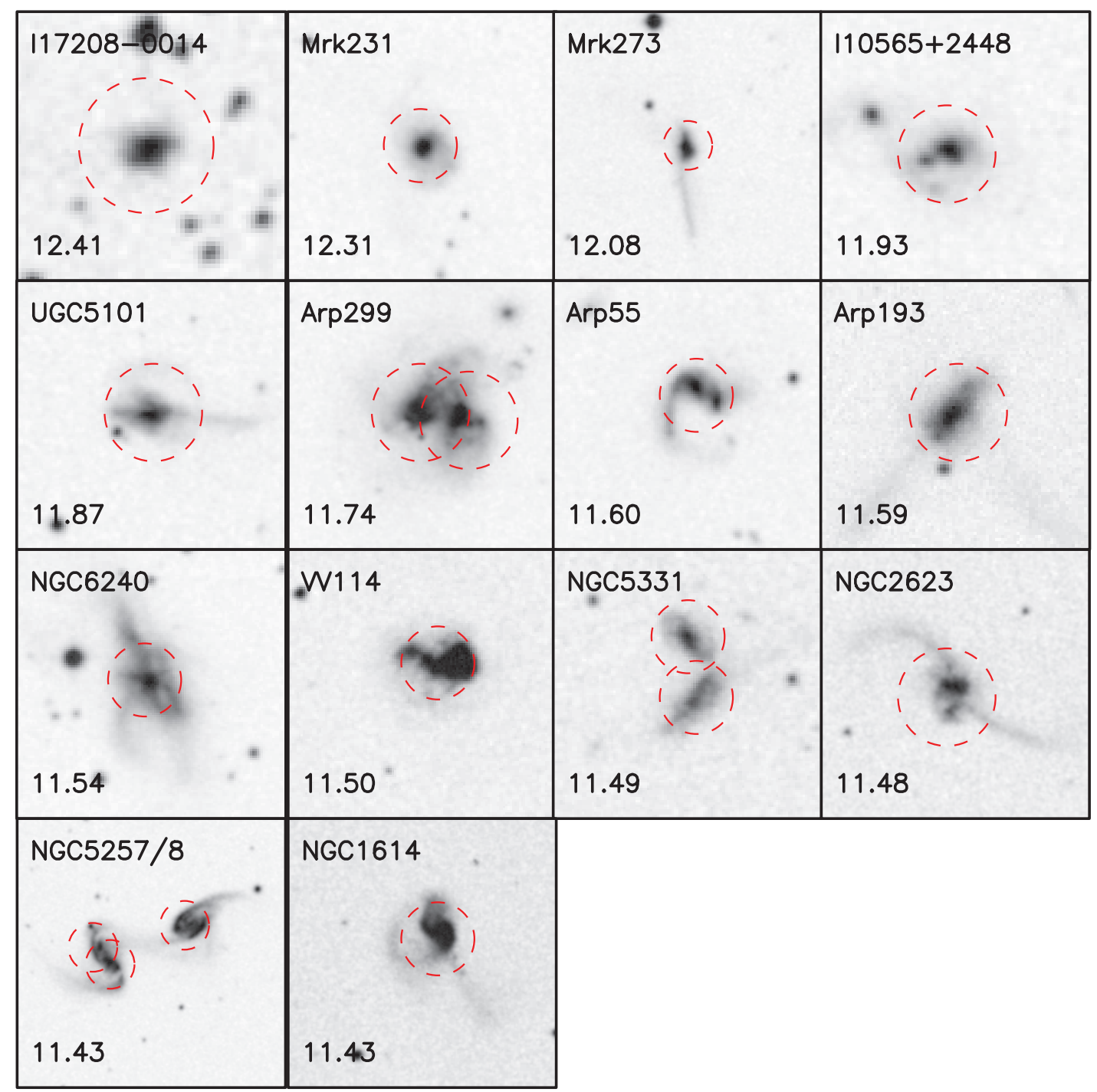

Fig. 1.-Optical images from the Second Digital Sky Survey for the 14 galaxies in our sample with the approximate half-power beam-width field of view of the SMA at $345 \mathrm{GHz}$ overlaid. Each plot is labeled with $\log L_{\mathrm{FIR}} / L_{\odot}$. In this figure and in the tables, the galaxies are presented in order of decreasing $L_{\mathrm{FIR}}$. 
TABLE 2

Observational Properties of the Survey

\begin{tabular}{|c|c|c|c|c|c|c|c|c|}
\hline \multirow[b]{2}{*}{ GALAXY } & \multicolumn{4}{|c|}{ CO $J=3-2$ Observations } & \multicolumn{4}{|c|}{ CO $J=2-1$ OBSERVATIONS } \\
\hline & Date Observed & $\begin{array}{c}\text { Number of } \\
\text { Antennas }\end{array}$ & $\begin{array}{l}\text { Sensitivity }^{\mathrm{a}} \\
{[\mathrm{mJy}(\mathrm{K})]}\end{array}$ & Time & Date Observed & $\begin{array}{c}\text { Number of } \\
\text { Antennas }\end{array}$ & $\begin{array}{l}\text { Sensitivity }^{\mathrm{a}} \\
{[\mathrm{mJy}(\mathrm{K})]}\end{array}$ & $\begin{array}{l}\text { Time } \\
\text { (hr) }\end{array}$ \\
\hline IRAS $17208-0014 \ldots \ldots \ldots \ldots$ & 2005 Aug 18, May 16 & 6 & $86(1.01)$ & 5.9 & $\ldots$ & $\ldots$ & $\ldots$ & $\ldots$ \\
\hline 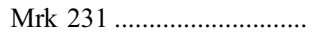 & 2006 Feb 05 & 8 & $33(0.55)$ & 3.3 & $\ldots$ & $\ldots$ & $\ldots$ & $\ldots$ \\
\hline 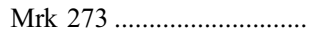 & 2006 Feb 04 & 7 & $45(0.67)$ & 3.8 & $\ldots$ & $\ldots$ & $\ldots$ & $\ldots$ \\
\hline IRAS $10565+2448 \ldots \ldots \ldots \ldots$ & 2006 Feb 02 & 7 & $25(0.46)$ & 3.7 & 2006 Feb 06 & 7 & $15(0.36)$ & 4.8 \\
\hline 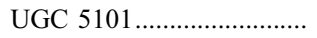 & 2006 Jan 30 & 6 & $32(0.56)$ & 5.6 & 2006 Feb 07 & 7 & $18(0.39)$ & 5.0 \\
\hline 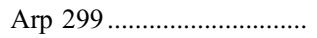 & 2006 Apr 10 & 7 & $37(0.087)$ & 5.6 & 2007 Mar 27 & 8 & $20(0.086)$ & 6.6 \\
\hline 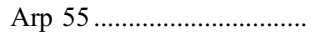 & 2006 Jan 29 & 6 & $40(0.74)$ & 4.3 & 2006 Feb 11 & 7 & $18(0.44)$ & 6.3 \\
\hline 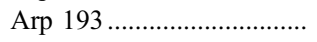 & 2006 Apr 16 & 8 & $38(0.093)$ & 3.6 & $\ldots$ & $\ldots$ & $\ldots$ & $\ldots$ \\
\hline 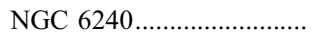 & 2005 Oct 09 , Oct 14 & 7 & $44(0.24)$ & 9.9 & $\ldots$ & $\ldots$ & $\ldots$ & $\ldots$ \\
\hline 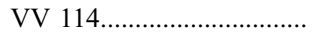 & 2005 Nov 13 & 7 & $31(0.057)$ & 4.4 & 2005 Nov 15, Nov 25 & 7 & $15(0.029)$ & 7.0 \\
\hline 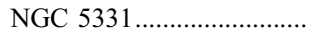 & 2007 Mar 25 & 8 & $50(0.23)$ & 6.3 & 2006 Apr 22 & 8 & $21(0.047)$ & 5.0 \\
\hline 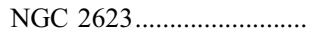 & 2007 Jan 16 & 8 & $12(0.028)$ & 6.7 & 2006 Apr 12, Apr 13, Mar 13 & 6,8 & $20(0.40)$ & 13.0 \\
\hline 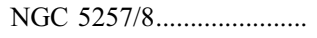 & $\ldots$ & $\ldots$ & $\ldots$ & $\ldots$ & 2006 Mar 18 & 8 & $51(0.12)$ & 4.8 \\
\hline 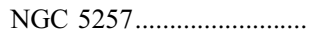 & 2006 Apr 11,2007 May 02 & 7,6 & $127(0.12)$ & 3.0 & $\cdots$ & $\cdots$ & $\cdots$ & $\cdots$ \\
\hline 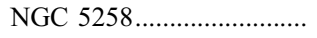 & 2006 Apr 11,2007 May 02 & 7,6 & $165(0.16)$ & 3.0 & $\ldots$ & $\ldots$ & $\ldots$ & $\ldots$ \\
\hline 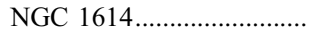 & 2005 Nov 14 & 7 & $41(0.079)$ & 5.1 & 2005 Nov 12 & 7 & $14(0.027)$ & 5.5 \\
\hline
\end{tabular}

${ }^{\text {a }}$ Noise level measured from the dirty map using line-free channels with $20 \mathrm{~km} \mathrm{~s}^{-1}$ resolution.

galaxies (29\% with $\log L_{\mathrm{FIR}}>11.9$ compared to $13 \%$ for the full sample), and has a mean far-infrared luminosity that is $35 \%$ larger $\left(0.13\right.$ in $\left.\log L_{\mathrm{FIR}}\right)$ than the full sample. Thus, the sample of galaxies discussed in this paper is slightly biased toward more luminous galaxies than the full luminosity and distance-limited sample.

\subsection{Data from the Submillimeter Array}

Observations with the Submillimeter Array (SMA: Ho et al. 2004) were obtained between 2005 May 16 and 2007 May 2. The correlator was configured to have a spectral resolution of 0.8125 $\mathrm{MHz}$ (typically $\sim 0.7 \mathrm{~km} \mathrm{~s}^{-1}$ for CO $J=3-2$ and $\sim 1.1 \mathrm{~km} \mathrm{~s}^{-1}$ for $\mathrm{CO} J=2-1$ ) and a bandwidth of $2 \mathrm{GHz}$, which was covered by 24 "chunks" overlapping slightly in frequency in each of the upper and lower sidebands. Each galaxy was observed with the CO $J=3-2$ transition in the lower sideband of the receiver and continuum in the upper sideband $10 \mathrm{GHz}$ away. The $\mathrm{HCO}^{+} J=$ 4-3 line lies in the high-frequency end of the upper sideband window and was detected in seven of the galaxies in our sample. In addition, nine galaxies were observed in the $\mathrm{CO}$ and ${ }^{13} \mathrm{CO} J=$ $2-1$ lines and in $1.3 \mathrm{~mm}$ continuum in a single tuning. It was not possible to also include the $\mathrm{C}^{18} \mathrm{O} J=2-1$ line in our passband due to the broad lines of these galaxies.

Each galaxy was observed in a single configuration of the SMA, either the extended array (unprojected baselines of 50-182 m) or the compact array (unprojected baselines of 16-69 m). The largest angular scale to which these data are sensitive, as calculated from the minimum $u v$-distance that the data sample well, ranges from $12^{\prime \prime}-16^{\prime \prime}\left(19^{\prime \prime}-25^{\prime \prime}\right)$ for the compact array CO $J=3-2(J=$ $2-1)$ data to $7^{\prime \prime}-10^{\prime \prime}\left(10^{\prime \prime}-15^{\prime \prime}\right)$ for the extended array $\mathrm{CO} J=$ $3-2(J=2-1)$ data.

The configuration used in each case was chosen to yield a spatial resolution of $0.7-1 \mathrm{kpc}$ in the $\mathrm{CO} J=3-2$ line; the typical angular resolution at this wavelength is $2.5^{\prime \prime}$ for the compact configuration and $0.9^{\prime \prime}$ for the extended configuration. The exceptions were NGC 2623 in CO $J=2-1$ and NGC 6240 and IRAS $17208-0014$ in CO $J=3-2$, which were observed in both the extended and compact arrays, and NGC 5257/8, which was observed in both the compact and subcompact arrays. For a given galaxy, the same configuration was used for both the $\mathrm{CO} J=2-1$ and the $\operatorname{CO} J=3-2$ observations. The field of view of the SMA is $\sim 60^{\prime \prime}$ for the $\mathrm{CO} J=2-1$ data and $\sim 40^{\prime \prime}$ for the $\mathrm{CO} J=3-2$ data. Three of the galaxies have sufficiently extended $\mathrm{CO}$ emission that a small mosaic of two (Arp 299, NGC 5331) or three (NGC 5257/58) pointings was used.

The data for each line-configuration combination in each galaxy were obtained in a single night's observing except for NGC 2623 (CO $J=2-1$ extended array data), NGC 5257/8 (CO $J=$ 3-2 data), and VV 114 (CO $J=2-1$ data). Between six and eight antennas were used in the observations, and the total onsource integration time ranged from 3.0 to $13.0 \mathrm{hr}$. The typical double sideband system temperatures at transit ranged from 240 to $800 \mathrm{~K}$ for the $\mathrm{CO} J=3-2$ data, except for IRAS $17208-$ 0014 , for which the values ranged from 650 to $1900 \mathrm{~K}$, and from 140 to $550 \mathrm{~K}$ for the $\mathrm{CO} J=2-1$ data. The observing dates, onsource integration times, and sensitivities obtained with robust weighting are given in Table 2.

The initial data calibration was done using the MIR software package. Observations of Uranus, Neptune, Ganymede, or Callisto were used to determine the current flux of the gain calibrator, which was a nearby quasar with a flux of at least $0.6 \mathrm{Jy}$. The amplitude and phase gain variations with time were calibrated using this gain calibrator. Antenna-based gain solutions were used throughout except for the extended array observations of NGC 2623 which required baseline-based gain solutions. Bandpass calibration was determined using a strong quasar such as 3C 273, 3C 279, or $3 \mathrm{C} 111$. We estimate the absolute flux calibration accuracy of these data to be $20 \%$. However, some of the galaxies are at redshifts such that ozone lines affect the atmospheric transmission in the $\mathrm{CO}$ $J=3-2$ line (Arp 55, UGC 5101) or $880 \mu$ m continuum (Arp 299, Mrk 273, and, to a lesser extent, Arp 55, Mrk 231, NGC 5331, and UGC 5101, for which only $200 \mathrm{MHz}$ of the continuum bandwidth might be affected); thus, the absolute calibration for these galaxies is more uncertain. After calibration in MIR, the data were exported to MIRIAD format (Sault et al. 1995) for further editing and imaging.

The data were first flagged to remove the six beginning and ending channels of each of the 24 chunks of the correlator. The data were also flagged to remove high-amplitude data points, 
TABLE 3

InTERFerometric CO ANd Continuum Fluxes

\begin{tabular}{|c|c|c|c|c|c|c|c|c|c|c|c|c|}
\hline Galaxy & $\begin{array}{l}\mathrm{CO} J=3-2 \text { Flux } \\
\left(\mathrm{Jy} \mathrm{km} \mathrm{s}^{-1}\right)\end{array}$ & $\begin{array}{c}\text { Beam } \\
(\operatorname{arcsec})\end{array}$ & $\begin{array}{l}880 \mu \mathrm{m}^{\mathrm{a}} \\
(\mathrm{mJy})\end{array}$ & $\begin{array}{c}\mathrm{HCO}^{+} J=4-3 \text { Flux }^{\mathrm{b}} \\
\left(\mathrm{Jy} \mathrm{km} \mathrm{s}^{-1}\right)\end{array}$ & $\begin{array}{l}\mathrm{CO} J=2-1 \text { Flux } \\
\left(\text { Jy km s} ~^{-1}\right)\end{array}$ & $\begin{array}{c}{ }^{13} \mathrm{CO} J=2-1 \text { Flux }{ }^{\mathrm{a}} \\
\left(\text { Jy km s s }{ }^{-1}\right)\end{array}$ & $\begin{array}{l}1.4 \mathrm{~mm}^{\mathrm{a}} \\
(\mathrm{mJy})\end{array}$ & $\begin{array}{l}\text { Beam } \\
(\operatorname{arcsec})\end{array}$ & $\begin{array}{l}\mathrm{CO} J=1-0 \text { Flux } \\
\left(\mathrm{Jy} \mathrm{km} \mathrm{s}^{-1}\right)\end{array}$ & $\begin{array}{l}3 \mathrm{~mm} \\
(\mathrm{mJy})\end{array}$ & $\begin{array}{l}\text { Beam } \\
(\operatorname{arcsec})\end{array}$ & Refs. $^{c}$ \\
\hline IRAS $17208-0014 \ldots \ldots \ldots \ldots$ & $478 \pm 33$ & $1.0 \times 0.9$ & $48 \pm 10$ & & $355 \pm 7$ & $\cdots$ & $37 \pm 3$ & $1.0 \times 0.7$ & 132 & $<5$ & $5.1 \times 1.6$ & 1,10 \\
\hline Mrk 231 & $308 \pm 8$ & $0.9 \times 0.8$ & $80 \pm 4$ & $25 \pm 3$ & 280 & $\ldots$ & 36 & $0.7 \times 0.5$ & 97 & 63 & $1.3 \times 1.1$ & 1 \\
\hline Mrk 273 & $441 \pm 14$ & $0.9 \times 0.8$ & $56 \pm 5$ & & 231 & $\ldots$ & 8 & 0.6 & 78 & 11 & $1.4 \times 1.3$ & 1 \\
\hline IRAS $10565+2448 \ldots \ldots \ldots \ldots$ & $204 \pm 7$ & $0.9 \times 0.7$ & $15 \pm 3$ & $\ldots$ & $187 \pm 6$ & $<2$ & $6 \pm 1$ & $1.1 \times 1.0$ & 68 & $<2$ & $2.3 \times 1.4$ & 1 \\
\hline UGC $5101 \ldots \ldots \ldots \ldots \ldots \ldots$ & $209 \pm 10$ & $1.0 \times 0.7$ & $37 \pm 9$ & & $237 \pm 10$ & $<4$ & $12 \pm 2$ & $1.2 \times 0.9$ & 50 & & $2.1 \times 1.6$ & 2 \\
\hline Arp 299 & $2582 \pm 34$ & $2.3 \times 1.9$ & $101 \pm 7$ & $\geq 67 \pm 5$ & $1976 \pm 16$ & $38.8 \pm 3.4$ & $46 \pm 6$ & $3.1 \times 1.8$ & 397 & $31 \pm 3$ & $2.5 \times 2.2$ & 3 \\
\hline IC 694 & $\geq 1610 \pm 27$ & “ & $81 \pm 5$ & $\geq 50 \pm 4$ & $1168 \pm 13$ & $17.5 \pm 2.7$ & $40 \pm 6$ & “ & 242 & $17 \pm 2$ & “ & 3 \\
\hline NGC $3690 \ldots \ldots$ & $\geq 596 \pm 17$ & “ & $20 \pm 5$ & $\geq 17 \pm 3$ & $355 \pm 8$ & $3.5 \pm 1.1$ & $<4$ & “ & 53 & $5 \pm 2$ & “ & 3 \\
\hline Arp 55 & $146 \pm 12$ & $0.9 \times 0.8$ & $26 \pm 7$ & $\ldots$ & $163 \pm 9$ & $<6$ & $<2$ & $1.1 \times 0.9$ & 83 & $\ldots$ & $9 \times 7$ & 4 \\
\hline Arp 55(NE) & $94 \pm 9$ & $0.9 \times 0.8$ & $26 \pm 7$ & & $110 \pm 8$ & $<3$ & $<2$ & “ & .. & & “ & 4 \\
\hline 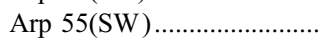 & $52 \pm 8$ & $0.9 \times 0.8$ & $<8$ & $\ldots$ & $53 \pm 4$ & $<3$ & $<2$ & “ & $\ldots$ & $\ldots$ & “ & 4 \\
\hline Arp 193 & $886 \pm 15$ & $2.2 \times 2.0$ & $39 \pm 4$ & $\geq 21 \pm 5$ & 450 & $\ldots$ & 10 & $0.6 \times 0.4$ & 161 & $<5$ & $1.3 \times 0.9$ & 1 \\
\hline NGC $6240 \ldots \ldots$ & $2428 \pm 52$ & $1.6 \times 1.3$ & $33 \pm 13$ & $\geq 166 \pm 12$ & 1220 & $\ldots$ & $5.9 \pm 0.3$ & $0.9 \times 0.5$ & 324 & $\ldots$ & $4.9 \times 4.1$ & 7,9 \\
\hline NGC 6240 (central) ............... & $2318 \pm 48$ & $1.6 \times 1.3$ & $33 \pm 13$ & $\geq 166 \pm 12$ & 1220 & $\ldots$ & $5.9 \pm 0.3$ & $0.9 \times 0.5$ & 324 & $\ldots$ & $4.9 \times 4.1$ & 7,9 \\
\hline NGC 6240(WC) ................. & $110 \pm 19$ & $1.6 \times 1.3$ & $<16$ & & $\ldots$ & $\ldots$ & $\ldots$ & & & & & 7,9 \\
\hline VV $114 \ldots \ldots \ldots \ldots$ & $1530 \pm 16$ & $2.8 \times 2.0$ & $26 \pm 6$ & $\geq 17 \pm 2$ & $1109 \pm 8$ & $10.6 \pm 2.6^{\mathrm{d}}$ & $11 \pm 2$ & $4.1 \times 3.0$ & 674 & $<16$ & $4.4 \times 3.1$ & 5 \\
\hline 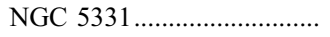 & $478 \pm 26$ & $2.3 \times 1.0$ & $27 \pm 6$ & & $333 \pm 11$ & $8.8 \pm 2.4$ & $8 \pm 2$ & $3.5 \times 3.1$ & 167 & & $5.4 \times 4.4$ & 6 \\
\hline 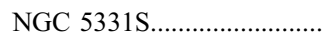 & $401 \pm 24$ & “ & $27 \pm 6$ & $\ldots$ & $250 \pm 7$ & $8.8 \pm 2.4$ & $8 \pm 2$ & “ & 150 & $\ldots$ & “ & 6 \\
\hline NGC 5331N & $77 \pm 10$ & “ & $<12$ & $\ldots$ & $83 \pm 9$ & $<4$ & $<4$ & “ & 17 & $\ldots$ & “ & 6 \\
\hline NGC $2623 \ldots \ldots \ldots$ & $607 \pm 5$ & $2.2 \times 2.0$ & $50 \pm 2$ & $27 \pm 2$ & $267 \pm 8$ & $<3$ & $17 \pm 3$ & $1.2 \times 1.0$ & 153 & $\ldots$ & $3.5 \times 2.4$ & 7 \\
\hline NGC $5257 / 8 \ldots \ldots \ldots \ldots \ldots$ & $1140 \pm 70$ & $3.8 \times 3.0$ & $104 \pm 21$ & $\ldots$ & $344 \pm 51$ & $<20$ & $<16$ & $3.5 \times 2.8$ & 387 & $\cdots$ & $6.2 \times 3.7$ & 6 \\
\hline NGC $5257 \ldots \ldots \ldots \ldots \ldots$ & $286 \pm 47$ & “ & $<26$ & $\ldots$ & $109 \pm 24$ & $<10$ & $<8$ & “ & 137 & $\ldots$ & “ & 6 \\
\hline NGC 5258........................... & $854 \pm 52$ & “ & $104 \pm 21^{\mathrm{e}}$ & $\ldots$ & $235 \pm 45$ & $<10$ & $<8$ & “ & 250 & $\ldots$ & “ & 6 \\
\hline 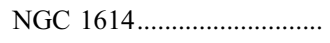 & $674 \pm 14$ & $2.6 \times 2.1$ & $27 \pm 7$ & $\geq 14 \pm 3$ & $670 \pm 7$ & $17.3 \pm 2.7$ & $21 \pm 3$ & $3.7 \times 3.3$ & 215 & & $4 \times 6$ & 8 \\
\hline
\end{tabular}

${ }^{\text {a }}$ Upper limits are $2 \sigma$ in a single beam (per galaxy component, if applicable).

Lower limits indicated that the $\mathrm{HCO}^{+} J=4-3$ flux of the galaxy is uncertain because the full width of the line may not be contained within the spectrometer; see text.

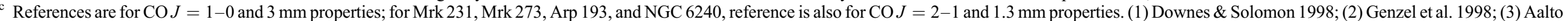
et al. 1997; (4) Sanders et al. 1988b; (5) Yun et al. 1994; (6) Iono et al. 2005; (7) Bryant \& Scoville 1999; (8) Scoville et al. 1989; (9) Tacconi et al. 1999; (10) L. Tacconi \& A. Baker 2007, private communication.

${ }^{\mathrm{d}}$ Only the eastern peak of VV 114 is detected in the ${ }^{13} \mathrm{CO} J=2-1$ line.

e NGC 5258 contains an off-nuclear continuum source whose flux is quoted here. The nucleus is undetected at $<30 \mathrm{mJy}(2 \sigma)$. 

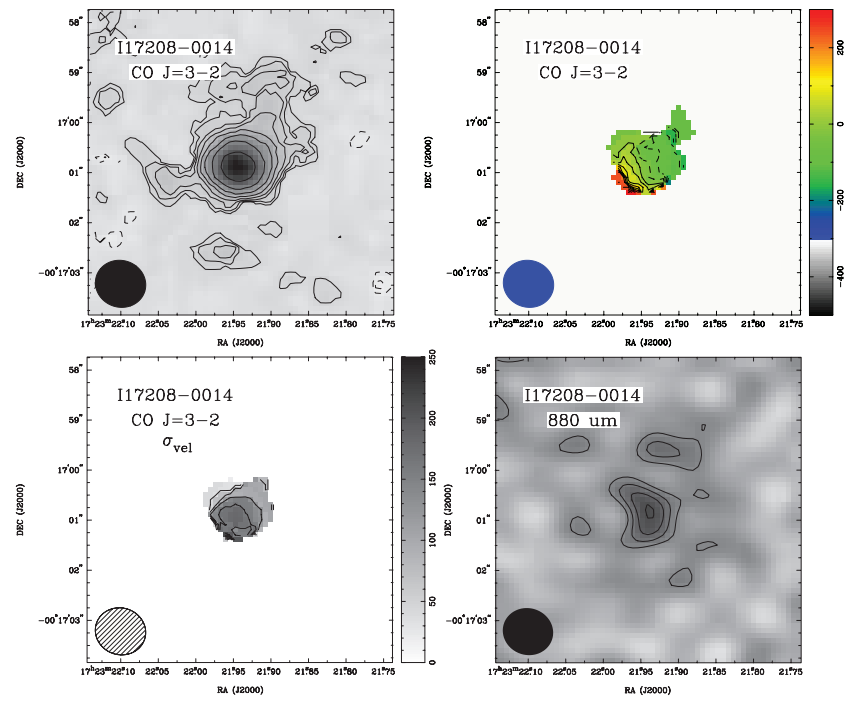

FIG. 2.-IRAS $17208-0014$ CO $J=3-2$ and $880 \mu \mathrm{m}$ continuum maps. See $\S 2$ for additional details of the data processing. Top left: $\mathrm{CO} J=3-2$ moment 0 map. Lowest contour is $2 \sigma=11.4 \mathrm{Jy}_{\text {beam }}^{-1} \mathrm{~km} \mathrm{~s}^{-1}$ and contours increase by factors of 1.5. Negative contours are shown as dashed lines. The synthesized beam is shown in the bottom left corner of this and subsequent panels. Top right: $\mathrm{CO}$ $J=3-2$ moment 1 map. Contours are $40 \mathrm{~km} \mathrm{~s}^{-1} \times(-5,-4,-3,-2,-1,0,1,2$, $3,4,5,6,7)$ relative to $c z$ with negative contours shown as dashed lines. Because of the high noise level, for only this galaxy the moment 1 and moment 2 maps were made using $40 \mathrm{~km} \mathrm{~s}^{-1}$ channel maps with a $4 \sigma$ flux cutoff. Note that the negative side of the rotation curve peaks at $-160 \mathrm{~km} \mathrm{~s}^{-1}$ and then drops to $-80 \mathrm{~km} \mathrm{~s}^{-1}$ at the extreme northwest end of the emission. Bottom right: $\mathrm{CO} J=3-2$ moment 2 map. Contours are $40 \mathrm{~km} \mathrm{~s}^{-1} \times(1,2,3,4,5)$. This figure plots velocity dispersion, $\sigma_{v}$ which for a Gaussian line relates to the full-width half-maximum velocity via $V_{\mathrm{FWHM}}=2.355 \sigma_{v}$. Bottom right: Uncleaned $880 \mu \mathrm{m}$ map. Lowest contour is $2 \sigma=8.2 \mathrm{mJy}$ and contours increase in steps of $1 \sigma$. Negative contours are not shown.

with typically $<1 \%$ of the data removed in this step. For a few data sets, additional flagging was used to remove data at the beginning or end of the track when deteriorating weather conditions or high system temperatures at lower elevations had caused a larger amplitude scatter. Data cubes were made using velocity resolutions of 10,20 , and $40 \mathrm{~km} \mathrm{~s}^{-1}$ for the $\mathrm{CO} J=3-2$ and $J=2-1$ lines, 20, 40, and $100 \mathrm{~km} \mathrm{~s}^{-1}$ for the ${ }^{13} \mathrm{CO} J=2-1$ line, and 40 and $100 \mathrm{~km} \mathrm{~s}^{-1}$ for the $\mathrm{HCO}^{+} J=4-3$ line. For the ${ }^{13} \mathrm{CO} J=2-1$ and $\mathrm{HCO}^{+}$data sets, where significant continuum emission was detected (see below), the continuum emission was subtracted in the $u v$-plane using line-free channels before imaging. All data cubes were inverted with weighting by the system temperature and moderate robustness, which gives the optimal trade off between sensitivity and resolution.

Each data cube was cleaned down to 2 times the rms noise, except for NGC 6240, which was cleaned down to the rms noise. For most sources, the beam was sufficiently clean and the emission sufficiently compact that cleaning the inner quarter of the image produced good results. However, four sources located near the equator (IRAS 17208-0014, NGC 5331, NGC 5257, and NGC 6240) had large sidelobes and/or extended emission and were cleaned using regions chosen by inspecting the dirty maps to isolate the emission from the galaxy; the same fixed regions were used for all velocity channels. The final data cubes were corrected for the attenuation of the SMA primary beam or the analogous response for the combined mosaic before we measured the integrated source fluxes.

Continuum maps at $1.3 \mathrm{~mm}$ and $880 \mu \mathrm{m}$ were made by imaging the individual line-free regions of the spectra; there were four such regions at $1.3 \mathrm{~mm}$ (one on either side of each of the $\mathrm{CO}$

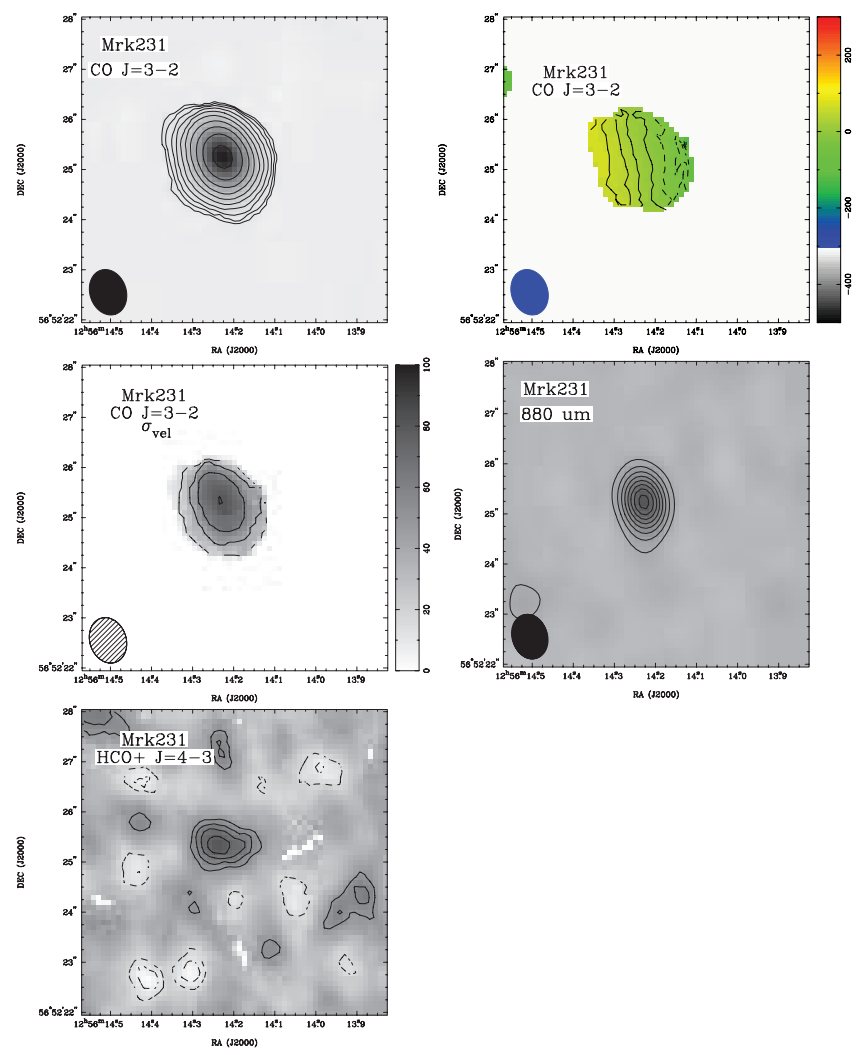

FIG. 3.-Mrk $231 \mathrm{CO} J=3-2$ and $880 \mu$ m continuum maps. Notation as in Fig. 2. Top left: $\mathrm{CO} J=3-2$ moment 0 map. Lowest contour is $2 \sigma=5.2 \mathrm{Jy}_{\text {beam }}{ }^{-1} \mathrm{~km} \mathrm{~s}^{-1}$, and contours increase by factors of 1.5. Top right: $\mathrm{CO} J=3-2$ moment 1 map. Contours are $20 \mathrm{~km} \mathrm{~s}^{-1} \times(-6,-5,-4,-3,-2,-1,0,1,2,3,4)$ relative to $c z$. Middle left: $\mathrm{CO} J=3-2$ moment 2 map. Contours are $20 \mathrm{~km} \mathrm{~s}^{-1} \times(1,2,3,4)$. Middle right: $880 \mu \mathrm{m}$ map. Lowest contour is $2 \sigma=8.2 \mathrm{mJy}$ and contours increase in steps of $2 \sigma$. Because of the strength of the central source, the cleaned continuum image is shown for this galaxy only. Bottom left: Uncleaned $\mathrm{HCO}^{+}$ $J=4-3$ moment 0 map. Lowest contour is $\pm 2 \sigma=7.92 \mathrm{Jy} \mathrm{beam}^{-1} \mathrm{~km} \mathrm{~s}^{-1}$, and contours increase in steps of $1 \sigma$. This image has been corrected for continuum emission by subtracting the $880 \mu \mathrm{m}$ continuum in the $u v$-plane before imaging.

and ${ }^{13} \mathrm{CO} J=2-1$ lines) and three regions at $880 \mu \mathrm{m}$ (one on either side of the $\mathrm{CO} J=3-2$ line and a single broad region in the upper sideband that excluded the region of the spectrum containing possible emission from the $\mathrm{HCO}^{+} J=4-3$ line). These continuum images were then averaged together, weighting by the inverse of the product of the bandwidth of each image and the square of its noise, to obtain a single continuum image with the best sensitivity. The continuum images were not cleaned, as the signal-to-noise ratio was low and the source(s) were generally compact and well separated from any sidelobes of the main beam. The one exception is Mrk 231, where the central source was sufficiently strong that cleaning was useful.

Table 3 gives the continuum and integrated ${ }^{12} \mathrm{CO},{ }^{13} \mathrm{CO}$, and $\mathrm{HCO}^{+}$fluxes measured for the galaxies in our sample. The fluxes measured for the $\mathrm{CO} J=3-2$ line and $880 \mu \mathrm{m}$ continuum emission for NGC 6240 differ somewhat from the values given in Iono et al. (2007) due to the different weighting used. Previously published data for the CO $J=2-1$ and $J=1-0$ lines are also given where available. Figures $2-24$ show the moment 0,1 , and 2 images for the $\operatorname{CO} J=3-2$ and $\operatorname{CO} J=2-1$ data, the $880 \mu \mathrm{m}$ and $1.3 \mathrm{~mm}$ dirty maps where signal was detected from the galaxy at the $3 \sigma$ level or better, and the ${ }^{13} \mathrm{CO} J=2-1$ and $\mathrm{HCO}^{+}$ $J=4-3$ integrated intensity maps, where these lines were detected. Unless otherwise noted in the figure captions, the moment 0 maps have been made from the $40 \mathrm{~km} \mathrm{~s}^{-1}$ resolution data 

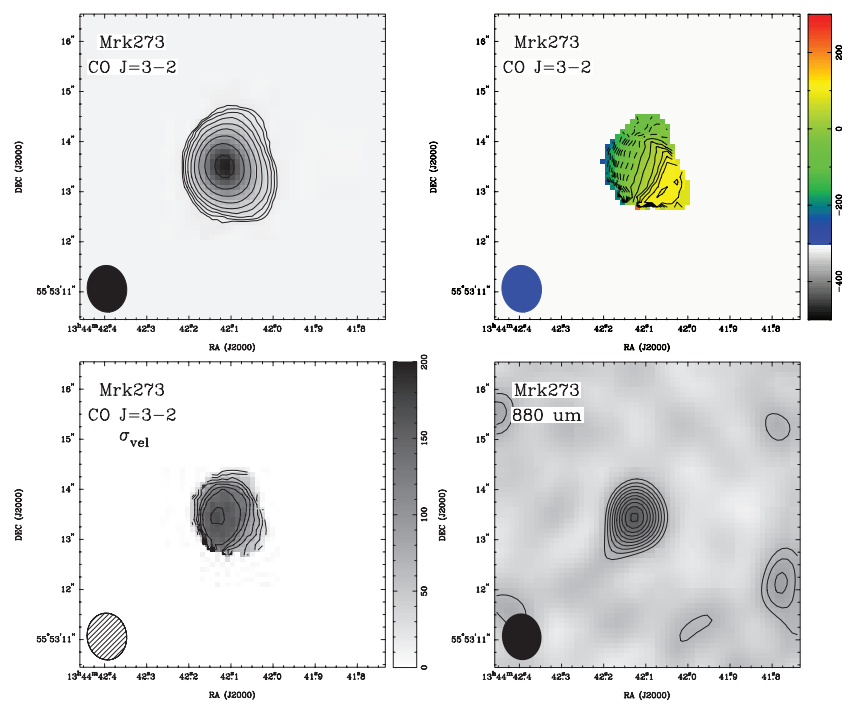

Fig. 4.-Mrk $273 \mathrm{CO} J=3-2$ and $880 \mu \mathrm{m}$ continuum maps. Notation as in Fig. 2. Top left: $\mathrm{CO} J=3-2$ moment 0 map. Lowest contour is $2 \sigma=5.2 \mathrm{Jy}_{\text {beam }}{ }^{-1}$ $\mathrm{km} \mathrm{s}^{-1}$, and contours increase by factors of 1.5. Top right: $\mathrm{CO} J=3-2$ moment 1 map. Contours are $20 \mathrm{~km} \mathrm{~s}^{-1} \times(-11,-10,-9,-8,-7,-6,-5,-4,-3,-2$, $-1,0,1,2,3,4,5,6)$ relative to $c z$. Bottom left: $\mathrm{CO} J=3-2$ moment 2 map. Contours are $20 \mathrm{~km} \mathrm{~s}^{-1} \times(1,2,3,4,5,6,7,8)$. Bottom right: Uncleaned $880 \mu \mathrm{m}$ map. Lowest contour is $2 \sigma=10.0 \mathrm{mJy}$, and contours increase in steps of $1 \sigma$. Residual sidelobes can be seen at the edges of this figure.

cube using only signal greater than $\pm 2 \sigma$, while the moment 1 and 2 maps have been made from the $20 \mathrm{~km} \mathrm{~s}^{-1}$ resolution data cube using only positive signal greater than $4 \sigma$. These images have not been corrected for the primary beam or mosaic response. Figures 25-29 show the $\mathrm{CO} J=3-2$ spectra, Figures $30-33$ show the $\mathrm{CO} J=2-1$ spectra, Figures 34 and 35 show the ${ }^{13} \mathrm{CO}$ $J=2-1$ spectra and Figure 36 shows the $\mathrm{HCO}^{+} J=4-3$ spectra. In all cases except $\mathrm{HCO}^{+}$, both the spectrum at the position of the emission peak and the spectrum integrated over the entire region with emission are shown.
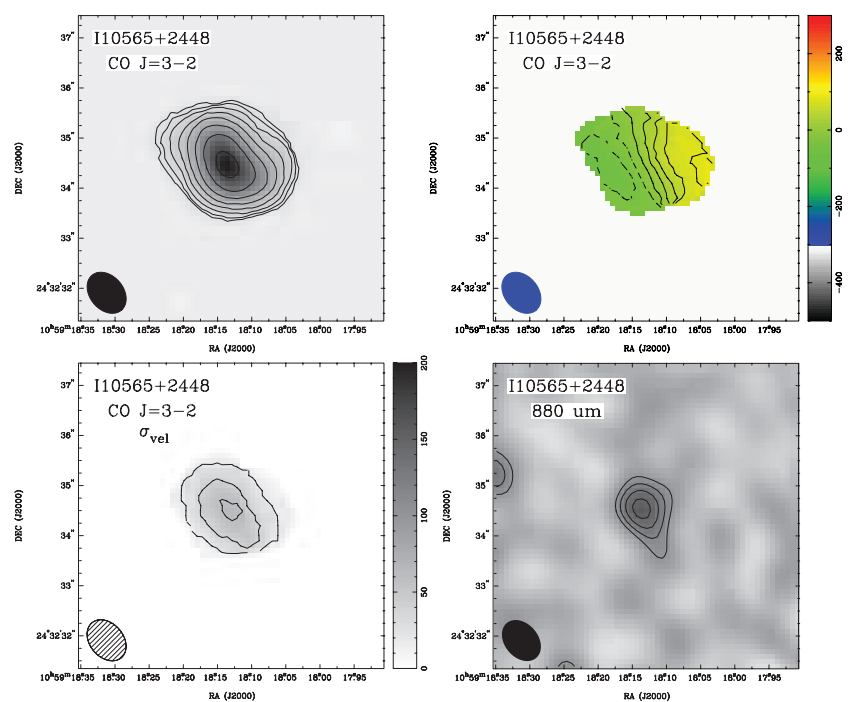

FIG. 5.-IRAS $10565 \mathrm{CO} J=3-2$ and $880 \mu$ m continuum maps. Notation as in Fig. 2. Top left: $\mathrm{CO} J=3-2$ moment 0 map. Lowest contour is $2 \sigma=4.3 \mathrm{Jy}_{\text {beam }}{ }^{-1}$ $\mathrm{km} \mathrm{s}^{-1}$, and contours increase by factors of 1.5. Top right: $\mathrm{CO} J=3-2$ moment 1 map. Contours are $20 \mathrm{~km} \mathrm{~s}^{-1} \times(-2,-1,0,1,2,3,4)$ relative to cz. Bottom left: CO $J=3-2$ moment 2 map. Contours are $20 \mathrm{~km} \mathrm{~s}^{-1} \times(1,2,3)$. Bottom right: Uncleaned $880 \mu \mathrm{m}$ map. Lowest contour is $2 \sigma=5.4 \mathrm{mJy}$, and contours increase in steps of $1 \sigma$. A residual sidelobe can be seen at the eastern edge of this figure.
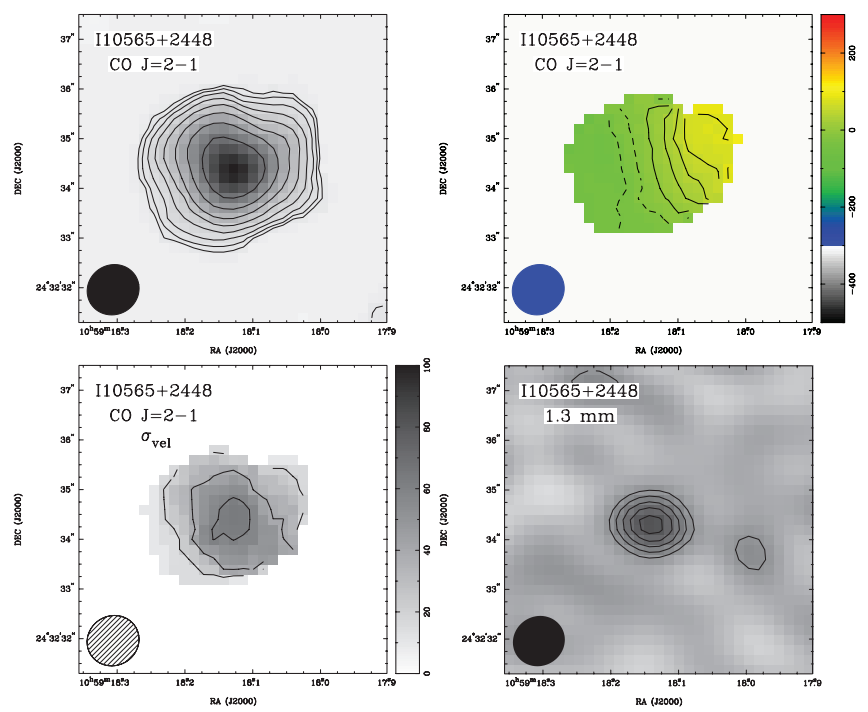

FIG. 6.-IRAS $10565 \mathrm{CO} J=2-1$ and $1.3 \mathrm{~mm}$ continuum maps. Notation as in Fig. 2. Top left: $\operatorname{CO} J=2-1$ moment 0 map. Lowest contour is $\pm 2 \sigma=$ $2.7 \mathrm{Jy}_{\text {beam }}{ }^{-1} \mathrm{~km} \mathrm{~s}^{-1}$, and contours increase by factors of 1.5 . Top right: $\mathrm{CO}$ $J=2-1$ moment 1 map. Contours are $20 \mathrm{~km} \mathrm{~s}^{-1} \times(-2,-1,0,1,2,3,4)$ relative to $c z$. Bottom left: $\mathrm{CO} J=2-1$ moment 2 map. Contours are $20 \mathrm{~km} \mathrm{~s}^{-1} \times$ $(1,2,3)$. Bottom right: Uncleaned $1.3 \mathrm{~mm}$ map. Lowest contour is $2 \sigma=2.0 \mathrm{mJy}$ and contours increase in steps of $1 \sigma$.

\subsubsection{Morphology and Kinematics of the CO Emission}

Figures 2-24 reveal a diversity of morphologies and kinematics for the molecular gas in our sample of galaxies. Nine of the galaxies show a predominantly compact morphology while the remaining five galaxies range from well-separated pairs of galaxies to highly complex morphologies. The galaxies with extended $\mathrm{CO}$ emission are all found in the lower luminosity half of our sample, although some low-luminosity galaxies also show a compact morphology. The source sizes derived from two-dimensional
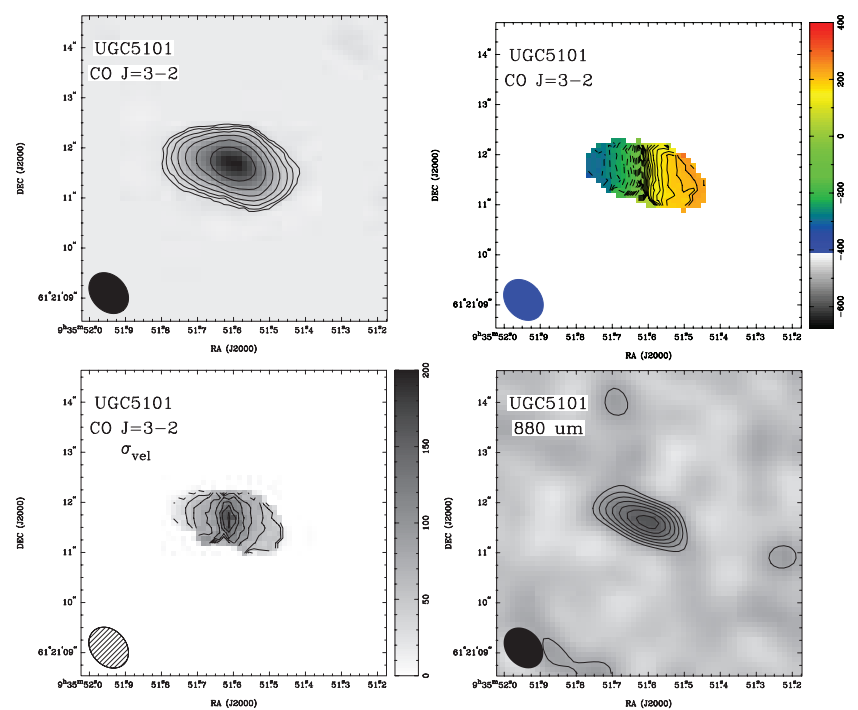

FIG. 7.- UGC $5101 \mathrm{CO} J=3-2$ and $880 \mu \mathrm{m}$ continuum maps. Notation as in Fig. 2. Top left: $\mathrm{CO} J=3-2$ moment 0 map. Lowest contour is $2 \sigma=7.6 \mathrm{Jy} \mathrm{beam}^{-1}$ $\mathrm{km} \mathrm{s}^{-1}$, and contours increase by factors of 1.5 . Top right: $\mathrm{CO} J=3-2$ moment 1 map. Contours are $20 \mathrm{~km} \mathrm{~s}^{-1} \times(-15,-14,-13, \ldots-2,-1,0,1,2, \ldots$, $10,11)$ relative to $c z$. Bottom left: $\operatorname{CO} J=3-2$ moment 2 map. Contours are $20 \mathrm{~km} \mathrm{~s}^{-1} \times(1,2,3,4,5,6,7,8)$. Bottom right: Uncleaned $880 \mu \mathrm{m}$ map. Lowest contour is $2 \sigma=5.4 \mathrm{mJy}$ and contours increase in steps of $1 \sigma$. Sidelobes can be seen at the edges of this figure. 

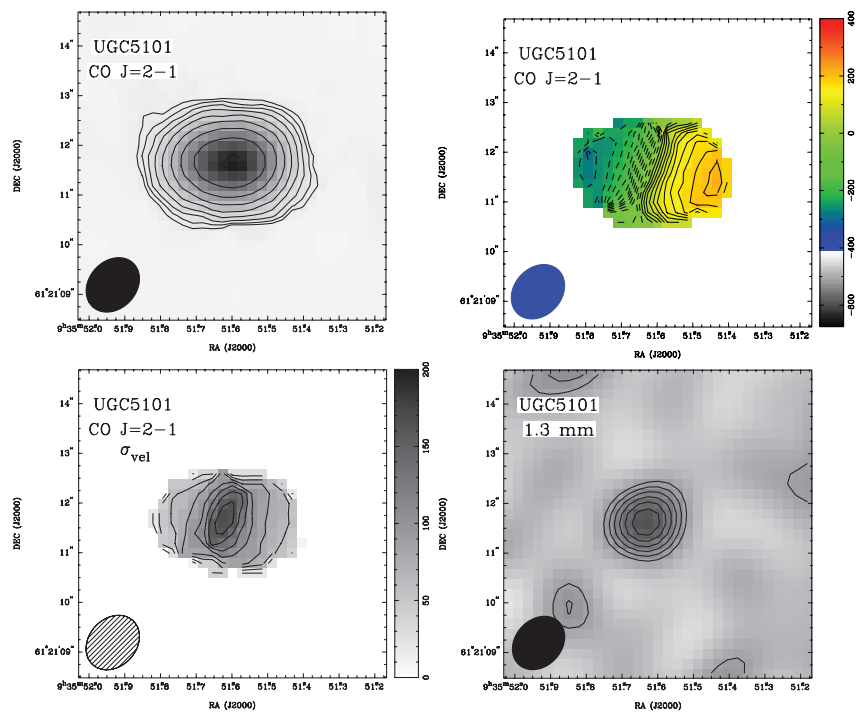

FIG. 8.-UGC $5101 \mathrm{CO} J=2-1$ and $1.3 \mathrm{~mm}$ continuum maps. Notation as in Fig. 2. Top left: $\mathrm{CO} J=2-1$ moment 0 map. Lowest contour is $\pm 2 \sigma=$ $4.6 \mathrm{Jy}_{\text {beam }}^{-1} \mathrm{~km} \mathrm{~s}^{-1}$, and contours increase by factors of 1.5. Top right: $\mathrm{CO}$ $J=2-1$ moment 1 map. Contours are $20 \mathrm{~km} \mathrm{~s}^{-1} \times(-13,-12,-11, \ldots-2$, $-1,0,1,2, \ldots, 8,9,10)$ relative to $c z$. Bottom left: $\mathrm{CO} J=2-1$ moment 2 map. Contours are $20 \mathrm{~km} \mathrm{~s}^{-1} \times(1,2,3,4,5,6,7,8)$. Bottom right: Uncleaned $1.3 \mathrm{~mm}$ map. Lowest contour is $2 \sigma=3.0 \mathrm{mJy}$ and contours increase in steps of $1 \sigma$.
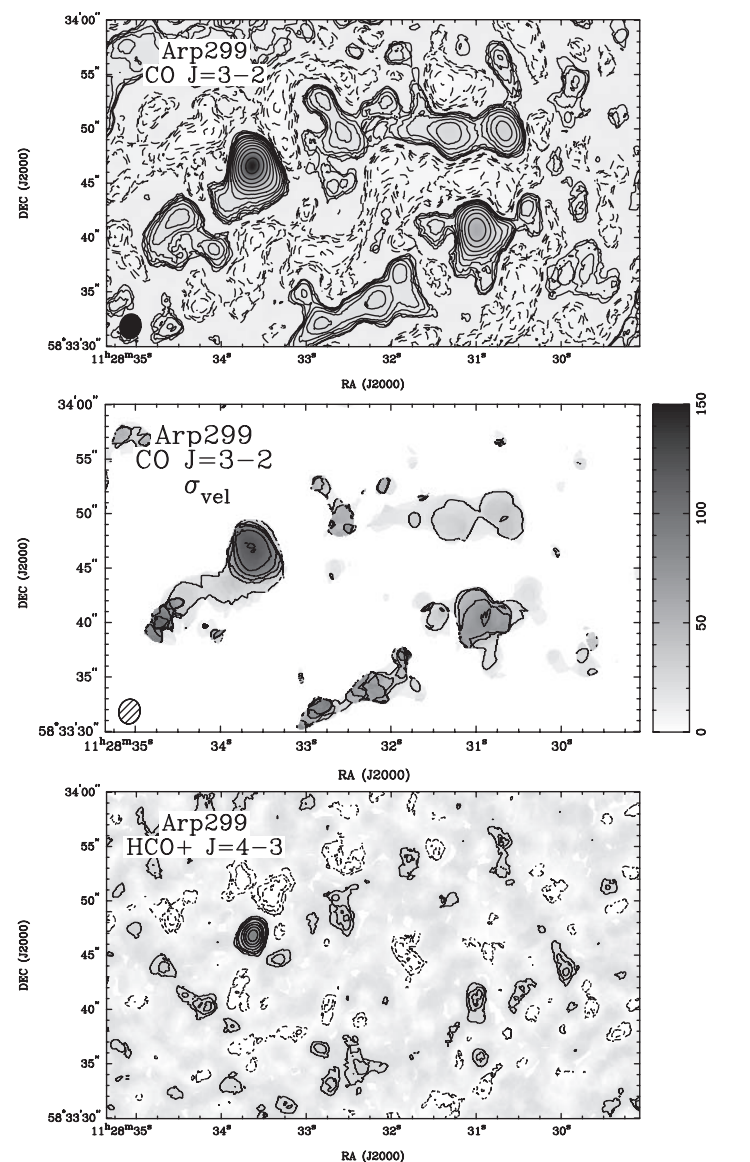

Gaussian fits to the $\mathrm{CO} J=3-2$ images and the line widths (full width at half-maximum) derived from the $\mathrm{CO} J=3-2$ spectra are tabulated in Iono et al. (2008). The distributions of the CO $J=3-2$ and $J=2-1$ emissions are qualitatively similar; we will discuss the line ratio distributions in more detail in a future paper.

The kinematics of the molecular gas are also quite varied. Integrating over the full emission region in each galaxy (Figs. 25$33)$, the line widths at half-maximum range from $200 \mathrm{~km} \mathrm{~s}^{-1}$ in Mrk 231 to $650 \mathrm{~km} \mathrm{~s}^{-1}$ in UGC 5101. Some of the line profiles are quite smooth and Gaussian (e.g., Mrk 231), while others have more of a double-peaked structure (e.g., UGC 5101). The maps of the velocity fields (Figs. 2-24) show many examples of smooth and regular rotation among the more compact galaxies, although some of the compact galaxies, such as Mrk 273 and NGC 6240, are significantly distorted. Two of the galaxies with extended $\mathrm{CO}$ emission, Arp 299 and VV 114 show very complex and distorted velocity fields. The maps of the velocity dispersions show that the peak velocity dispersion is commonly $80-100 \mathrm{~km} \mathrm{~s}^{-1}$. However, the peak velocity dispersions for IRAS 17208-0014, Mrk 273, UGC 5101, NGC 6240, and NGC 5331S are twice as large as the typical galaxy in our sample. The lower velocity dispersions seen for NGC 5257/8 are likely due to the lower signalto-noise of those maps; similarly, a lower velocity dispersion is seen for NGC 5331S in the CO $J=3-2$ line than in the CO $J=2-1$ line.

Our velocity dispersions are typically about $70 \%$ of the values measured from CO band-head emission in the near-infrared
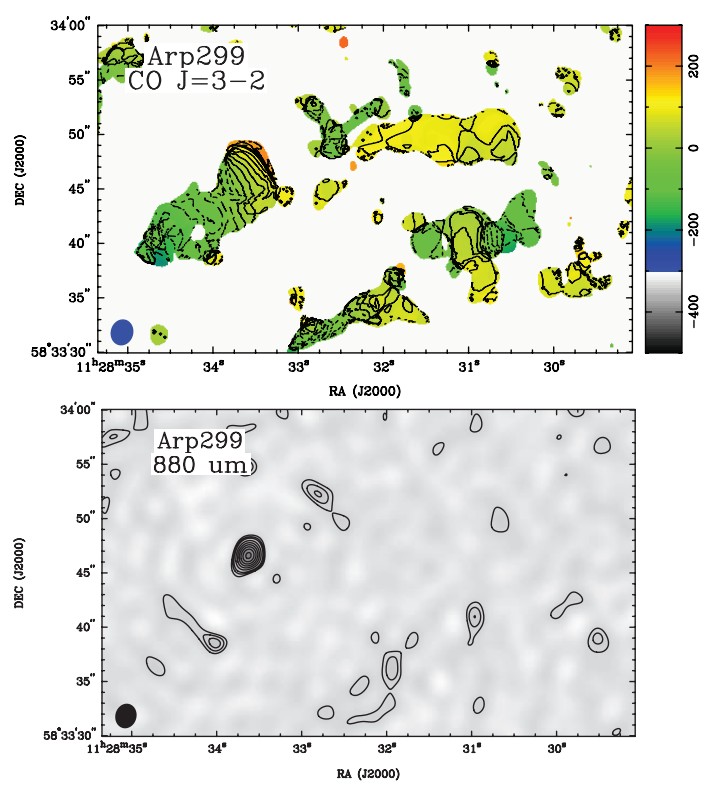

FIG. 9.-Arp $299 \mathrm{CO} J=3-2$ and $880 \mu \mathrm{m}$ continuum maps. Notation as in Figs. 2 and 3. Top left: CO J $=3-2$ moment 0 map. Lowest contour is $\pm 2 \sigma=8.7$ Jy beam ${ }^{-1}$

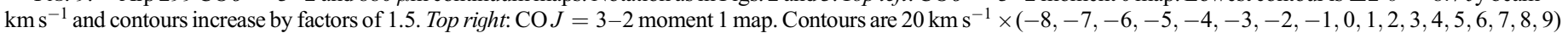

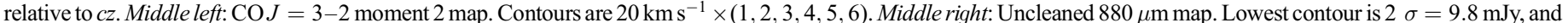

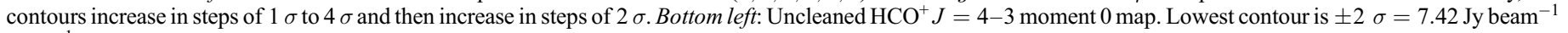
$\mathrm{km} \mathrm{s}^{-1}$, and contours increase by in steps of $1 \sigma$ to $4 \sigma$ and then by steps of $2 \sigma$. 

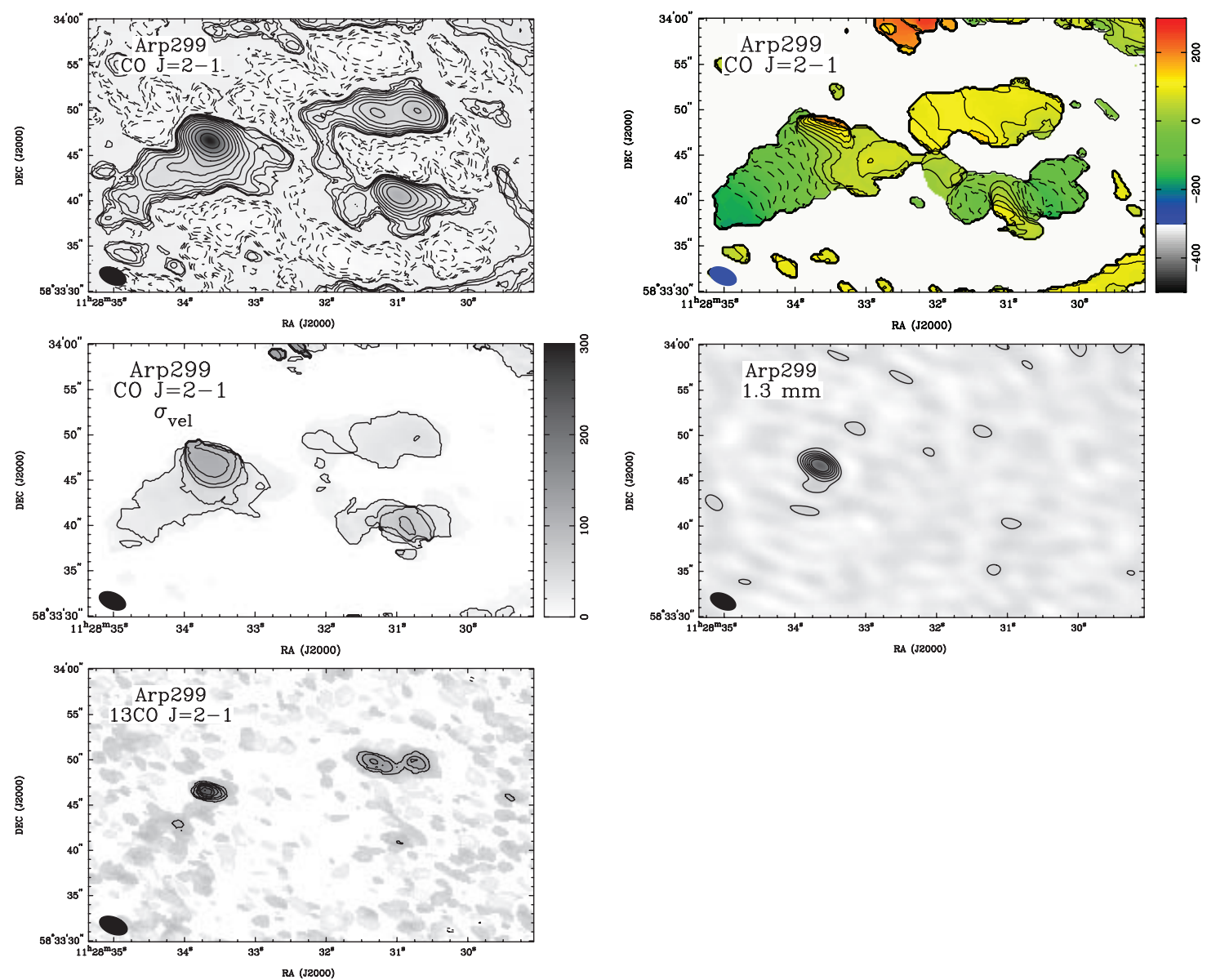

FIG. 10.-Arp $299 \mathrm{CO} J=2-1$ and $1.3 \mathrm{~mm}$ continuum maps. Notation as in Fig. 2. Top left: $\mathrm{CO} J=2-1$ moment 0 map. Lowest contour is $\pm 2 \sigma=4.6 \mathrm{Jy}$ beam ${ }^{-1}$ $\mathrm{km} \mathrm{s}^{-1}$, and contours increase by factors of 1.5. Top right: $\mathrm{CO} J=2-1$ moment 1 map. Contours are $20 \mathrm{~km} \mathrm{~s}^{-1} \times(-9,-8,-7,-6,-5,-4,-3,-2,-1,0,1,2,3,4,5$, $6,7,8,9)$ relative to $c z$. Middle left: $\mathrm{CO} J=2-1$ moment 2 map. Contours are $20 \mathrm{~km} \mathrm{~s}^{-1} \times(1,2,3,4,5)$. Middle right: Uncleaned $1.3 \mathrm{~mm}$ map. Lowest contour is $2 \sigma=5.4 \mathrm{mJy}$, and contours increase in steps of $2 \sigma$. Bottom left: ${ }^{13} \mathrm{CO} J=2-1$ moment 0 map. Lowest contour is $\pm 2 \sigma=3.9 \mathrm{Jy} \mathrm{beam}^{-1} \mathrm{~km} \mathrm{~s}^{-1}$ and contours increase in steps of $1 \sigma$. This image has been corrected for continuum emission by subtracting the $1.3 \mathrm{~mm}$ continuum in the $u v$-plane before imaging.
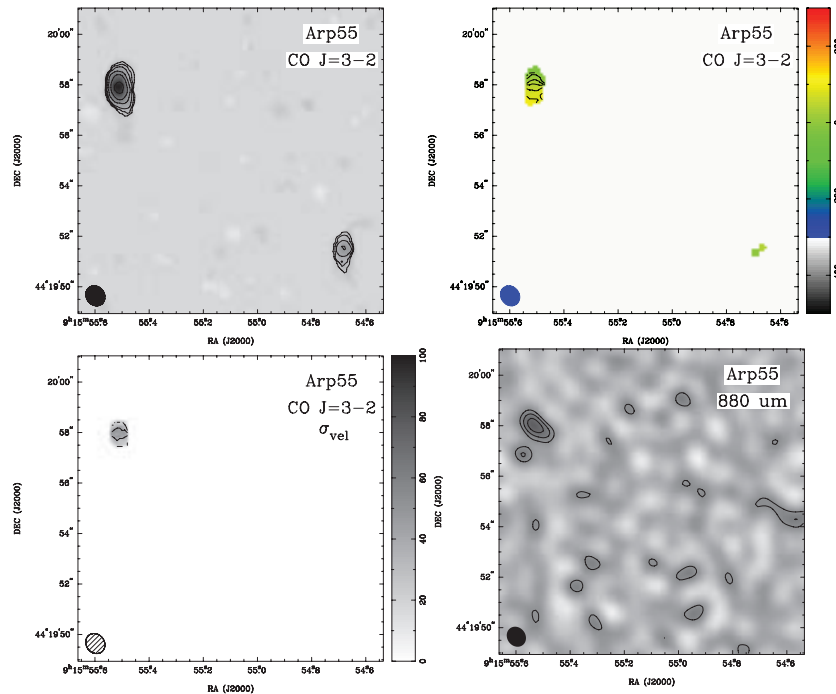

FIg. 11.- Arp $55 \mathrm{CO} J=3-2$ and $880 \mu$ m continuum maps. Notation as in Fig. 2. Top left: $\mathrm{CO} J=3-2$ moment 0 map. Lowest contour is $2 \sigma=6.0 \mathrm{Jy} \mathrm{beam}^{-1} \mathrm{~km} \mathrm{~s}^{-1}$, and contours increase by factors of 1.5 . Top right: $\mathrm{CO} J=3-2$ moment 1 map. Contours are $20 \mathrm{~km} \mathrm{~s}^{-1} \times(-1,0,1,2,3,4)$ relative to $c z$. Bottom left: $\mathrm{CO} J=$ $3-2$ moment 2 map. Contours are $20 \mathrm{~km} \mathrm{~s}^{-1} \times(1,2)$. Bottom right: Uncleaned $880 \mu \mathrm{m}$ map. Lowest contour is $2 \sigma=8.2 \mathrm{mJy}$, and contours increase in steps of $1 \sigma$

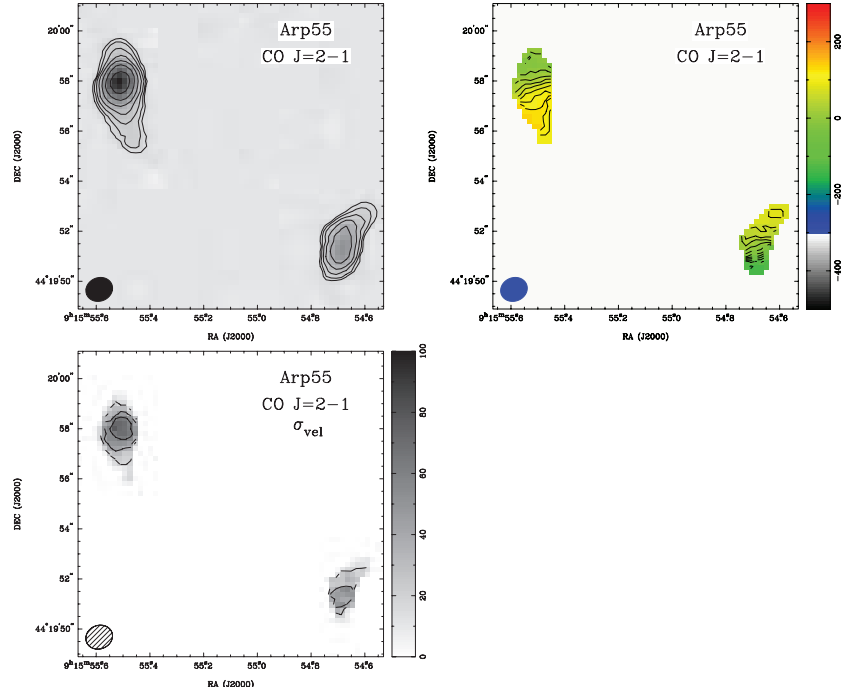

FIG. 12.-Arp $55 \mathrm{CO} J=2-1$ maps. Notation as in Fig. 2. Top left: $\mathrm{CO}$ $J=2-1$ moment 0 map. Lowest contour is $2 \sigma=3.4 \mathrm{Jy} \mathrm{beam}^{-1} \mathrm{~km} \mathrm{~s}^{-1}$, and contours increase by factors of 1.5. Top right: $\mathrm{CO} J=2-1$ moment 1 map. Contours are $20 \mathrm{~km} \mathrm{~s}^{-1} \times(-7,-6,-5,-4,-3,-2,-1,0,1,2,3,4,5,6)$ relative to $c z$. Note that the positive end of the rotation curve in the northeast component peaks at $120 \mathrm{~km} \mathrm{~s}^{-1}$ and then drops to $100 \mathrm{~km} \mathrm{~s}^{-1}$ at the extreme southern end of the emission. Bottom left: $\mathrm{CO} J=2-1$ moment 2 map. Contours are $20 \mathrm{~km} \mathrm{~s}^{-1} \times(1,2,3)$. 

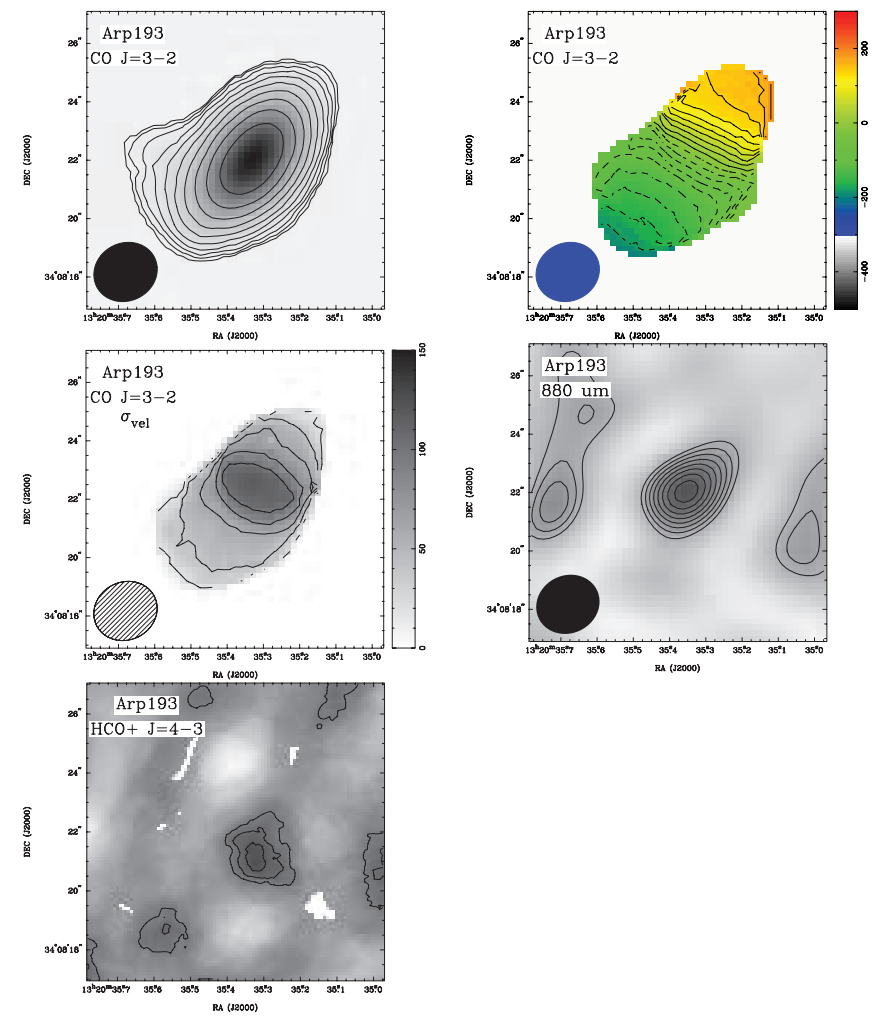

Fig. 13.-Arp $193 \mathrm{CO} J=3-2$ and $880 \mu$ m continuum maps. Notation as in Figs. 2 and 3. Top left: $\mathrm{CO} J=3-2$ moment 0 map. Lowest contour is $2 \sigma=$ $8.5 \mathrm{Jy}$ beam $^{-1} \mathrm{~km} \mathrm{~s}^{-1}$ and contours increase by factors of 1.5. Top right: CO $J=3-2$ moment 1 map. Contours are $20 \mathrm{~km} \mathrm{~s}^{-1} \times(-9,-8,-7,-6,-5,-4$, $-3,-2,-1,0,1,2,3,4,5,6,7,8,9)$ relative to $c z$. Middle left: $\mathrm{CO} J=3-2$ moment 2 map. Contours are $20 \mathrm{~km} \mathrm{~s}^{-1} \times(1,2,3,4,5)$. Middle right: Uncleaned $880 \mu \mathrm{m}$ map. Lowest contour is $2 \sigma=8.0 \mathrm{mJy}$ and contours increase in steps of $1 \sigma$. Sidelobes can be seen at the edges of this figure. Bottom left: Uncleaned $\mathrm{HCO}^{+} J=4-3$ moment 0 map. Lowest contour is $2 \sigma=8.84 \mathrm{Jy}_{\text {beam }}^{-1} \mathrm{~km} \mathrm{~s}^{-1}$ and contours increase by in steps of $1 \sigma$.

(Hinz \& Rieke 2006; Dasyra et al. 2006). In some cases, the lower values found in this paper may be related to the somewhat lower angular resolution of the millimeter-wave $\mathrm{CO}$ observations, as discussed by Hinz \& Rieke (2006). The near-infrared data may also overestimate the velocity dispersion if the $\mathrm{CO}$ bandhead emission is strongly peaked on the nucleus (Hinz \& Rieke 2006). We derive a significantly larger velocity dispersion for IRAS 17208-0014; this may be due to the lower signal-to-noise ratio of the near-infrared spectrum for this galaxy.

\subsubsection{The Relative Strength of the $\mathrm{HCO}^{+} J=4-3$ Emission}

We detect the $\mathrm{HCO}^{+} J=4-3$ line in seven of the 14 galaxies in our sample. Since the weak $\mathrm{HCO}^{+}$emission is extremely compact, we compare the line strength to the peak line strength of the $\mathrm{CO} J=3-2$ line. The $\mathrm{CO} / \mathrm{HCO}^{+}$line ratios are 8 and 16 for Mrk 231 and NGC 2623, respectively, and range from $\leq 7$ to $\leq 32$ for the other five galaxies. Treating all the $\mathrm{HCO}^{+}$lines as detections rather than upper limits, and treating the detections of the eastern and central components of VV114 separately, the average line ratio is 19 with an rms deviation of 9 . However, the true average line ratio for these galaxies is likely somewhat smaller, since many of the $\mathrm{HCO}^{+}$detections are lower limits, because the line emission extends to the edge of the spectral window.

This line ratio is comparable to the average $\mathrm{CO} / \mathrm{HCO}^{+}$line ratio using the $J=1-0$ and $J=3-2$ lines, respectively, determined for six of the galaxies in our sample by Graciá-Carpio et al. (2006), who also find a roughly constant $\mathrm{CO} / \mathrm{HCO}^{+}$line ratio as a function of $L_{\mathrm{FIR}}$. High-resolution $\mathrm{HCO}^{+} J=1-0$ data have been published for four of the galaxies in our sample by Imanishi \& Nakanishi (2006) and Imanishi et al. (2007). Comparing their data with our new measurements yields $\mathrm{HCO}^{+} J=4-3 / J=1-0$ line ratios of about four in both Mrk 231 and Arp 299, while this same line ratio appears to vary dramatically from place to place within $\mathrm{VV} 114$. The $\mathrm{HCN} / \mathrm{HCO}^{+} J=1-0$ line ratio also varies spatially in VV 114 (Imanishi et al. 2007) and in Arp 299 (Imanishi \& Nakanishi 2006), which suggests that there are interesting physical and/or chemical variations in the gas properties in these early-stage mergers. Large variations in the $\operatorname{HCN} J=4-3 / J=$ $1-0$ ratio in a sample of four galaxies are evidence for significant differences in the excitation in the gas phase (Papadopoulos 2007).

The seven galaxies detected in $\mathrm{HCO}^{+}$are the galaxies with strong centrally peaked lines, for which the ratio of the $\mathrm{CO}$ peak flux to the full width at half-maximum of the line is greater than 1 (Table 7 and Figs. 25-29). Thus, it appears that we have detected $\mathrm{HCO}^{+}$in all the galaxies in our sample where we have sufficient sensitivity to detect the peak of the line in a single channel. The fact that the remaining galaxies are not detected may be attributed to insufficient sensitivity; while it is possible that some of these galaxies may be relatively weak in $\mathrm{HCO}^{+}$compared to $\mathrm{CO}$, we have no direct evidence of any such line ratio variations.

\subsubsection{The Relative Strength of the ${ }^{13} \mathrm{CO} J=2-1$ Emission}

Of the nine galaxies observed at $230 \mathrm{GHz}$ with the SMA, we have detected ${ }^{13} \mathrm{CO} J=2-1$ emission in only four galaxies: Arp 299, VV 114, NGC 5331S, and NGC 1614. All of these galaxies except NGC 5331S were also detected in $\mathrm{HCO}^{+}$; the only galaxy that was observed at $230 \mathrm{GHz}$ and detected in $\mathrm{HCO}^{+}$but $n o t$ in ${ }^{13} \mathrm{CO}$ is NGC 2623. This is likely because NGC 2623 is less luminous in CO $J=2-1$ (compare NGC 2623 with NGC 1614 in Table 3). Given the average strength of the ${ }^{13} \mathrm{CO}$ line compared to the $\mathrm{CO}$ line in our four detections, we would not expect to detect any of the remaining galaxies in our sample at the $3 \sigma$ level or better.

\subsection{Published and Archival Single-Dish Data}

In this section, we summarize published single-dish data for the $\mathrm{CO} J=3-2$ line and $880 \mu \mathrm{m}$ continuum from the James Clerk Maxwell Telescope (JCMT). To these published results we add unpublished continuum and spectral-line data taken from the JCMT archive. The sample of single-dish data for the $\mathrm{CO} J=$ $2-1$ line and $1.3 \mathrm{~mm}$ continuum is less complete; the available data will be discussed in a future paper.

\subsubsection{Single-Dish $\mathrm{CO} J=3-2$ Data}

A search of the JCMT archive reveals that many of the galaxies in our sample have been observed in the $\mathrm{CO} J=3-2$ line with the JCMT with a $15^{\prime \prime}$ beam. In addition, we used the JCMT to make small $(7 \times 7)$ Nyquist-sampled maps (program M05AC05) or $2^{\prime}$ Nyquist-sampled maps with the new array receiver HARP-B ( program M07AC11) of many of our galaxies in the $\mathrm{CO} J=3-$ 2 line. Our ultimate goal is to combine these single-dish data with our SMA data to obtain complete maps without any missing spatial frequencies. An example and discussion of this technique as applied to VV 114 is given in Petitpas et al. (G. R. 2008, in preparation). These maps also can be used to estimate the total $\mathrm{CO}$ $J=3-2$ emission from some of our galaxies. By combining our new data with archival data, we can obtain single-dish $\mathrm{CO} J=$ 3-2 spectra for all the galaxies in our sample except NGC 5331.

The quality of the data varies quite significantly from one galaxy to another. For three galaxies (VV 114, NGC 2623, NGC 1614), we have maps covering $35^{\prime \prime}$ on a side that clearly include all the 

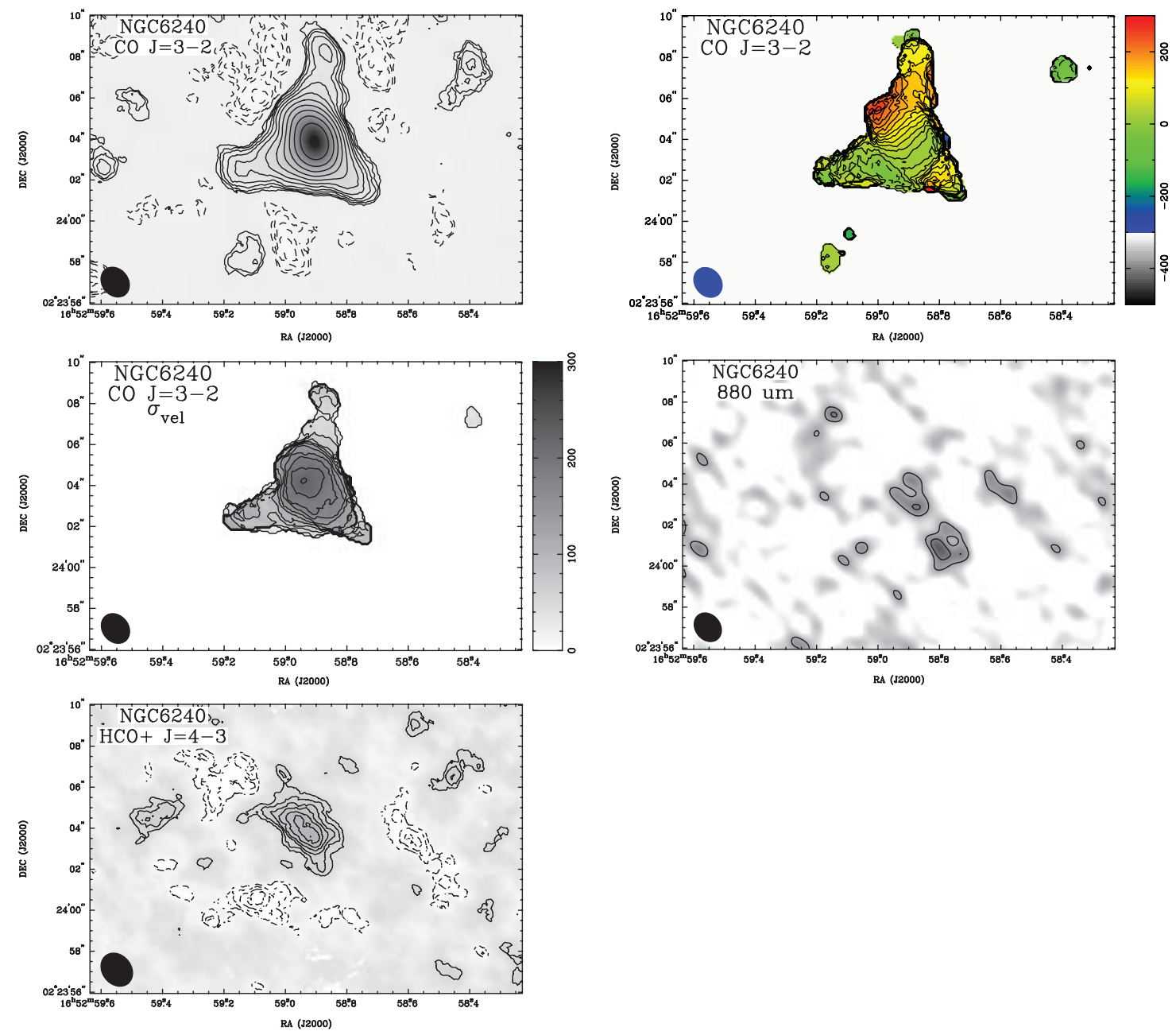

FIG. 14. - NGC $6240 \mathrm{CO} J=3-2$ and $880 \mu$ m continuum maps. Notation as in Figs. 2 and 3. Top left: $\mathrm{CO} J=3-2$ moment 0 map. Lowest contour is $2 \sigma=12.8$ Jy beam ${ }^{-1} \mathrm{~km} \mathrm{~s}^{-1}$, and contours increase by factors of 1.5. Top right: $\operatorname{CO} J=3-2$ moment 1 map. Contours are $20 \mathrm{~km} \mathrm{~s}^{-1} \times(-2,-1,0,1,2,3,4,5,6,7,8,9,10,11,12,13)$ relative to $c z$. Middle left: $\mathrm{CO} J=3-2$ moment 2 map. Contours are $20 \mathrm{~km} \mathrm{~s}^{-1} \times(1,2,3,4,5,6,7,8,9,10,11)$. Middle right: Uncleaned $880 \mu \mathrm{m}$ map. Lowest contour is $2 \sigma=17 \mathrm{mJy}$, and contours increase in steps of $1 \sigma$. Bottom left: Uncleaned $\mathrm{HCO}^{+} J=4-3$ moment 0 map. Lowest contour is $\pm 2 \sigma=10.22 \mathrm{Jy} \mathrm{beam}^{-1} \mathrm{~km} \mathrm{~s}^{-1}$, and contours increase in steps of $1 \sigma$.

emission from the galaxy. For Arp 299 and NGC 5258, we have maps covering $2^{\prime}$ on a side but with bad baselines in some of the outer pixels; in addition, these data needed to be scaled up by a factor of 1.25 to make observations of spectral-line standards consistent with previous results. We also adopt a higher calibration uncertainty (30\%) for these data sets. Mrk 273, Mrk 231, IRAS 10565+2448, UGC 5101, IRAS 17208-0014, NGC 6240, and Arp 193 were all observed in a single coordinated program in 1999 (program M99AN19, PI: Papadopoulos) with many observations of calibrators to check for calibration consistency. Arp 55 has only a single spectrum centered roughly on the southwestern source that is visible in our interferometric maps (Fig. 11). This flux measurement must be considered a lower limit since this pointing would detect very little emission from the stronger northern source. For NGC 5257, there is a single spectrum published in Yao et al. (2003). We reduced these data again ( program M01 AC03, PI: Seaquist), including the calibration data, and there appear to be some problems with the calibration during these observations. The main beam efficiency is $20 \%$ lower than the standard value, and the observations of the spectral line standards are also lower by about a similar amount. Although we have corrected for these effects in calculating the CO flux, the calibration must be considered more uncertain for this galaxy.
In converting the single-dish fluxes from their intrinsic units of kelvins $\left(T_{A}^{*}\right) \mathrm{km} \mathrm{s}^{-1}$ to Jy $\mathrm{km} \mathrm{s}^{-1}$, we assume an aperture efficiency of 0.5 , which gives a scaling factor of $31.3 \mathrm{Jy} \mathrm{K}^{-1}$. All of the galaxies in our sample that have not been mapped are sufficiently compact that we assume a point-source distribution in converting from kelvins to janskys. The exception is Arp 55, for which we assume the emission fills the main beam of the telescope and thus divide by the main beam efficiency $\eta_{\mathrm{MB}}=0.63$ before multiplying by $31.3 \mathrm{Jy} \mathrm{K}^{-1}$. For the four galaxies with small maps, each spectrum was converted to Jy km s${ }^{-1}$ using the point-source scaling factor, and then the spectra were summed and normalized by the number of pixels per $15^{\prime \prime}$ beam in the map to obtain the final integrated flux. The resulting single-dish CO $J=3-2$ fluxes are given in Table 4 .

\subsubsection{Single-Dish $880 \mu \mathrm{m}$ Data}

Published 850 or $800 \mu \mathrm{m}$ fluxes from the JCMT are available for all the galaxies in our sample except NGC 5331 and Arp 299. Data presented by Rigopoulou et al. (1996) for Mrk 231, Mrk 273, and UGC 5101 were obtained with the single-pixel bolometer UKT14 at $800 \mu \mathrm{m}$, while data from Klaas et al. (2001) for IRAS 17208-0014 and NGC 6240 and Dunne et al. (2000) for the remaining galaxies were obtained with the bolometer array SCUBA 


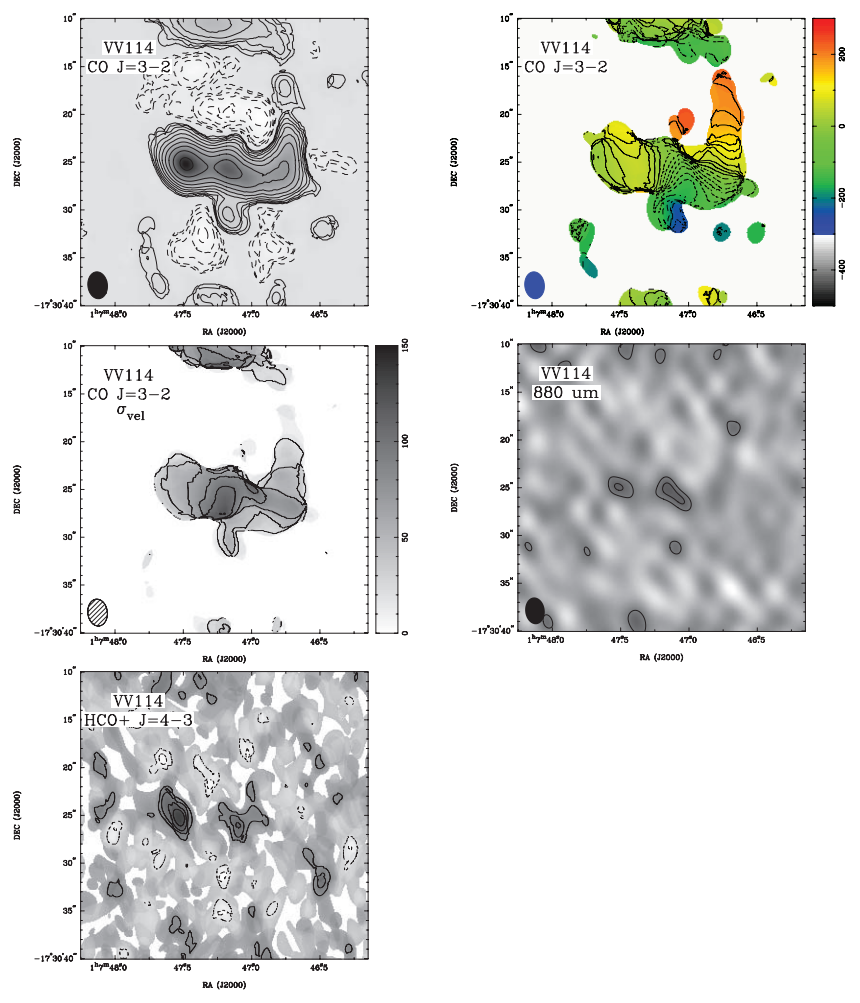

FIG. 15.- VV $114 \mathrm{CO} J=3-2$ and $880 \mu \mathrm{m}$ continuum maps. Notation as in Figs. 2 and 3. Top left: $\mathrm{CO} J=3-2$ moment 0 map. Lowest contour is $\pm 2 \sigma=$ $7.0 \mathrm{Jy} \mathrm{beam}^{-1} \mathrm{~km} \mathrm{~s}^{-1}$, and contours increase by factors of 1.5 . The regions of positive emission at the extreme northern and southern edges of the image are artifacts. Top right: $\mathrm{CO} J=3-2$ moment 1 map. Contours are $20 \mathrm{~km} \mathrm{~s}^{-1} \times(-12$, $-11,-10, \ldots,-2,-1,0,1,2, \ldots, 9,10,11)$ relative to $c z$. Middle left: $\mathrm{CO}$ $J=3-2$ moment 2 map. Contours are $20 \mathrm{~km} \mathrm{~s}^{-1} \times(1,2,3,4,5)$. Middle right: Uncleaned $880 \mu \mathrm{m}$ map. Lowest contour is $2 \sigma=7.8 \mathrm{mJy}$, and contours increase in steps of $1 \sigma$. Bottom left: $\mathrm{HCO}^{+} J=4-3$ moment 0 map. Lowest contour is $\pm 2 \sigma=3.68 \mathrm{Jy} \mathrm{beam}^{-1} \mathrm{~km} \mathrm{~s}^{-1}$, and contours increase in steps of $1 \sigma$. The eastern component contains $60 \%$ of the flux given in Table 3 .

at $850 \mu \mathrm{m}$. In addition, SCUBA data for VV 114, NGC 5331, and Arp 299 are available in the JCMT archive. These data were processed into images using an automatic pipeline (Di Francesco et al. 2008) and the fluxes were measured by us from those images.

We then rescaled the fluxes by dividing by a factor of $1.13 \pm$ $0.02(850 \mu \mathrm{m})$ or $1.40 \pm 0.06(800 \mu \mathrm{m})$ to obtain an estimate of the continuum flux at $880 \mu \mathrm{m}$. These scaling factors assume a dust emissivity $\beta=1.5 \pm 0.5$. The resulting single-dish $880 \mu \mathrm{m}$ continuum fluxes are given in Table 5. The published uncertainties vary somewhat in their meaning from author to author. Klaas et al. (2001) simply adopt an uncertainty of 30\%. The errors given by Rigopoulou et al. (1996) and for VV 114, NGC 5331, and Arp 299 are purely statistical measurement errors with no calibration uncertainty added in. The errors given by Dunne et al. (2000) include a calibration uncertainty of $10 \%(15 \%$ for NGC 5257 and NGC 5258). In comparing the single-dish and SMA fluxes, we have adopted a $20 \%$ calibration uncertainty for each telescope and adjusted the published uncertainties for the singledish data accordingly.

\subsection{Comparison of Interferometric and Single-Dish Fluxes}

A comparison of the $\mathrm{CO} J=3-2$ fluxes recovered by the SMA with the fluxes seen by the JCMT shows that the interferometric data miss a significant fraction of the $\mathrm{CO} J=3-2$ emission for eight of the galaxies in our sample. In these galaxies, we are typically missing $50 \%$ of the total single-dish flux. However, only
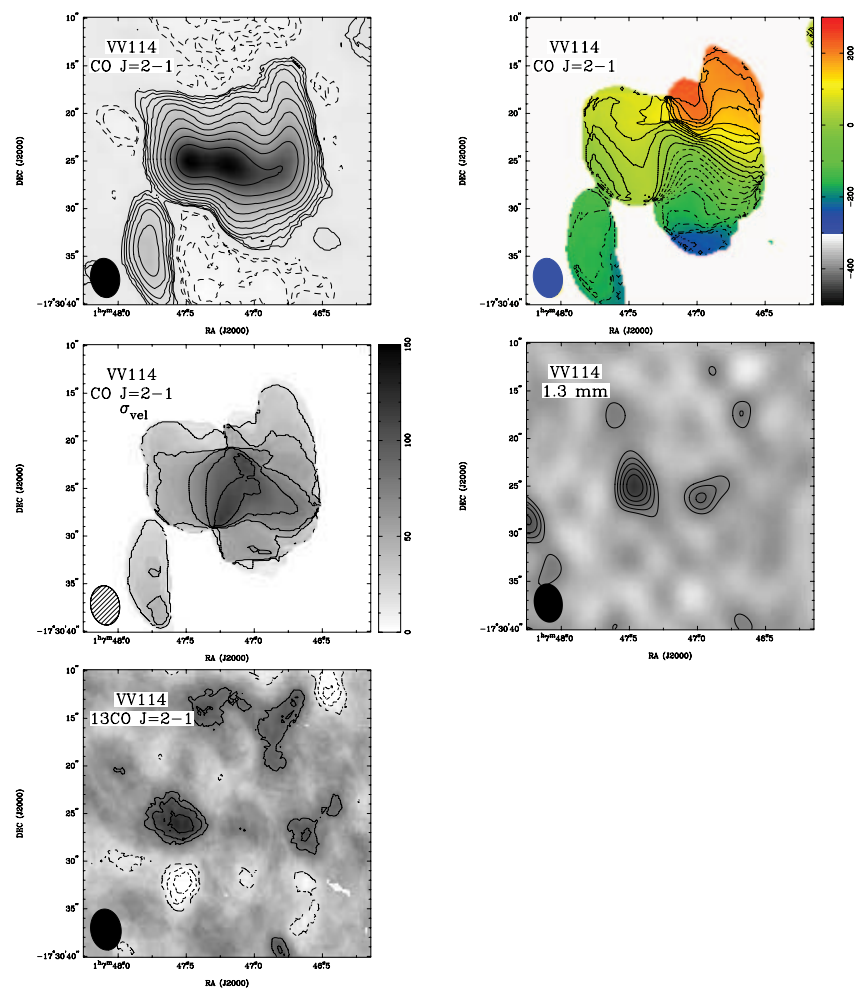

FIG. 16.-VV $114 \mathrm{CO} J=2-1$ and $1.3 \mathrm{~mm}$ continuum maps. Notation as in Fig. 2 and 10. Top left: CO $J=2-1$ moment 0 map. Lowest contour is $\pm 2 \sigma=$ $3.5 \mathrm{Jy} \mathrm{beam}^{-1} \mathrm{~km} \mathrm{~s}^{-1}$, and contours increase by factors of 1.5. (This image also has strong artifacts similar to those seen in Fig. 15, but they are outside the field of view shown here.) Top right: CO $J=2-1$ moment 1 map. Contours are $20 \mathrm{~km} \mathrm{~s}^{-1} \times$ $(-12,-11,-10, \ldots,-2,-1,0,1,2, \ldots, 9,10,11)$ relative to $c z$. Middle left: $\mathrm{CO} J=2-1$ moment 2 map. Contours are $20 \mathrm{~km} \mathrm{~s}^{-1} \times(1,2,3,4,5)$. Middle right: Uncleaned $1.3 \mathrm{~mm}$ map. Lowest contour is $2 \sigma=2.6 \mathrm{mJy}$, and contours increase in steps of $1 \sigma$. Bottom left: ${ }^{13} \mathrm{CO} J=2-1$ moment 0 map. Lowest contour is $\pm 2 \sigma=3.2 \mathrm{Jy} \mathrm{beam}^{-1} \mathrm{~km} \mathrm{~s}^{-1}$ and contours increase in steps of $1 \sigma$.

for three galaxies in our sample, Arp 299, VV 114, and NGC 1614 , does this missing flux manifest itself as significant negative bowls in the moment maps (Figs. 9, 15, and 23). These are the three galaxies with the largest absolute amount of missing flux, and so it makes sense that they are the ones in which the missing flux leaves a detectable negative signal in the maps.

We can also calculate the percentage of continuum flux missed by the interferometric observations by comparing the continuum flux detected with the SMA to the single-dish continuum flux detected with the JCMT. In doing this calculation, we also correct for the contribution of the CO $J=3-2$ line within the SCUBA filter using the formula given by Seaquist et al. (2004). The results given in Table 5 show that, while the interferometric observations detect all of the single-dish continuum flux in four galaxies (and possibly in a fifth, NGC 5258, if the off-nuclear continuum source is not time variable), for the remaining nine galaxies typically $50 \%-80 \%$ of the continuum flux is missed by the interferometer.

This result is somewhat surprising, as the few sources studied previously at millimeter wavelengths at high resolution show quite compact continuum emission. For example, Sakamoto et al. (1999) detect all the $1.3 \mathrm{~mm}$ continuum emission in Arp 220 and find it is contained in two compact components associated with the merging nuclei. For Mrk 231, Downes \& Solomon (1998) also detect all of the $1.3 \mathrm{~mm}$ continuum emission seen in single-dish data (Carico et al. 1992), although in this case the emission is thought to be primarily nonthermal emission from the central AGN, with thermal emission from dust contributing only $20 \%$ of 

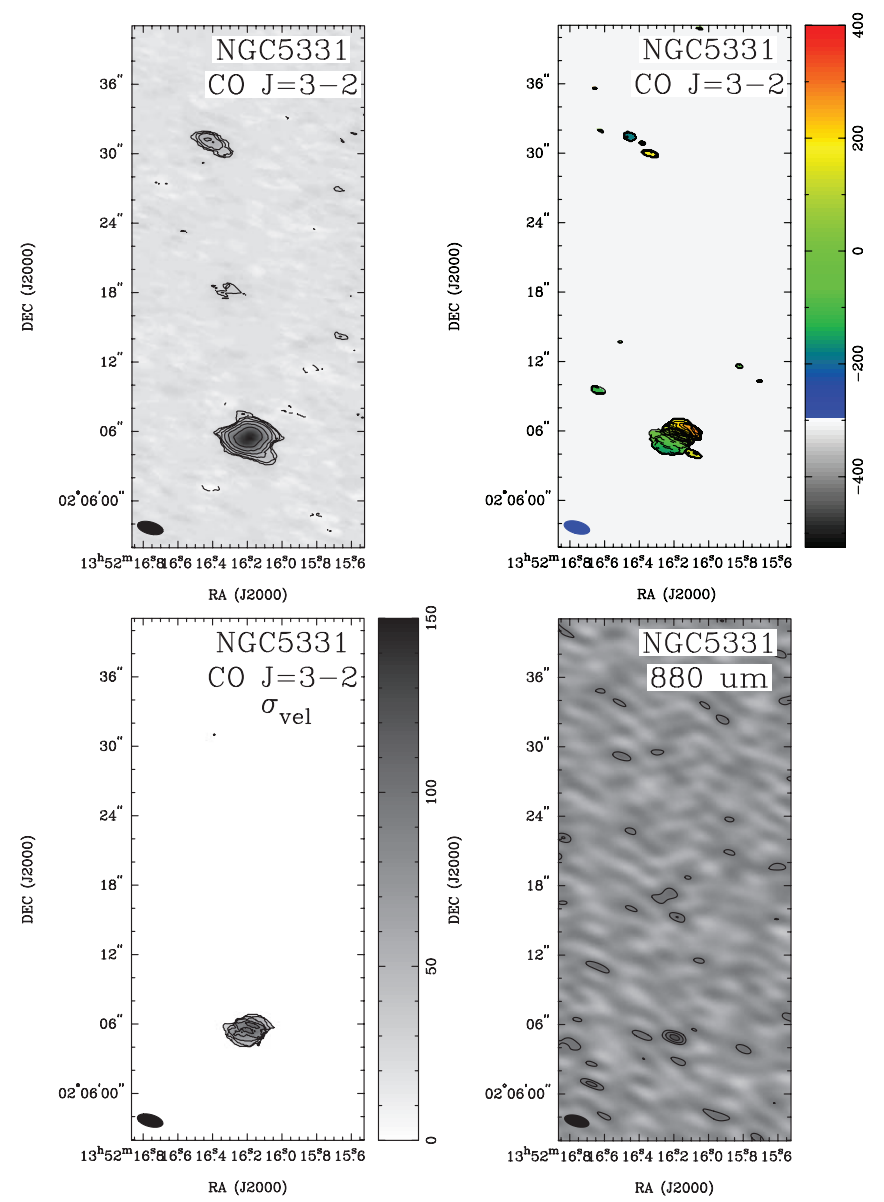

FIG. 17.-NGC $5331 \mathrm{CO} J=3-2$ and $880 \mu \mathrm{m}$ continuum maps. Notation as in Fig. 2. Top left: CO $J=3-2$ moment 0 map. Lowest contour is $\pm 2 \sigma=$ $21.6 \mathrm{Jy}^{\text {beam }}{ }^{-1} \mathrm{~km} \mathrm{~s}^{-1}$, and contours increase by factors of 1.5. Top right: $\mathrm{CO}$ $J=3-2$ moment 1 map. Contours are $20 \mathrm{~km} \mathrm{~s}^{-1} \times(-8,-7,-6,-5, \ldots,-2$, $-1,0,1,2, \ldots, 14,15,16)$ relative to $c z$. Bottom left: $\mathrm{CO} J=3-2$ moment 2 map. Contours are $20 \mathrm{~km} \mathrm{~s}^{-1} \times(1,2,3,4,5)$. Bottom right: Uncleaned $880 \mu \mathrm{m}$ map. Lowest contour is $2 \sigma=12 \mathrm{mJy}$, and contours increase in steps of $1 \sigma$.

the total flux. Mrk 273 and Arp 193 have also been detected at $1.3 \mathrm{~mm}$ by Downes \& Solomon (1998) but there are no single-dish data for these galaxies that can be used to determine the total flux.

The large amount of missing continuum flux in many of our sample galaxies suggests that a significant fraction of the $880 \mu \mathrm{m}$ emission occurs on moderately large spatial scales even in systems with very luminous compact cores. (The $15^{\prime \prime}$ beam of the JCMT subtends 3-14 kpc at the distances of the galaxies in our sample.) A plot of the percentage of flux missing at $880 \mu \mathrm{m}$ versus the percentage missing in the CO $J=3-2$ line (not shown) shows reasonable agreement for all galaxies, although on average there is a slightly higher percentage of the total flux missing in the continuum images. The good agreement between the percentage of missing flux seen in the $\mathrm{CO} J=3-2$ line and the $880 \mu \mathrm{m}$ continuum suggests that the missing continuum flux comes from dust emission associated with molecular gas in the more extended disks of the galaxies. Such gas could be associated with a disk-wide starburst (as opposed to a nuclear starburst) or more quiescent star formation as is seen in less luminous spiral galaxies. The difference between our results and previous results for Arp 220 and Mrk 231 is likely due to the fading contribution from dust emission at $1.3 \mathrm{~mm}$ combined with the importance of nonthermal and possibly free-free emission in these two galaxies.

\section{THE GAS-TO-DUST MASS RATIO IN THE NUCLEAR REGIONS}

We can use our new CO $J=3-2$ and $880 \mu$ m data to estimate the gas and dust masses, and in particular the gas-to-dust mass ratio, in the central regions of these LIRGs. The gas-to-dust ratio is interesting because it allows us to probe the physical properties of the interstellar medium on kiloparsec scales in regions of galaxies with intense heating from starburst activity and (perhaps) vigorous accretion. Alternatively, it can be used to provide an independent check of the gas masses determined from the $\mathrm{CO}$ emission lines. The gas mass in the central region is a critical quantity for understanding the evolution of these galaxies, as it determines the fuel for the current activity, be it a central starburst or accretion onto an active nucleus.

\subsection{Gas and Dust Mass Calculations}

We have calculated the dust mass from the $880 \mu \mathrm{m}$ flux assuming a dust emissivity at $880 \mu \mathrm{m}, \kappa=0.9 \mathrm{~cm}^{2} \mathrm{~g}^{-1}$, appropriate for molecular cloud envelopes (Henning et al. 1995; Johnstone et al. 2000). With this assumption, the dust mass is given by

$$
M_{\text {dust }}=74,220 S_{880} D_{L}^{2}\left(\exp \left(17 / T_{D}\right)-1\right) / \kappa\left(M_{\odot}\right),
$$

where $S_{880}$ is the $880 \mu \mathrm{m}$ flux in janskys and $D_{L}$ is the luminosity distance in megaparsecs. We calculated the dust temperature, $T_{D}$, from optically thin modified blackbody fits to published global photometric data between 60 and $800 \mu \mathrm{m}$, following the procedure of Klaas et al. (2001).

Our derived temperatures for Mrk 231, Mrk 273, UGC 5101, and NGC 6240 are between 5 and $10 \mathrm{~K}$ warmer than the temperature of the cold component derived by Armus et al. (2007). However, our temperatures are generally in good agreement (except for Mrk 231, for which we are $8 \mathrm{~K}$ colder) with the values derived by Yang \& Phillips (2007) in fits including new data at $350 \mu \mathrm{m}$.

To calculate the molecular hydrogen gas mass from the $\mathrm{CO} J=$ 3-2 emission line, we need to know the CO-to- $\mathrm{H}_{2}$ conversion factor, $X_{\mathrm{CO}}$. While this conversion factor is now reasonably well determined for normal galaxies (Strong et al. 1988; Wilson \& Scoville 1990; Strong \& Mattox 1996; Dame et al. 2001; Rosolowsky et al. 2003), it has been clear for many years that the standard conversion factor does not apply to starbursting systems like the galaxies studied here (Bryant \& Scoville 1996; Downes \& Solomon 1998). In addition, the conversion factor has been determined for the CO $J=1-0$ line only. To apply the appropriate conversion factor to our CO $J=3-2$ data also requires us to know or assume an appropriate $\mathrm{CO} J=3-2 / J=1-0$ line ratio.

To calculate the $\mathrm{H}_{2}$ gas mass, we adopt the revised conversion factor advocated by Downes \& Solomon (1998) $M_{\mathrm{H}_{2}}=0.8 L_{\mathrm{CO}}^{\prime}(1-$ 0 ), where $M_{\mathrm{H}_{2}}$ is the $\mathrm{H}_{2}$ gas mass in $M_{\odot}$ and $L_{\mathrm{CO}}^{\prime}(1-0)$ is the luminosity of the $\mathrm{CO} J=1-0$ line in $\mathrm{K} \mathrm{km} \mathrm{s}^{-1} \mathrm{pc}^{2}$. [This equation corresponds to a CO-to- $\mathrm{H}_{2}$ conversion factor of $0.5 \times$ $10^{20} \mathrm{H}_{2} \mathrm{~cm}^{-2}\left(\mathrm{~K} \mathrm{~km} \mathrm{~s}^{-1}\right)^{-1}$.] To convert our measurements in Jy $\mathrm{km} \mathrm{s}^{-1}$ to units of $\mathrm{K} \mathrm{km} \mathrm{s}^{-1} \mathrm{pc}^{2}$, we use the equation

$$
\begin{aligned}
\frac{L^{\prime}}{\mathrm{K} \mathrm{km} \mathrm{s}^{-1} \mathrm{pc}^{2}}= & 3.2546 \times 10^{7}\left(\frac{S_{\mathrm{CO}}}{\mathrm{Jy} \mathrm{km} \mathrm{s}^{-1}}\right) \\
& \times\left(\frac{D_{L}}{\mathrm{Mpc}}\right)^{2}\left(\frac{\nu_{0}}{\mathrm{GHz}}\right)^{-2}(1+z)^{-1},
\end{aligned}
$$

where $S_{\mathrm{CO}}$ is the CO integrated intensity, $\nu_{0}$ is the rest frequency of the $\mathrm{CO}$ transition, and $z$ is the redshift. To determine the $\mathrm{CO}$ $J=3-2 / J=1-0$ line ratio, we compared the interferometric and single-dish CO $J=3-2$ data discussed here with published 


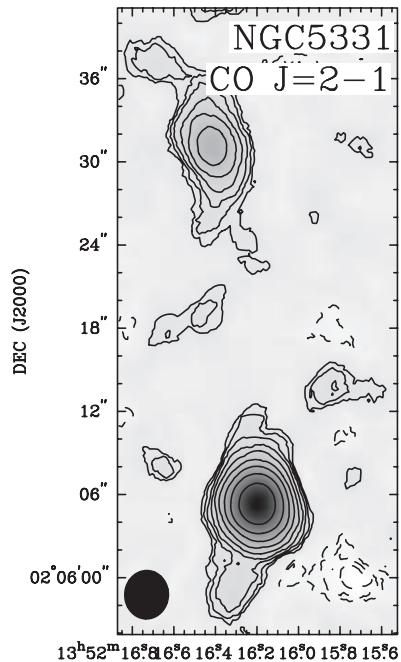

RA (J2000)

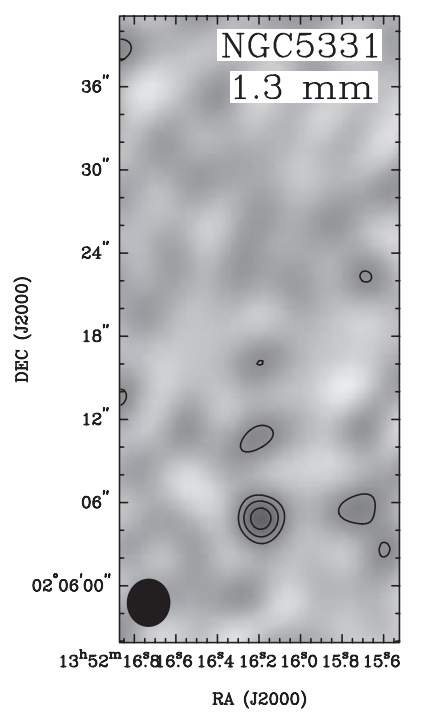

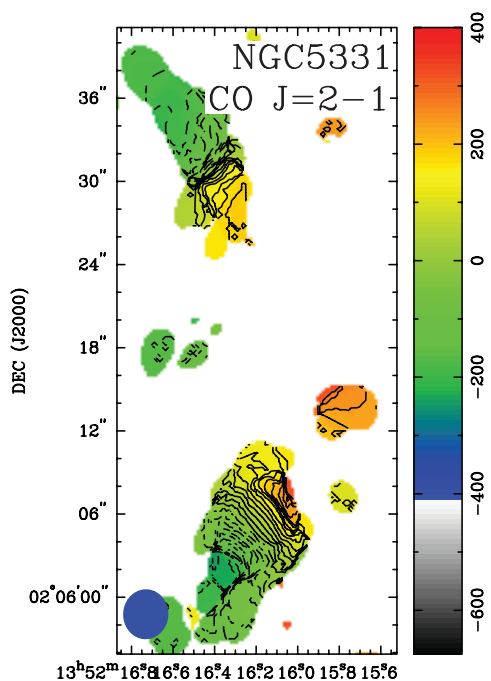

RA (J2000)

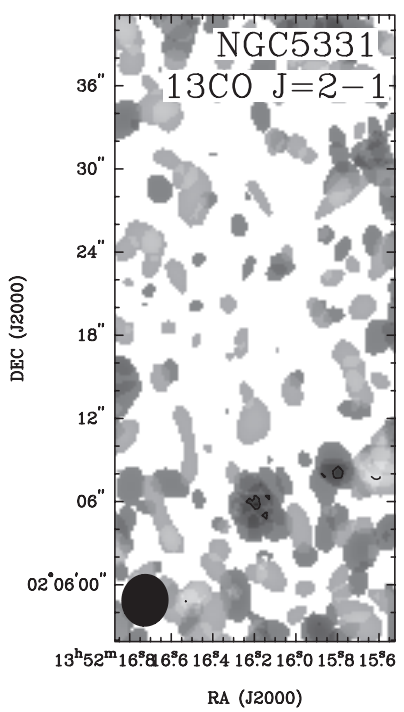

FIG. 18. - NGC $5331 \mathrm{CO} J=2-1$ and $1.3 \mathrm{~mm}$ continuum maps. Notation as in Fig. 2 and 10. Top left: CO $J=2-1 \mathrm{moment} 0 \mathrm{map}$. Lowest contour is $\pm 2 \sigma=5.1 \mathrm{Jy}$

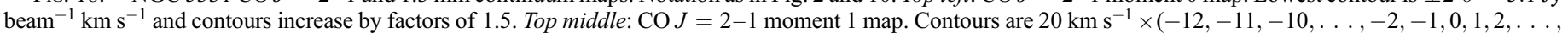

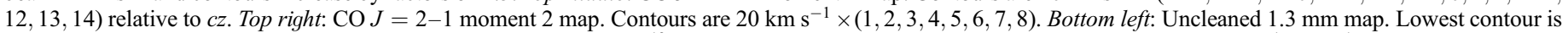
$2 \sigma=2.6 \mathrm{mJy}$, and contours increase in steps of $1 \sigma$. Bottom middle: ${ }^{13} \mathrm{CO} \mathrm{J}=2-1$ moment 0 map. The contour is $2 \sigma=4.6 \mathrm{Jy}$ beam ${ }^{-1} \mathrm{~km} \mathrm{~s}^{-1}$.

interferometric $\mathrm{CO} J=1-0$ data of similar angular resolution (Downes \& Solomon 1998), and single-dish data for Mrk 231 (Solomon et al. 1997) and Mrk 273 (Gao \& Solomon 2004). The average single-dish line ratio for the latter two galaxies is 0.48 , while the average interferometric line ratio is 0.56 , where both values are calculated on the temperature scale. Assuming absolute calibration uncertainties of $20 \%$ for both data sets, these two values agree well, and so we adopt an average CO $J=3-2$ / $J=1-0$ line ratio of 0.5 in calculating the $\mathrm{H}_{2}$ gas mass. Thus, the $\mathrm{H}_{2}$ gas mass is calculated via

$$
M_{\mathrm{H}_{2}}=1.6 L_{\mathrm{CO}}^{\prime}(3-2)
$$

where $L_{\mathrm{CO}}^{\prime}(3-2)$ is the luminosity of the $\mathrm{CO} J=3-2$ line in $\mathrm{K} \mathrm{km} \mathrm{s}^{-1} \mathrm{pc}^{2}$. (Note that to obtain the total mass in molecular gas, $M_{\mathrm{mol}}, M_{\mathrm{H}_{2}}$ would need to be multiplied by a factor of 1.36 to account for helium.) Gas and dust masses and the gas-to-dust mass ratio calculated from the total detected $\mathrm{CO} J=3-2$ and $880 \mu \mathrm{m}$ luminosity of each galaxy or galaxy component are given in Table 6, while similar quantities calculated from the peak $\mathrm{CO}$
$J=3-2$ emission and $880 \mu \mathrm{m}$ flux in a single beam are given in Table 7 .

\subsection{Uncertainties in Gas and Dust Masses}

The primary source of uncertainty in the $\mathrm{H}_{2}$ masses we have calculated is the CO-to- $\mathrm{H}_{2}$ conversion factor. We have adopted here the value advocated by Downes \& Solomon (1998) as being the most appropriate for ULIRGs. The Milky Way value for this conversion factor is about 5 times larger (Solomon et al. 1997). Thus, if some of the galaxies in our sample, particularly the less luminous ones, have an interstellar medium more similar to that of the Milky Way than that of ULIRGs, the $\mathrm{H}_{2}$ masses in Tables 6 and 7 could be underestimated by up to a factor of 5. Detailed radiative transfer and dynamical modeling can reduce the uncertainty in this factor by probing the physical conditions directly (Iono et al. 2007) and may provide an independent determination of the CO-to- $\mathrm{H}_{2}$ conversion factor, and will be presented in a future paper.

A second source of uncertainty comes in our adopted value of the $\operatorname{CO} J=3-2 / J=1-0$ line ratio. This uncertainty is probably 

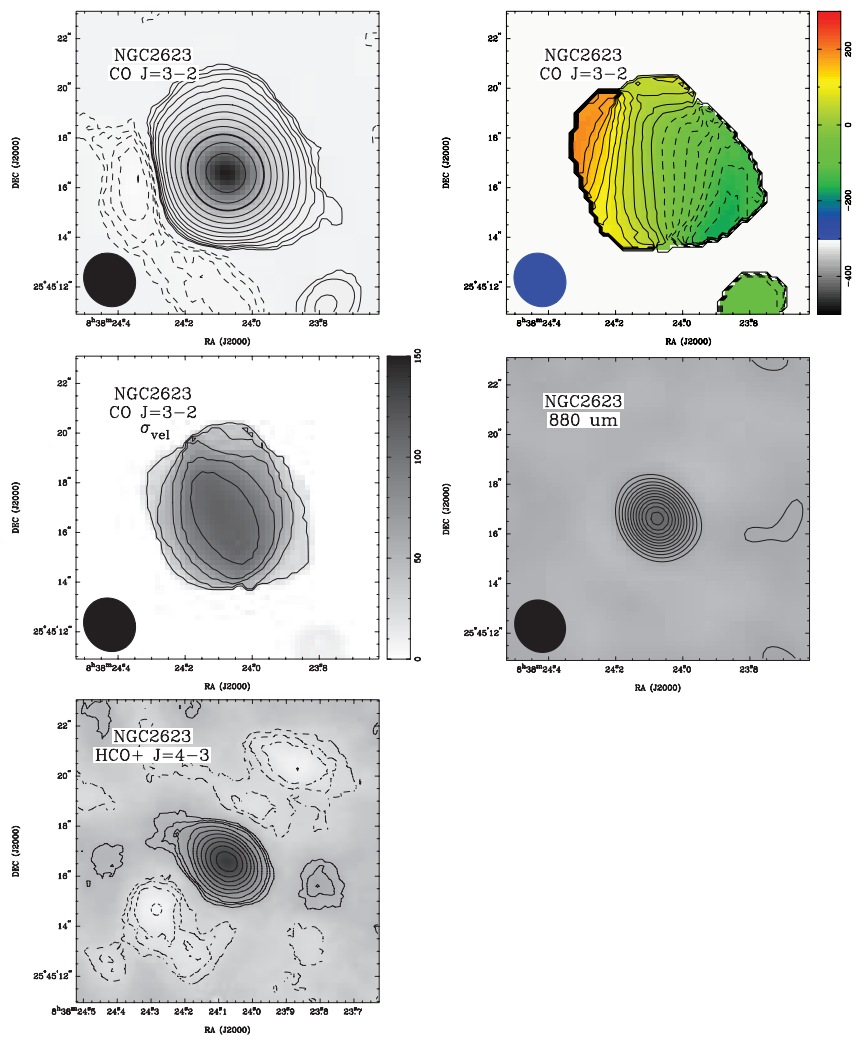

FIG. 19.-NGC $2623 \mathrm{CO} J=3-2$ and $880 \mu$ m continuum maps. Notation as in Figs. 2 and 3. Top left: $\mathrm{CO} J=3-2$ moment 0 map. Lowest contour is $\pm 2 \sigma=$ $2.7 \mathrm{Jy}_{\text {beam }}^{-1} \mathrm{~km} \mathrm{~s}^{-1}$ and contours increase by factors of 1.5. Top right: CO $J=3-2$ moment 1 map. Contours are $20 \mathrm{~km} \mathrm{~s}^{-1} \times(-8,-7,-6,-5,-4,-3$, $-2,-1,0,1,2,3,4,5,6,7,8,9)$ relative to $c z$. Middle left: $\mathrm{CO} J=3-2$ moment 2 map. Contours are $20 \mathrm{~km} \mathrm{~s}^{-1} \times(1,2,3,4,5)$. Middle right: Uncleaned $880 \mu \mathrm{m}$ map. Lowest contour is $2 \sigma=4 \mathrm{mJy}$, and contours increase in steps of $2 \sigma$. Bottom left: Uncleaned $\mathrm{HCO}^{+} J=4-3$ moment 0 map. Lowest contour is $\pm 2 \sigma=$ $2.60 \mathrm{Jy}$ beam ${ }^{-1} \mathrm{~km} \mathrm{~s}^{-1}$, and contours increase in steps of $1 \sigma$ to $4 \sigma$ and then by steps of $2 \sigma$.
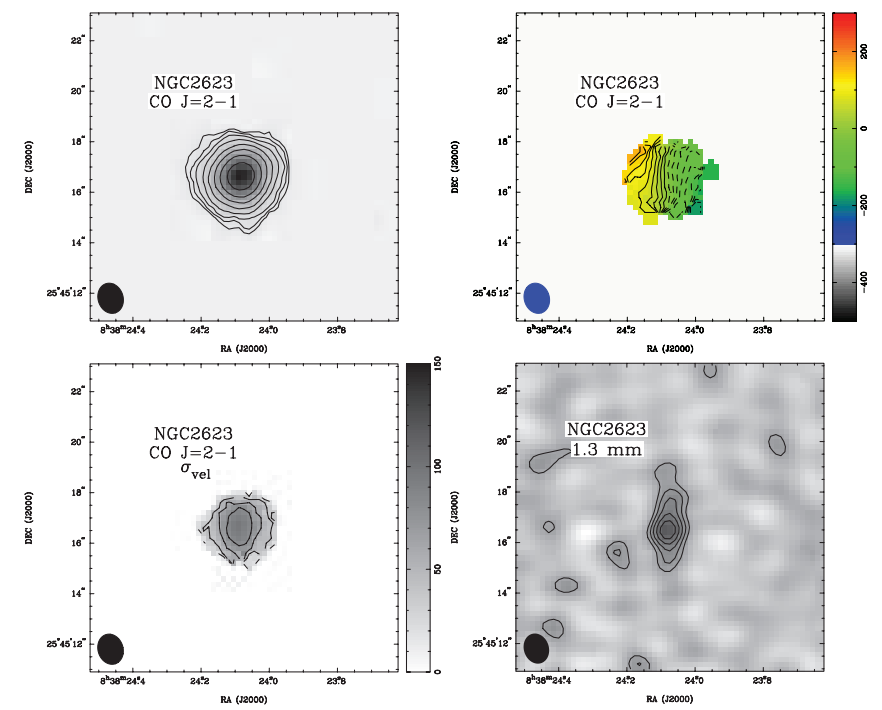

Fig. 20.-NGC $2623 \mathrm{CO} J=2-1$ and $1.3 \mathrm{~mm}$ continuum maps. Notation as in Fig. 2. Top left: $\mathrm{CO} J=2-1$ moment 0 map. Lowest contour is $2 \sigma=$ $4.0 \mathrm{Jy}$ beam $^{-1} \mathrm{~km} \mathrm{~s}^{-1}$, and contours increase by factors of 1.5 . Top right: CO $J=2-1$ moment 1 map. Contours are $20 \mathrm{~km} \mathrm{~s}^{-1} \times(-9,-8,-7,-6,-5,-4$, $-3,-2,-1,0,1,2,3,4,5,6,7,8)$ relative to $c z$. Middle left: $\mathrm{CO} J=2-1 \mathrm{mo-}$ ment 2 map. Contours are $20 \mathrm{~km} \mathrm{~s}^{-1} \times(1,2,3,4)$. Bottom right: Uncleaned $1.3 \mathrm{~mm}$ map. Lowest contour is $2 \sigma=2.6 \mathrm{mJy}$ and contours increase in steps of $1 \sigma$.
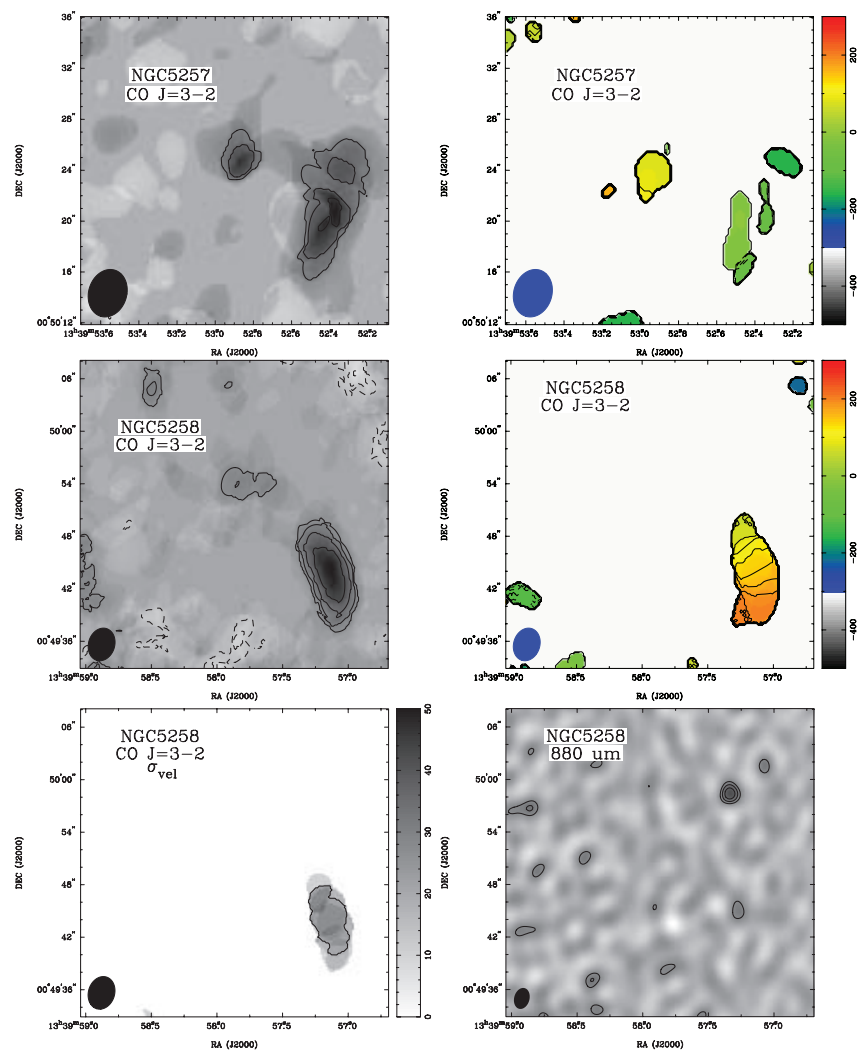

FIG. 21.-NGC 5257/8 CO $J=3-2$ and $880 \mu \mathrm{m}$ continuum maps. Notation as in Fig. 2. Top left: CO $J=3-2$ moment 0 map for NGC 5257. Lowest contour is $2 \sigma=22.1 \mathrm{Jy}_{\text {beam }}^{-1} \mathrm{~km} \mathrm{~s}^{-1}$, and contours increase by factors of 1.5. Top right: CO $J=3-2$ moment 1 map for NGC 5257. Contours are $20 \mathrm{~km} \mathrm{~s}^{-1} \times$ $(0,1,2,34,5)$ relative to $c z$. Middle left: $\mathrm{CO} J=3-2$ moment 0 map for NGC 5258. Lowest contour is $\pm 2 \sigma=33.7 \mathrm{Jy}_{\text {beam }}^{-1} \mathrm{~km} \mathrm{~s}^{-1}$, and contours increase by factors of 1.5. Middle right: $\mathrm{CO} J=3-2$ moment 1 map for NGC 5258. Contours are $20 \mathrm{~km} \mathrm{~s}^{-1} \times(3,4,5,6,7,8,9,10)$ relative to $c z$. Bottom left: $\mathrm{CO} J=3-2$ moment 2 map for NGC 5258 . Contour is $20 \mathrm{~km} \mathrm{~s}^{-1}$. Bottom right: Uncleaned $880 \mu \mathrm{m}$ map. Lowest contour is $2 \sigma=30 \mathrm{mJy}$ and contours increase in steps of $1 \sigma$.

not as large as the uncertainty caused by the CO-to- $\mathrm{H}_{2}$ conversion factor, as the line ratio we have adopted as being appropriate for Mrk 231 and Mrk 273 agrees very well with the line ratio seen in star-forming regions in more normal spiral galaxies such as M33 (Wilson et al. 1997) and in the more highly inclined galaxies from the sample of Dumke et al. (2001). However, many of the galaxies in the Dumke et al. (2001) sample have line ratios of $1-1.4$, so if it introduces any bias, our adopted line ratio would overestimate the $\mathrm{H}_{2}$ mass by a factor of at most $2-3$. In addition, any systematic changes in the line ratio with luminosity or evolutionary phase would introduce similar systematic effects into the gas-to-dust mass ratio. A spatially resolved comparison with highresolution $\mathrm{CO} J=1-0$ images of these galaxies, which would reduce the uncertainty due to the line ratio, will be presented in a future paper (see also Iono et al. 2007).

One major source of uncertainty in the dust masses we have calculated is the dust temperature. An alternative to assuming optically thin dust with emissivity varying as $\nu^{\beta}$ is to assume that the 60 and $100 \mu \mathrm{m}$ fluxes can be fit with an optically thick blackbody as in Solomon et al. (1997). This method gives, on average, dust temperatures that are 1.5 times larger and dust masses that are 0.55 times smaller than the values given here. The net result of assuming optically thick emission in the far-infrared would be to increase the gas-to-dust mass ratio by about a factor of 2 . Conversely, if we have overestimated the dust temperature by using 

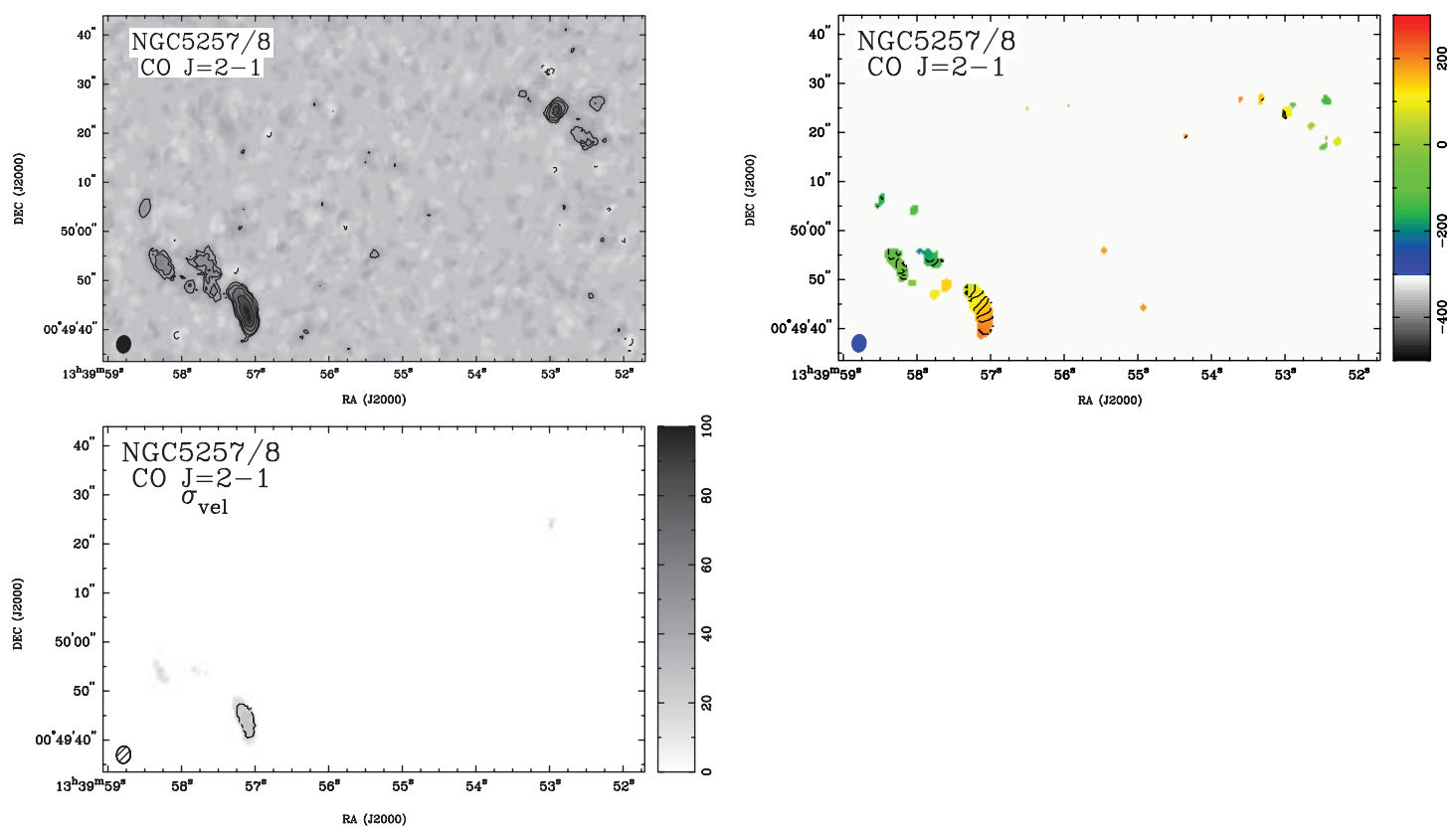

Fig. 22.-NGC 5257/8 CO $J=2-1$ maps. Notation as in Fig. 2. Top left: $\mathrm{CO} J=2-1$ moment 0 map. Lowest contour is $2 \sigma=10.8 \mathrm{Jy} \mathrm{beam}^{-1} \mathrm{~km} \mathrm{~s}^{-1}$, and contours increase by factors of 1.5. Top right: $\mathrm{CO} J=2-1$ moment 1 map. Contours are $20 \mathrm{~km} \mathrm{~s}^{-1} \times(-8,-7,-6, \ldots,-2,-1,0,1,2, \ldots, 8,9,10)$ relative to cz. Middle left: $\mathrm{CO}$ $J=2-1$ moment 2 map. Contour is $20 \mathrm{~km} \mathrm{~s}^{-1}$.
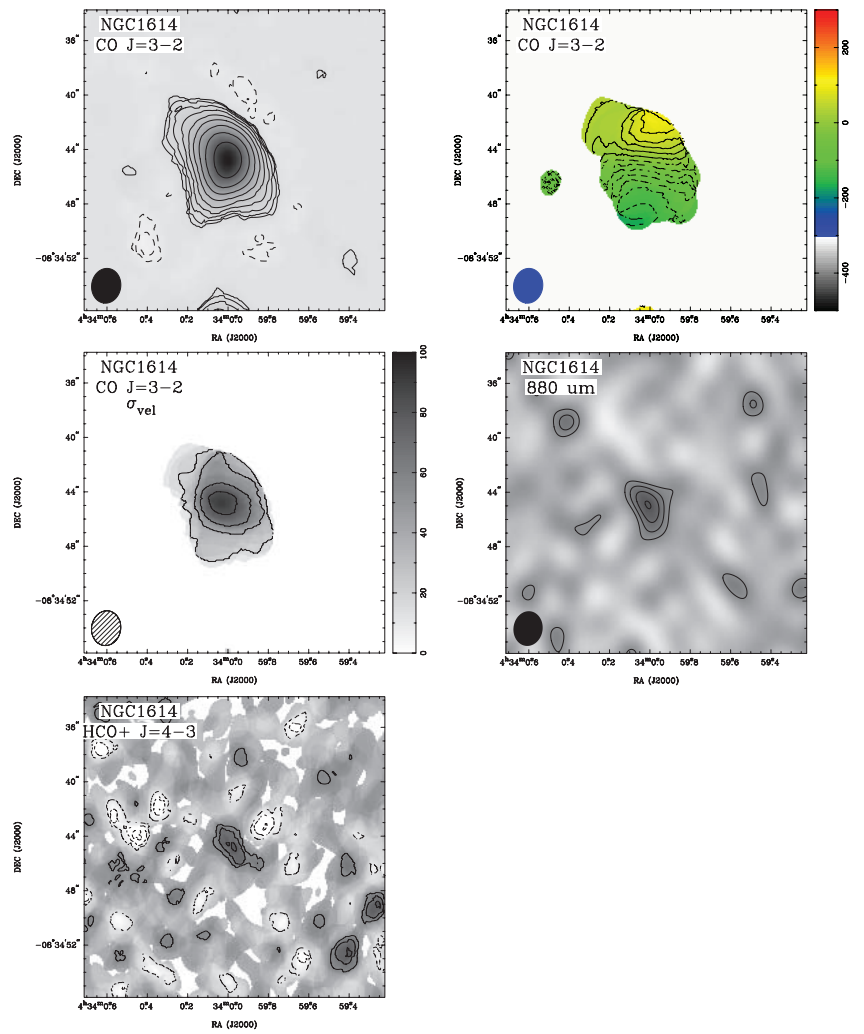

FIG. 23.-NGC $1614 \mathrm{CO} J=3-2$ and $880 \mu \mathrm{m}$ continuum maps. Notation as in Figs. 2 and 3. Top left: COJ $=3-2$ moment 0 map. Lowest contour is $\pm 2 \sigma=$ $8.2 \mathrm{Jy} \mathrm{beam}^{-1} \mathrm{~km} \mathrm{~s}^{-1}$ and contours increase by factors of 1.5. Top right: $\mathrm{CO}$ $J=3-2$ moment 1 map. Contours are $20 \mathrm{~km} \mathrm{~s}^{-1} \times(-8,-7,-6,-5,-4,-3$, $-2,-1,0,1,2,3,4,5)$ relative to $c z$. Middle left: $\mathrm{CO} J=3-2$ moment 2 map. Contours are $20 \mathrm{~km} \mathrm{~s}^{-1} \times(1,2,3,4)$. Middle right: Uncleaned $880 \mu \mathrm{m}$ map. Lowest contour is $2 \sigma=8.2 \mathrm{mJy}$, and contours increase in steps of $1 \sigma$. Bottom left: Uncleaned $\mathrm{HCO}^{+} J=4-3$ moment 0 map. Lowest contour is $\pm 2 \sigma=$ $6.24 \mathrm{Jy} \mathrm{beam}^{-1} \mathrm{~km} \mathrm{~s}^{-1}$, and contours increase in steps of $1 \sigma$.
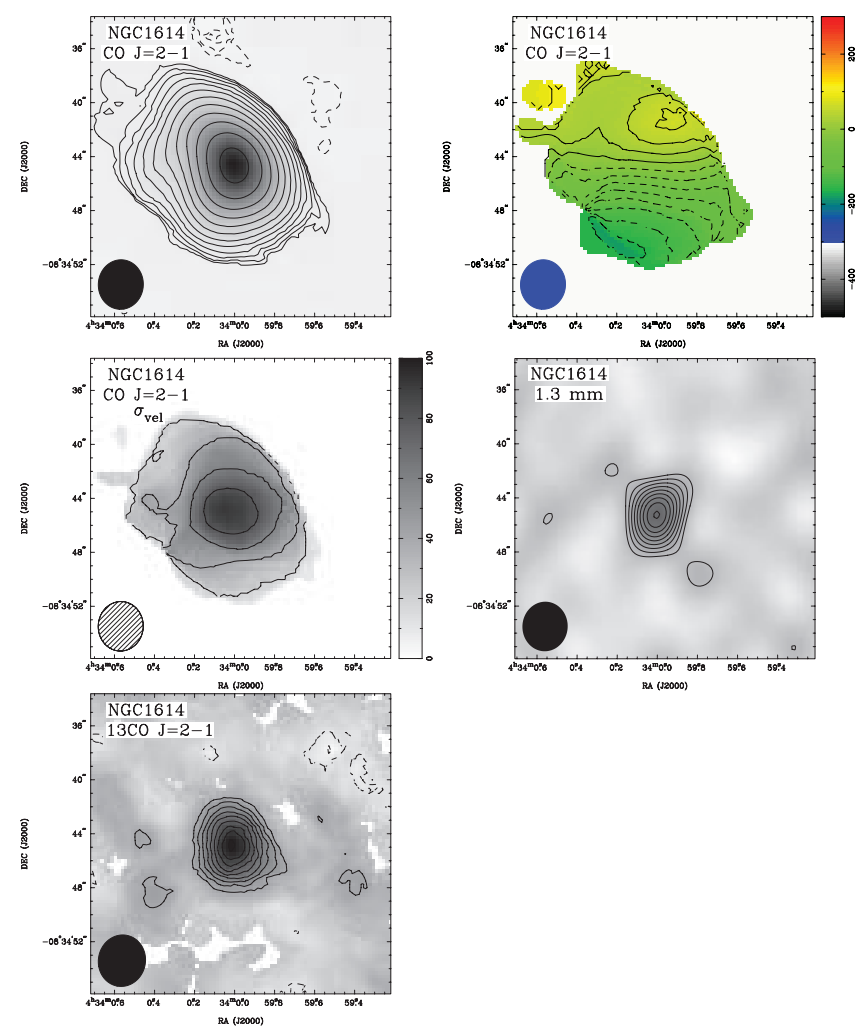

FIG. 24.-NGC $1614 \mathrm{CO} J=2-1$ and $1.3 \mathrm{~mm}$ continuum maps. Notation as in Fig. 2 and 10. Top left: $\mathrm{CO} J=2-1$ moment 0 map. Lowest contour is $\pm 2 \sigma=$ $3.0 \mathrm{Jy}$ beam $^{-1} \mathrm{~km} \mathrm{~s}^{-1}$, and contours increase by factors of 1.5. Top right: CO $J=2-1$ moment 1 map. Contours are $20 \mathrm{~km} \mathrm{~s}^{-1} \times(-8,-7,-6,-5,-4,-3$, $-2,-1,0,1,2,3,4,5)$ relative to $c z$. Middle left: $\mathrm{CO} J=2-1$ moment 2 map. Contours are $20 \mathrm{~km} \mathrm{~s}^{-1} \times(1,2,3,4)$. Middle right: Uncleaned $1.3 \mathrm{~mm}$ map. Lowest contour is $2 \sigma=2.6 \mathrm{mJy}$, and contours increase in steps of $1 \sigma$. Bottom left: ${ }^{13} \mathrm{CO}$ $J=2-1$ moment 0 map. Lowest contour is $\pm 2 \sigma=2.5 \mathrm{Jy} \mathrm{beam}^{-1} \mathrm{~km} \mathrm{~s}^{-1}$, and contours increase in steps of $1 \sigma$. 

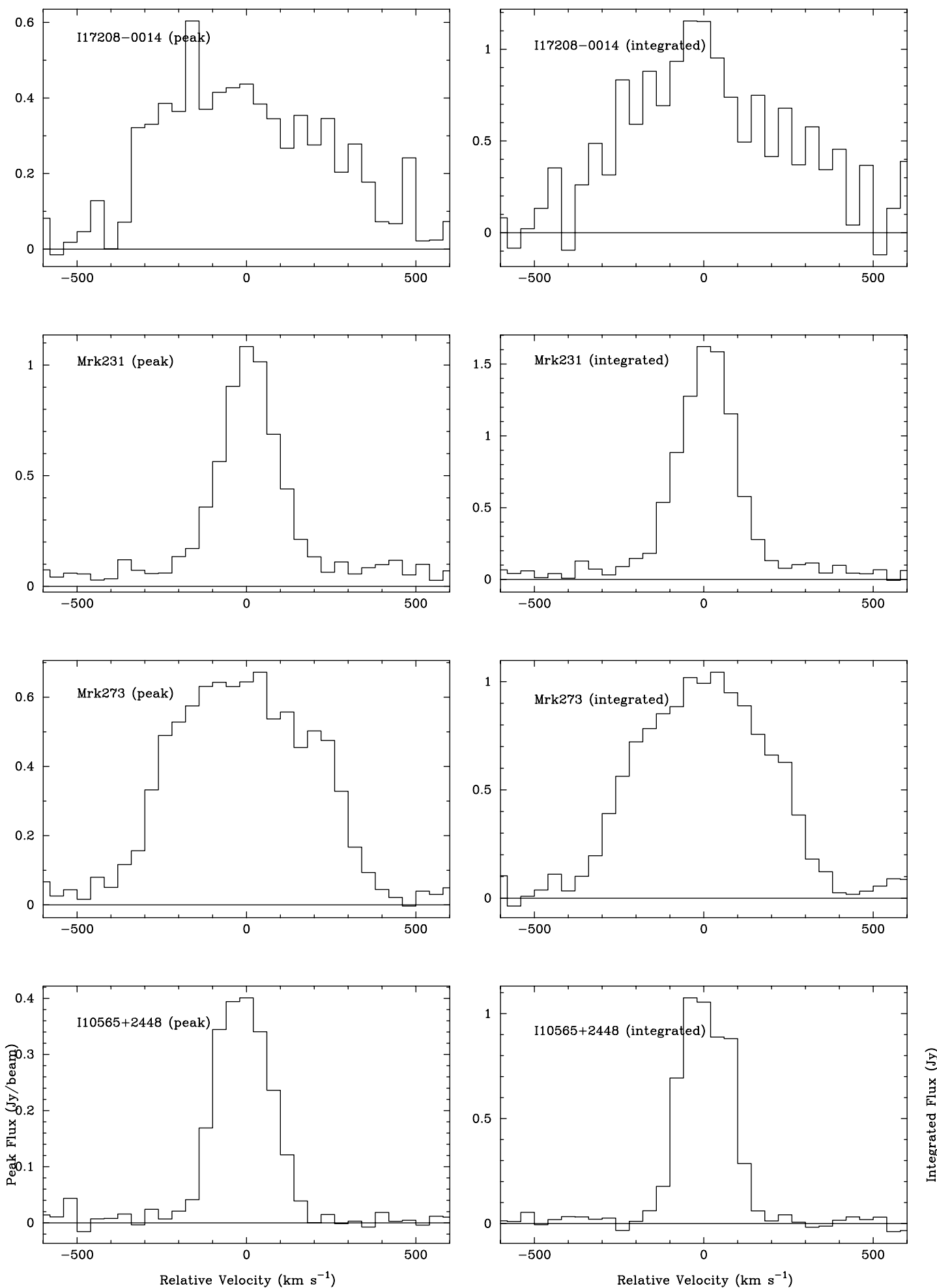

FIG. 25.- Peak and integrated spectra of the ${ }^{12} \mathrm{CO} J=3-2$ emission for four U/LIRGs from our sample. The integrated spectrum is the sum of the emission inside a rectangular region whose size was chosen to encompass all of the emission above $2 \sigma$ in the moment 0 map. The velocity scale is relative to the recession velocity for each galaxy given in Table 1. 

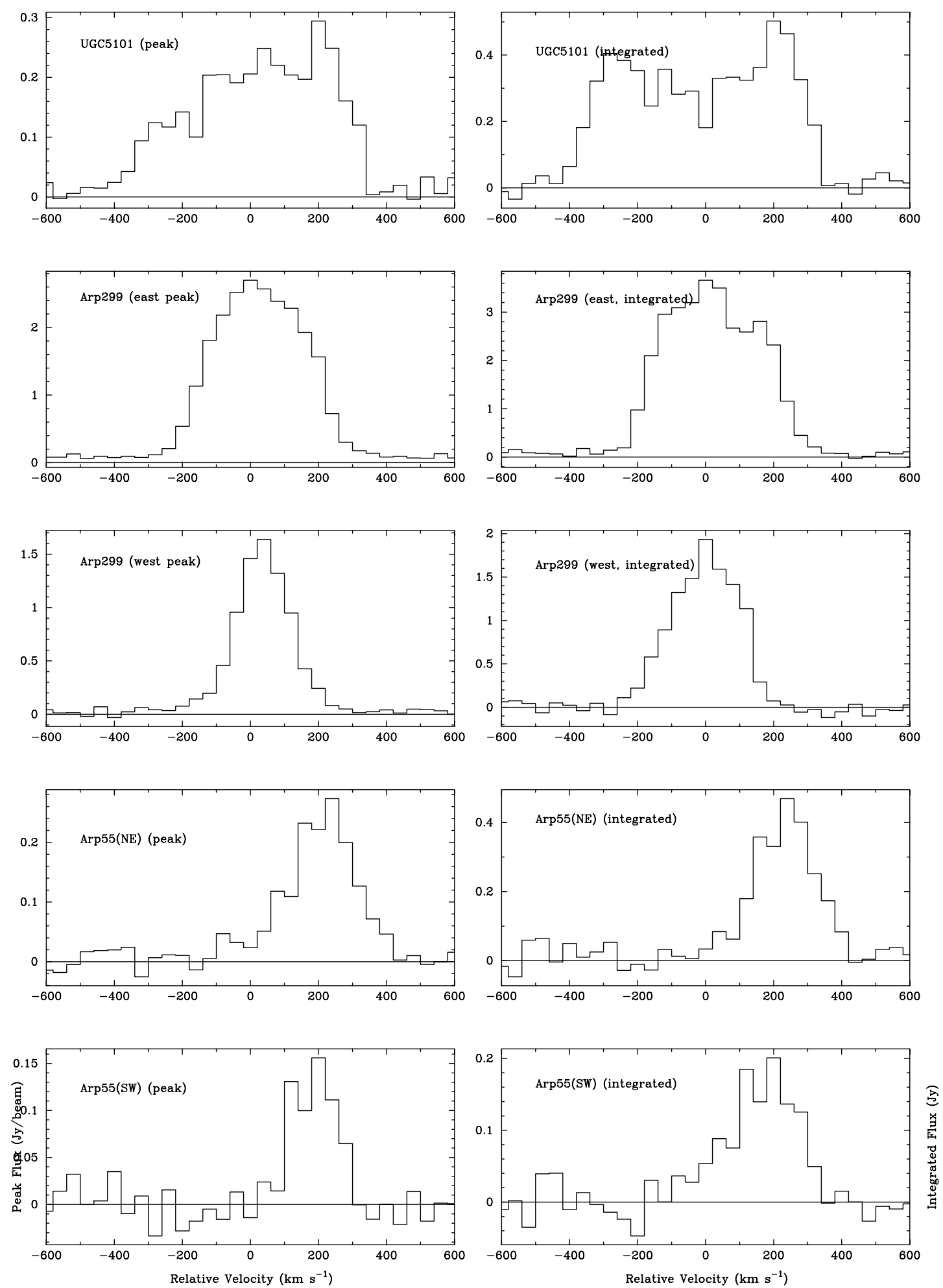

FIG. 26.- Peak and integrated spectra of the ${ }^{12} \mathrm{CO} J=3-2$ emission for three U/LIRGs from our sample. See Fig. 25 for additional details. 

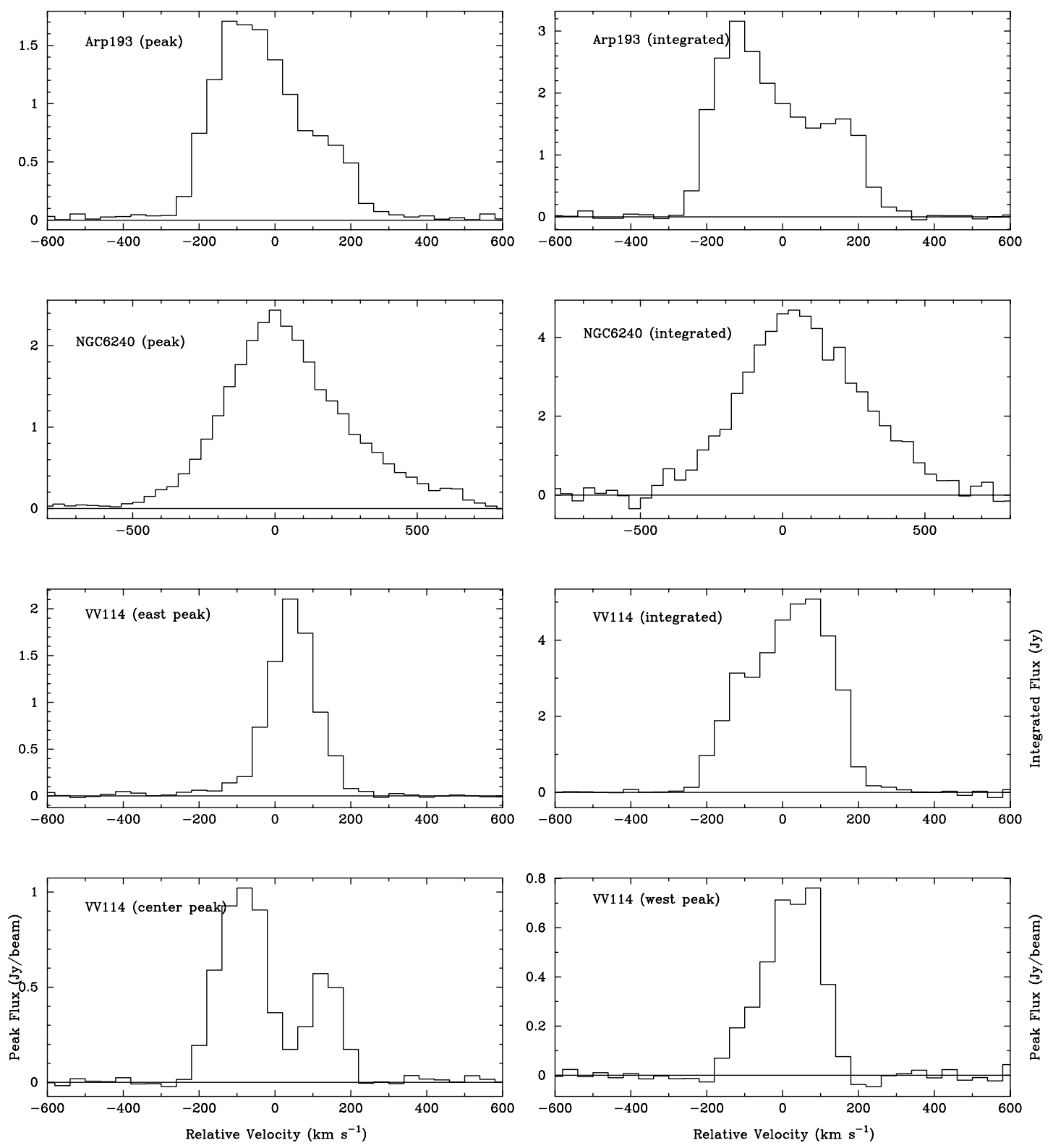

FIG. 27.- Peak and integrated spectra of the ${ }^{12} \mathrm{CO} J=3-2$ emission for three U/LIRGs from our sample. Note the wider velocity scale used for NGC 6240 . See Fig. 25 for additional details.

single component fits $(\S 3.1)$, this would have a net effect of decreasing the gas-to-dust mass ratio.

Another source of uncertainty comes from the value of the dust emissivity that we have assumed. A value of $\kappa=0.9 \mathrm{~cm}^{2} \mathrm{~g}^{-1}$ is appropriate for moderately dense gas in star-forming regions in the Galaxy (Henning et al. 1995; Johnstone et al. 2000), while Dunne et al. (2000) assumed $\kappa=0.77 \mathrm{~cm}^{2} \mathrm{~g}^{-1}$ (Draine \& Lee 1984; Hughes et al. 1993) in deriving dust masses for their survey of nearby galaxies. It is possible that the dust emissivity is modified drastically in the hot, dense central regions of these galaxies. However, without sufficient data at high angular resolution to fit physical dust models (see below), it is impossible at the moment to determine how large an effect this might be. In any case, the dust masses determined here are likely uncertain by a factor of less than 2 due to the uncertain value of $\kappa$.

The ideal procedure for determining dust mass would be to fit a detailed physical model to the full infrared spectral energy distribution, as has been done by Galliano et al. (2003) for the dwarf galaxy NGC 1569 or Regan et al. (2004) for NGC 7331. However, only near-infrared and ground-based mid-infrared data can approach the angular resolution of our $880 \mu \mathrm{m}$ SMA data. The critical mid- and far-infrared spectral regions can be covered by the Spitzer Space Telescope, for which the angular resolution ranges from $1.5^{\prime \prime}$ at $3.6 \mu \mathrm{m}$ to $47^{\prime \prime}$ at $160 \mu \mathrm{m}$. Near-infrared data from Spitzer can probe the hot dust continuum (Higdon et al. 2006), while data for the mid- and far-infrared part of the spectrum only allow a determination of galaxy-wide dust properties and do not isolate the central regions discussed here. In addition, diffraction-limited groundbased mid-infrared photometry at $12.5 \mu \mathrm{m}$ is available for nine galaxies in our sample (Soifer et al. 2000, 2001); however, no data beyond $30 \mu \mathrm{m}$ with arcsecond-scale resolution are currently available from any instrument. We defer a more detailed analysis of the dust temperature and properties inferred by combining our submillimeter data with infrared data to a future paper.

\subsection{Total Gas and Dust Masses from SMA Data}

The central $\mathrm{H}_{2}$ gas masses calculated from the total luminosities detected with the SMA range from $1.4 \times 10^{9} M_{\odot}$ for NGC 5257 

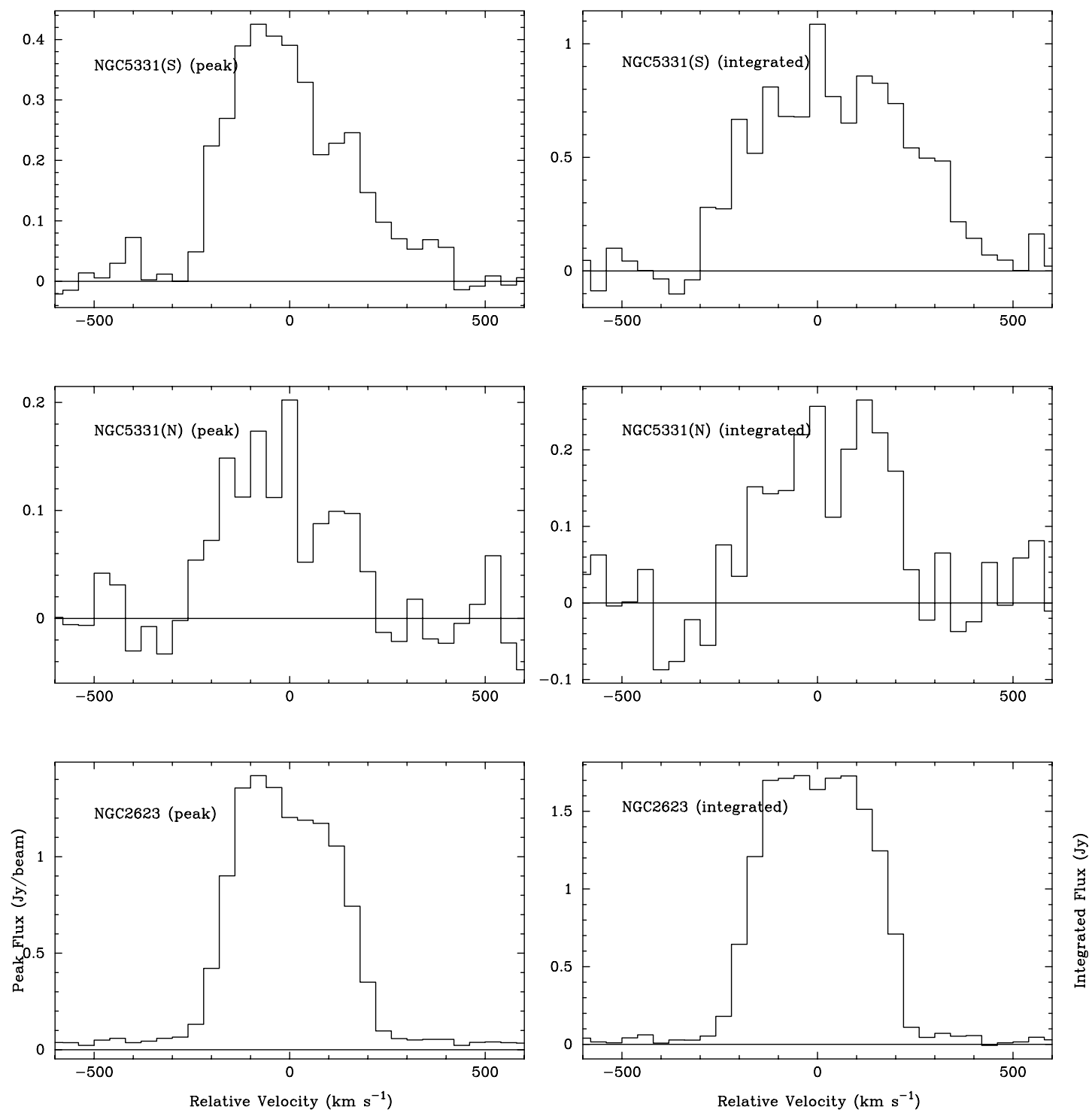

FIG. 28. - Peak and integrated spectra of the ${ }^{12} \mathrm{CO} J=3-2$ emission for two U/LIRGs from our sample. See Fig. 25 for additional details.

to $1.2 \times 10^{10} M_{\odot}$ for NGC 6240 while the central dust masses range from $3 \times 10^{6} M_{\odot}$ for NGC 1614 to $8 \times 10^{7} M_{\odot}$ for Mrk 231 (Table 6). The central gas-to-dust ratios also show a wide variation, from as low as 29 for Arp 55(NE) to as high as 720 for NGC 6240.

Excluding the three galaxy components with nondetections in the continuum, the average gas-to-dust ratio is $215 \pm 53$ (rms deviation 207). This value is about $40 \%$ larger than the typical Milky Way value of $\sim 150$ (Draine et al. 2007), and there is a large dispersion of the individual values about the average. Our value is also significantly smaller than the value of 540 (rms deviation 290) determined by Sanders et al. (1991) using single-dish CO $J=1-0$ data and far-infrared luminosities. However, that study adopted a CO-to- $\mathrm{H}_{2}$ conversion factor that is 6 times larger than our value, which almost certainly results in an overestimate of the total amount of molecular gas in these galaxies. Adopting the same conversion factor as used in this paper would give a gas-to-dust ratio of 90 for the data in Sanders et al. (1991) somewhat smaller than the ratio obtained here.

The extremely low values seen for Arp 55 and, to some extent, for UGC 5101 and Mrk 231 may be due to a significant contribution to the $880 \mu \mathrm{m}$ flux from nonthermal or radio free-free emission. However, Arp 55(NE) shows no evidence for a central AGN that could be a source of nonthermal emission (see the Appendix). There is some evidence to suggest that UGC 5101 contains a buried AGN (see the Appendix), which could increase the $880 \mu \mathrm{m}$ flux and lead to a small overestimate in the dust mass and an underestimate of the gas-to-dust ratio. Mrk 231 is the one galaxy in our sample for which a dominant AGN contribution has been identified previously at millimeter wavelengths (Downes \& Solomon 1998); it is possible that we have underestimated the contribution from the AGN at $880 \mu \mathrm{m}$ in our calculations. Mrk 273, Arp 299, NGC 6240, and NGC 2623 have all been identified as containing an AGN from X-ray data (Xia et al. 2002; Zezas et al. 2003; Komossa et al. 2003; Maiolino et al. 2003); however, the contribution of nonthermal emission from the AGN at millimeter wavelengths appears to be small (see the Appendix).

Extremely high values for the gas-to-dust ratio are measured for NGC 6240, VV 114, and NGC 1614. For NGC 6240, the very broad $\mathrm{CO}$ emission line reduces the bandwidth available to measure the continuum flux and produces a large uncertainty in the flux. In addition, the $\mathrm{CO} J=3-2$ emission may be moderately optically thin (Draine et al. 2007), which could result in the gas mass being overestimated. VV 114 and NGC 1614 are some 

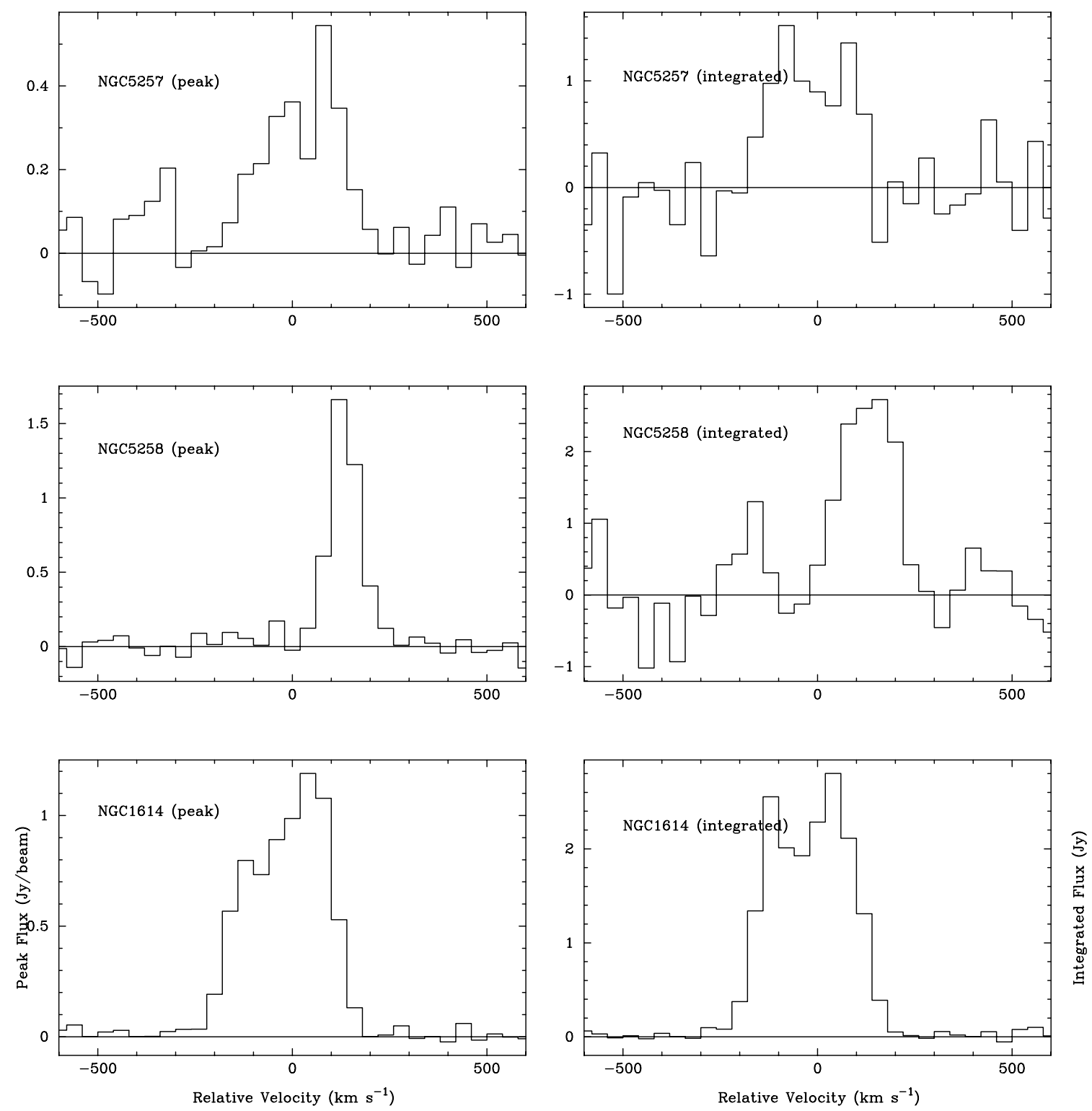

FIG. 29.- Peak and integrated spectra of the ${ }^{12} \mathrm{CO} J=3-2$ emission for two U/LIRGs from our sample. See Fig. 25 for additional details.

of the nearest galaxies in our sample and, along with Arp 299, are the only ones for which large amounts of missing flux produce negative bowls in the $\mathrm{CO} J=3-2$ maps. Because these galaxies are relatively nearby, the SMA has greater sensitivity to low surface density gas that is likely to be spatially extended than it would have in some of our more distant galaxies. Figures 15 and 23 show that the $\mathrm{CO}$-emitting regions in these galaxies are quite extended; however, the dust continuum emission does not appear to be significantly extended in these three galaxies. The compact nature of the dust emission suggests that the SMA data are not sufficiently sensitive to pick up continuum emission from dust outside the peak central concentration. In addition, the continuum data suffer slightly more from the missing flux problem than the spectral-line data do ( $§ 2.4$ and Tables 4 and 5), because the line emission is intrinsically more compact in an individual spectral channel.

An examination of the relative strengths of the $\mathrm{CO} J=3-$ 2 emission and the $880 \mu \mathrm{m}$ emission suggests that this is a reasonable explanation. For example, if the gas-to-dust mass ratio were 150 , then, using the equations for dust and gas masses given above, we would expect the flux in the $\operatorname{CO} J=3-2$ moment map to be roughly $10^{4}$ times brighter (in $\mathrm{Jy} \mathrm{km} \mathrm{s}^{-1}$ ) than the flux in the $880 \mu \mathrm{m}$ map (in janskys). For NGC 1614 and Arp 299, if we compare the two maps using contours scaled by this factor of $10^{4}$, we see a very similar extent and relative strength in the $\mathrm{CO}$ $J=3-2$ and $880 \mu \mathrm{m}$ maps. For VV 114, the central and western peaks are similar in extent and relative strength in the CO $J=$ 3-2 and $880 \mu \mathrm{m}$ maps. However, the eastern peak is fainter in $880 \mu \mathrm{m}$ continuum than we would expect by a factor of about 2 , which suggests that either the gas-to-dust ratio or some other property of the gas and dust (such as CO emissivity or temperature) is different in this region.

\subsection{Gas and Dust Masses in the Central Kiloparsec}

The previous discussion combined with the large range in the gas-to-dust mass ratios seen in Table 6 led us to examine the gasto-dust ratio using a single resolution element to probe the most central region of each galaxy or galaxy component. For these calculations, we compared the $\mathrm{H}_{2}$ mass calculated from the integrated $\mathrm{CO} J=3-2$ intensity in a single beam at the peak of the 

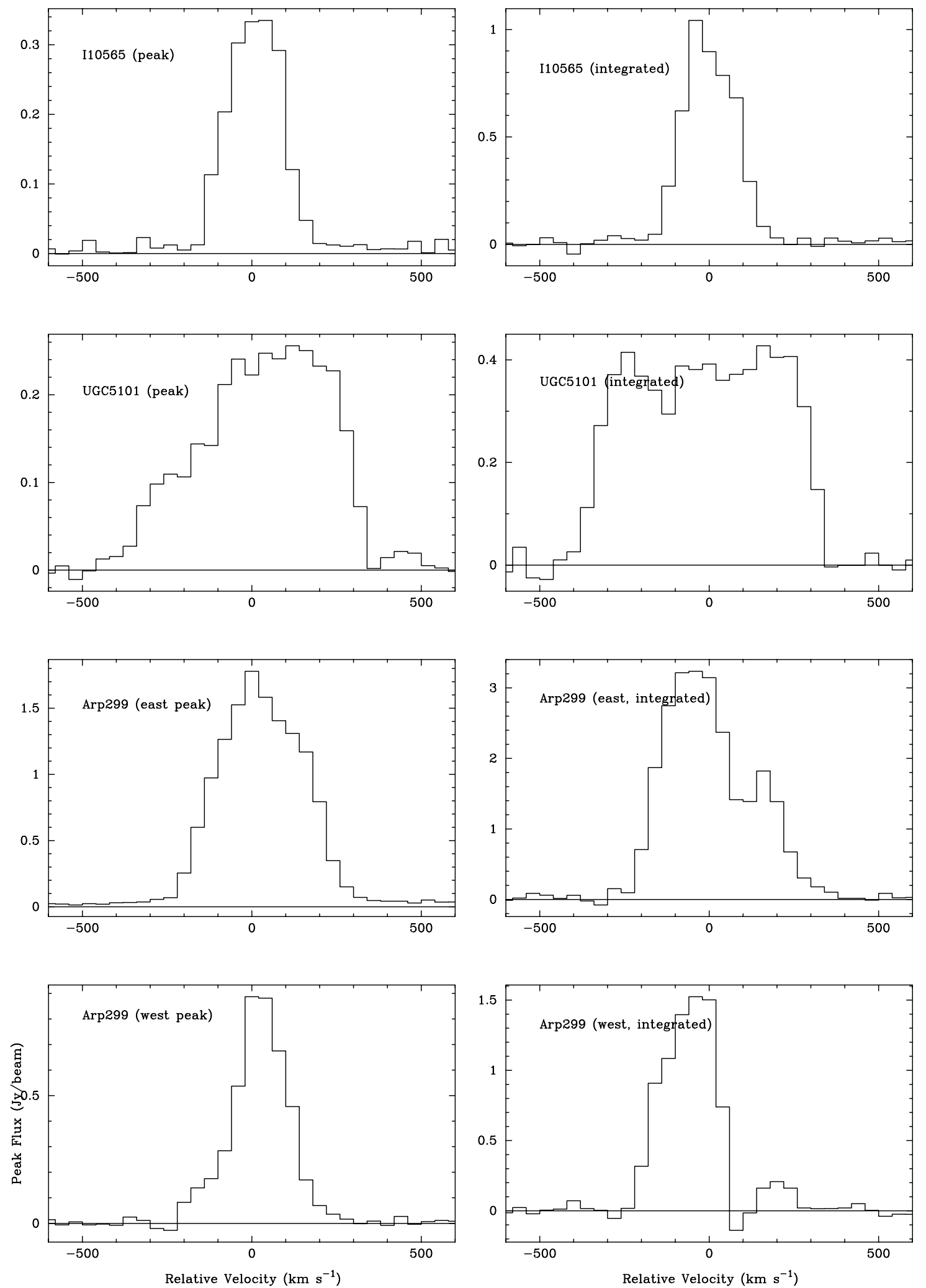

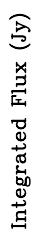

FIG. 30.-Peak and integrated spectra of the ${ }^{12} \mathrm{CO} J=2-1$ emission for three U/LIRGs from our sample. See Fig. 25 for additional details. 

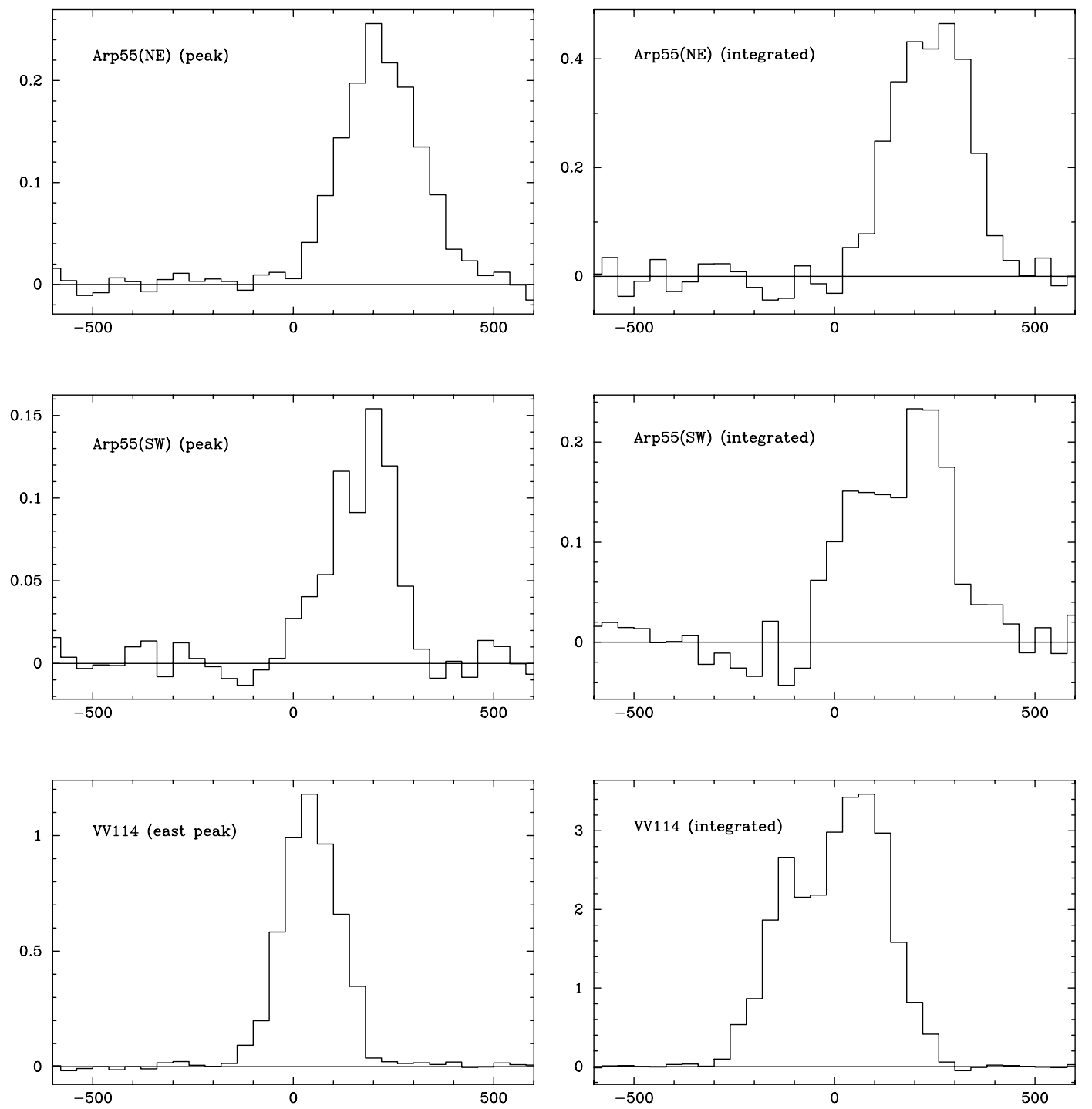

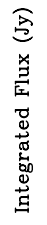
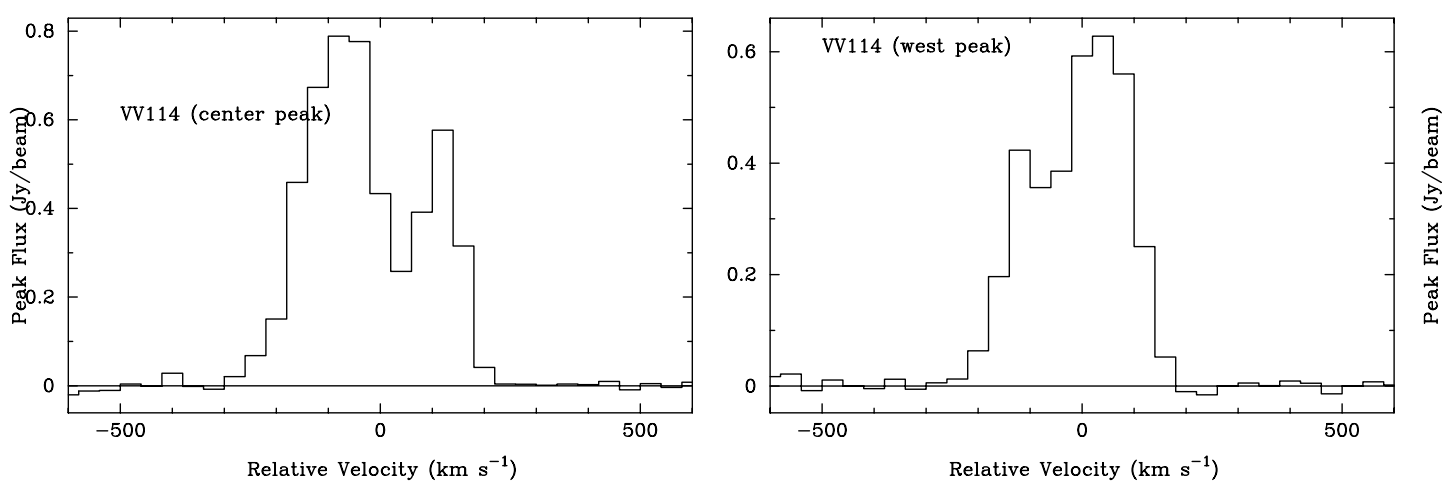

FIG. 31.-Peak and integrated spectra of the ${ }^{12} \mathrm{CO} J=2-1$ emission for two U/LIRGs from our sample. The integrated spectrum for VV 114 encompasses the entire extended emission region. See Fig. 25 for additional details. 

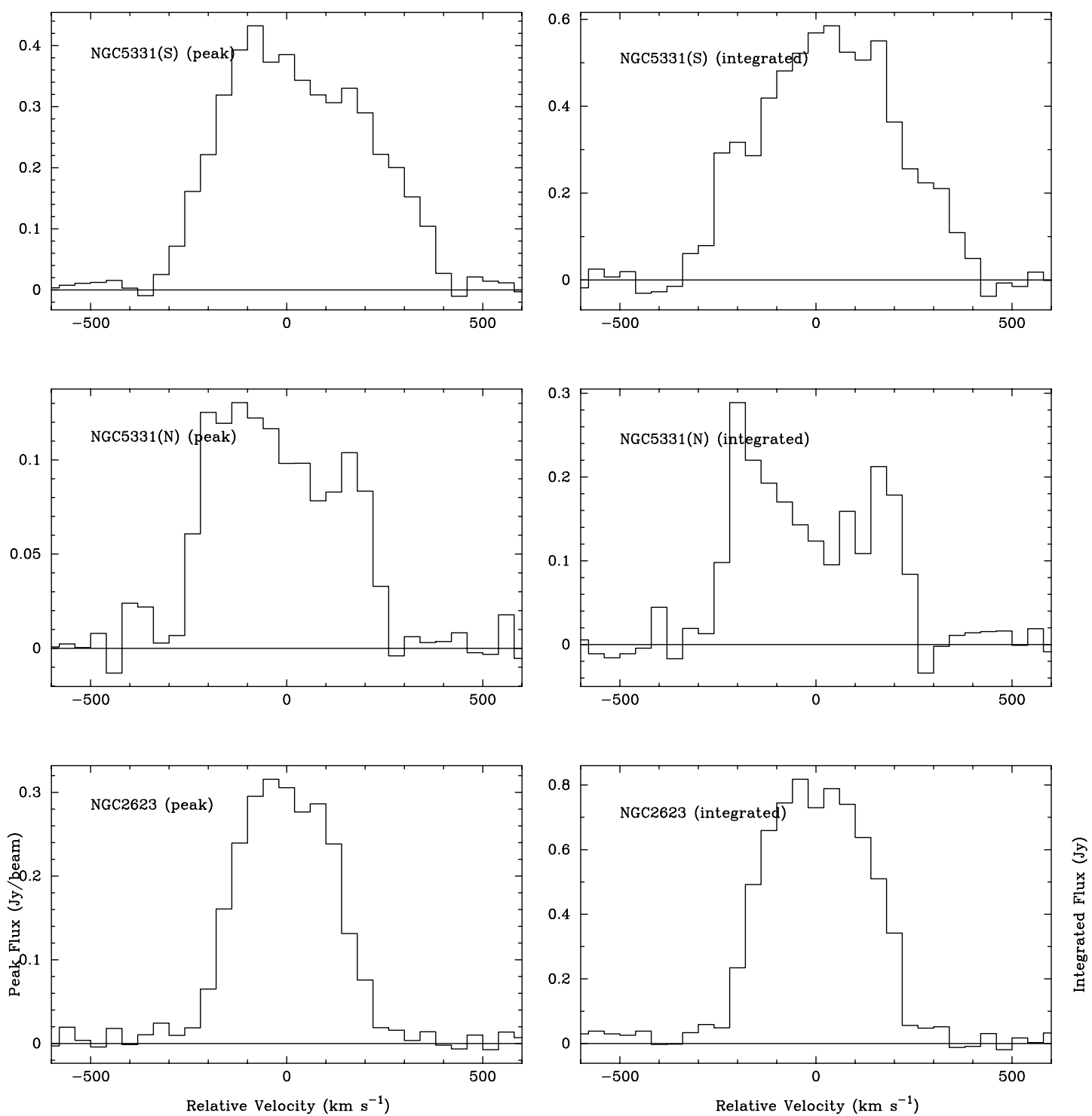

FIG. 32.- Peak and integrated spectra of the ${ }^{12} \mathrm{CO} J=2-1$ emission for two U/LIRGs from our sample. See Fig. 25 for additional details.

emission with the peak $880 \mu \mathrm{m}$ continuum intensity (Table 7). Note that since many of the continuum sources are not resolved, the peak continuum emission given in Table 7 has the same value as the integrated flux given in Table 3 .

The average gas-to-dust ratio including all 15 galaxies or galaxy components with continuum detections in Table 7 is $120 \pm$ 28 (rms deviation 109). This value is smaller than the average value obtained from the values in Table 6 (i.e., using the total mass detected with the SMA data) and agrees somewhat better with the typical Milky Way gas-to-dust ratio. Perhaps more importantly, the dispersion about the mean value is smaller by about a factor of 2 when only the peak values are used, which suggests some of the dispersion seen in the integrated values can be attributed to the better mass sensitivity of the CO observations (see $\S 3.3$ ).

Thus, our new SMA data suggest that the gas-to-dust mass ratio in the central kiloparsec of these LIRGs and ULIRGs is very similar to the gas-to-dust ratio measured in the Milky Way. This result is somewhat surprising given that the dust in these nuclear regions is subject to more intense heating (as well as perhaps processing due to shocks) compared to typical regions in the Milky Way. Perhaps the main zone of activity is very compact and so does not dominate the energetics of the interstellar medium in the entire central kiloparsec; this could be tested by subarcsec resolution observations in a direct star formation tracer. Alternatively, perhaps most of the gas and dust reside in high column density clouds or cores that help to shield much of the mass from external heating.

\subsection{Possible Variations in Gas and Dust Properties}

The normal gas-to-dust ratio seen in these galaxies provides additional validation for the $\mathrm{CO}-$ to- $\mathrm{H}_{2}$ conversion factor derived by Downes \& Solomon (1998) and adopted in this analysis. In this section, we check for any systematic differences in the gasto-dust ratio among the galaxies in our sample that may point to differences in the interstellar medium properties. Our overall picture is one in which the formation and heating of dust tracks the location of molecular gas, regardless of whether that gas is distributed largely in discrete virialized clouds (as in the Galaxy) or to a large extent in a diffuse intracloud medium (as in ULIRGs, where the $\mathrm{CO}-$ to- $\mathrm{H}_{2}$ conversion factor is consequently lower). We might expect there to be some differences in our sample, which spans a factor of 10 in infrared luminosity and exhibits nuclear separations from less than $1 \mathrm{kpc}$ (if multiple nuclei are 

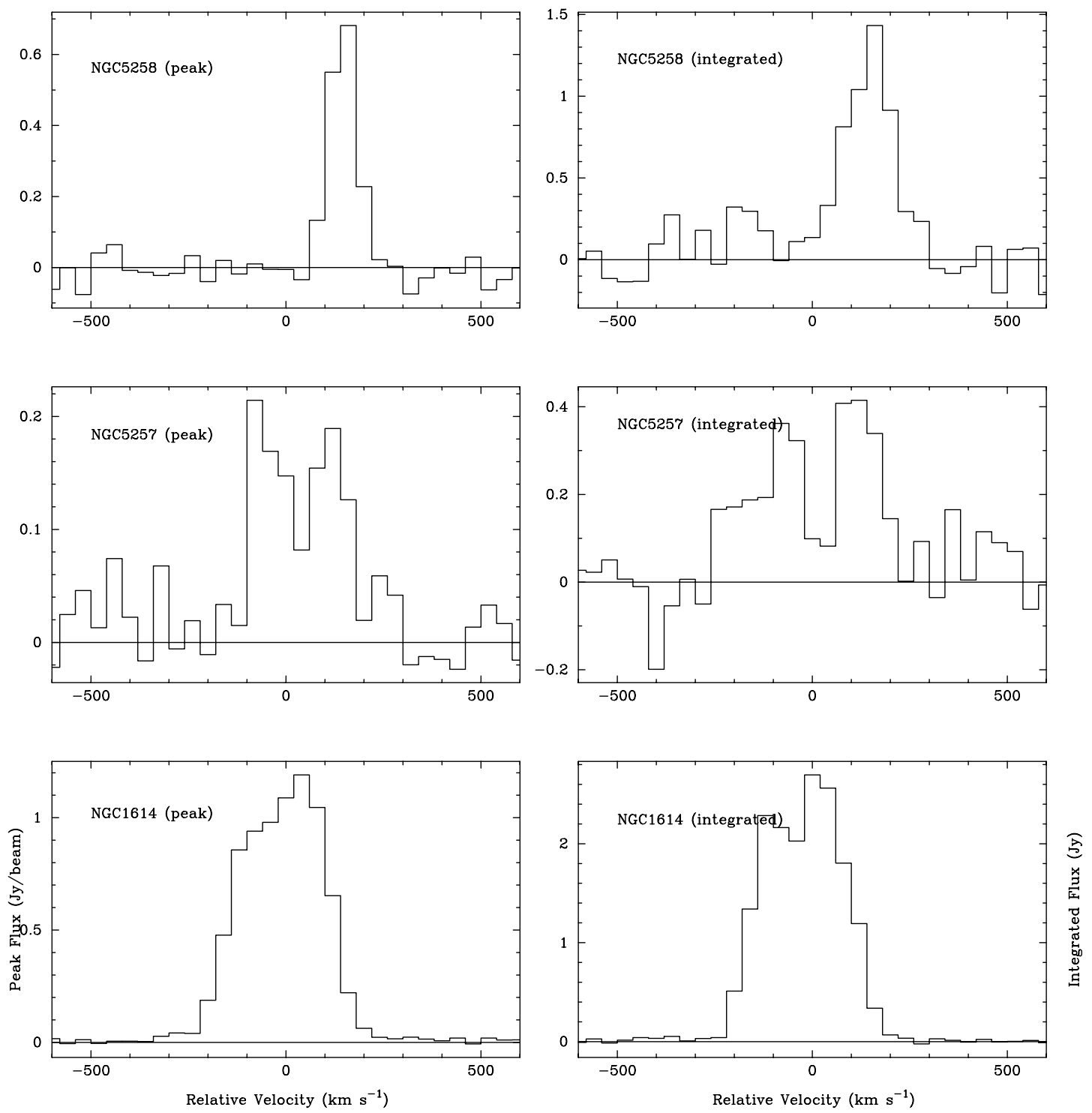

FIG. 33.- Peak and integrated spectra of the ${ }^{12} \mathrm{CO} J=2-1$ emission for two U/LIRGs from our sample. See Fig. 25 for additional details.

seen at all) to $20 \mathrm{kpc}$. Relatively undisturbed galaxies with a larger nuclear separation are more likely to have a normal interstellar medium with a more normal $\mathrm{CO}-$ to- $\mathrm{H}_{2}$ conversion factor, which would increase the gas mass and the gas-to-dust ratio.

Among the 14 objects in our sample are five systems in which the two progenitor galaxies can still be clearly distinguished (Arp 299, Arp 55, VV 114, NGC 5331, and NGC 5257/8), while the remaining nine objects show a single nucleus or a pair of nuclei separated by less than $1 \mathrm{kpc}$. If we calculate the gas-to-dust ratio in the central kiloparsec for the extended and the compact galaxies separately, we find no significant difference between the two samples. If we divide the sample as a function of luminosity, we find that the average gas-to-dust ratio calculated for the lower luminosity galaxies (160-180 depending on where we divide the sample) is roughly 3 times larger than the gas-to-dust ratio for the more luminous galaxies $(50-70)$. Thus, there appears to be a strong dependence of the central gas-to-dust ratio on infrared luminosity in our sample, but not on nuclear separation.

These nominally discrepant ratios are in fact compatible with a reasonably uniform gas-to-dust ratio of $\sim 100-150$ once plausible corrections are made. We consider here simply changes to the $\mathrm{CO}-$ to- $\mathrm{H}_{2}$ conversion factor, how the dust temperature is derived (optically thick vs. optically thin), and the origin of the submillimeter luminosity. We do not consider varying the absolute value of the dust emissivity, $\kappa$, since this is relatively poorly constrained in any case. Since the less luminous galaxies show on average good agreement with the Milky Way's gas-to-dust ratio, we focus on possible changes to our analysis that could increase the gas-to-dust ratio in the more luminous galaxies.

One possibility is that the dust temperature is underestimated by our optically thin models and that in fact a higher dust temperature, such as those derived by Solomon et al. (1997) is more appropriate. Using the optically thick dust temperatures would raise the gas-to-dust ratio in these galaxies by about a factor of 2 . A second possibility is that there is significant nonthermal or free-free emission contaminating the $880 \mu \mathrm{m}$ flux. This is difficult to quantify but would also act to raise the gas-to-dust ratio. There can be a significant contribution to the far-infrared luminosity from a buried AGN, and this contribution can increase with increasing $L_{\mathrm{FIR}}$ (Tran et al. 2001). Assuming that the AGN also contributes significant emission at $880 \mu \mathrm{m}$, this effect has the right sense to explain the trend of the gas-to-dust ratio with $L_{\mathrm{FIR}}$. In contrast, adopting a larger value for the CO-to- $\mathrm{H}_{2}$ conversion factor (to bring it closer to the value in normal spiral galaxies) 

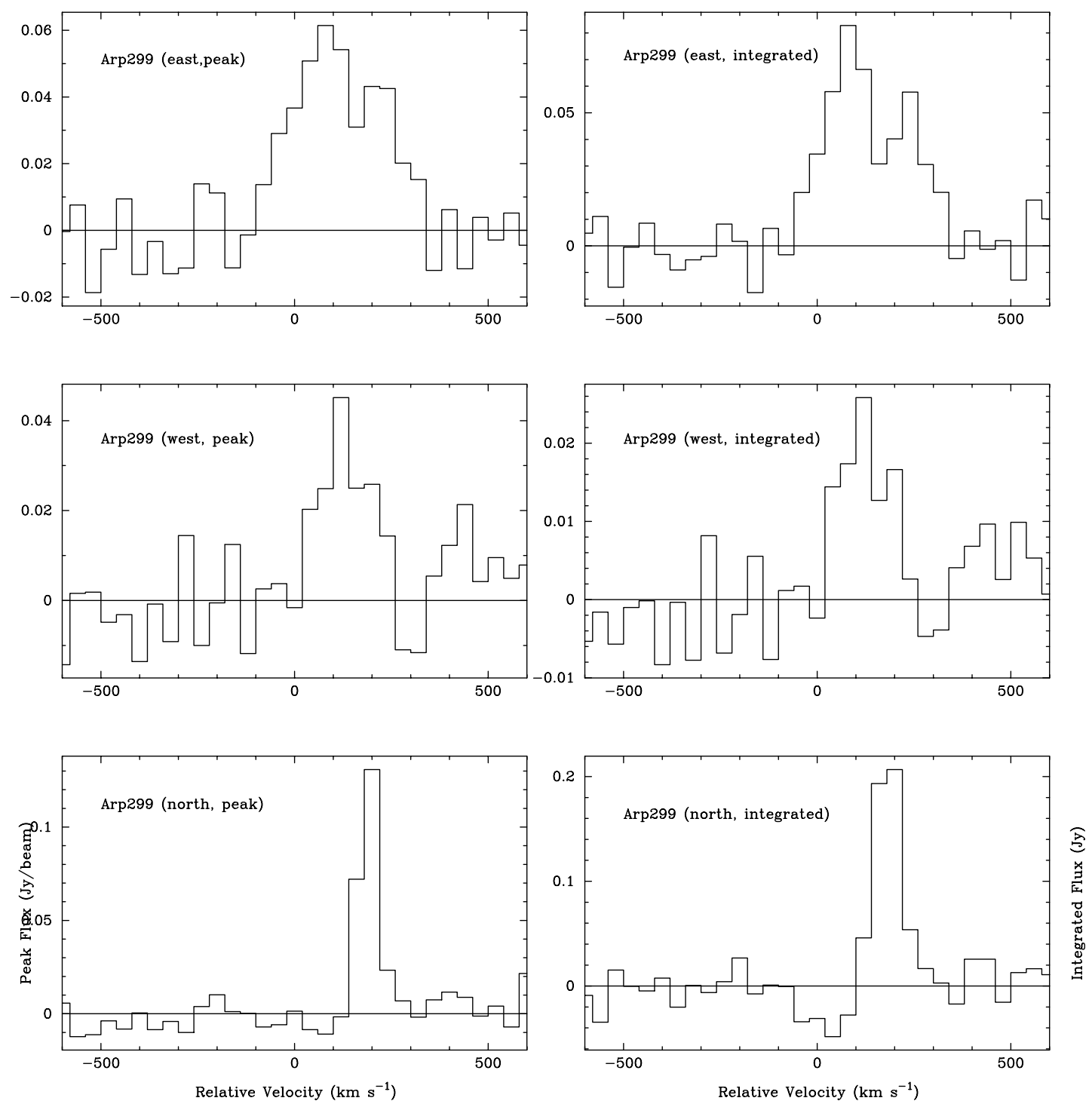

Fig. 34.-Peak and integrated spectra of the ${ }^{13} \mathrm{CO} J=2-1$ emission for three regions in the interacting galaxy Arp 299 for which ${ }^{13} \mathrm{CO} J=2-1$ emission was detected. See Fig. 25 for additional details.

would also increase the gas-to-dust ratio, but would not be consistent with previous studies of these extremely luminous galaxies.

\section{CORRELATIONS BETWEEN GAS AND DUST PROPERTIES IN THE SAMPLE}

\subsection{Testing for Correlations}

We have searched for correlations among the gas and dust properties in the galaxies in our sample. The physical and observational quantities that were considered were far-infrared luminosity, dust temperature, the peak $\mathrm{H}_{2}$ surface density derived from the $\mathrm{CO} J=3-2$ emission, $\mathrm{CO} J=3-2$ beam area, total $\mathrm{H}_{2}$ gas mass derived from the $\mathrm{CO} J=3-2$ emission detected with the SMA, the ratio of the far-infrared luminosity to the total $\mathrm{H}_{2}$ mass, the ratio of the far-infrared luminosity to the peak $\mathrm{H}_{2}$ mass, the peak gas-to-dust mass ratio, and the CO $J=3-2 / 2-1$ line ratio measured from the total flux detected with the SMA. This list includes both physical quantities associated with the star formation and interstellar medium properties as well as properties of the sample (such as beam area and distance) that may introduce selection effects. We note that the $\mathrm{CO} J=3-2 / 2-1$ line ratio could be underestimated if the SMA maps resolve out a larger fraction of the flux in the higher frequency line. We also note that the peak $\mathrm{H}_{2}$ surface density may be underestimated in IRAS 17208-0014, which has a bright unresolved core and also somewhat underestimated in six galaxies (Mrk 231, Mrk 273, IC 694 [Arp 299], Arp 55 NE, Arp 193, and NGC 2623) for which the $\mathrm{CO}$ emission is resolved along the long axis but only slightly extended relative to the beam along the short axis of emission.We calculated the correlations using the method described in Akritas \& Siebert (1996) to correct for any distance biases in our sample.

We identified 11 correlations that are statistically significant at the $95 \%$ confidence level or better ( $p \leq 0.05$, where $p$ is the probability of a false correlation after removing the effect of distance; Table 8): far-infrared luminosity and peak $\mathrm{H}_{2}$ surface density, far-infrared luminosity and dust temperature, dust temperature and peak $\mathrm{H}_{2}$ surface density, beam area and peak $\mathrm{H}_{2}$ surface density, $\mathrm{CO} J=3-2 / 2-1$ line ratio and beam area, $\mathrm{CO}$ $J=3-2 / 2-1$ line ratio and total $\mathrm{H}_{2}$ mass detected with the SMA, and the ratio of the far-infrared luminosity to the total $\mathrm{H}_{2}$ mass detected with the SMA with each of far-infrared luminosity, dust 

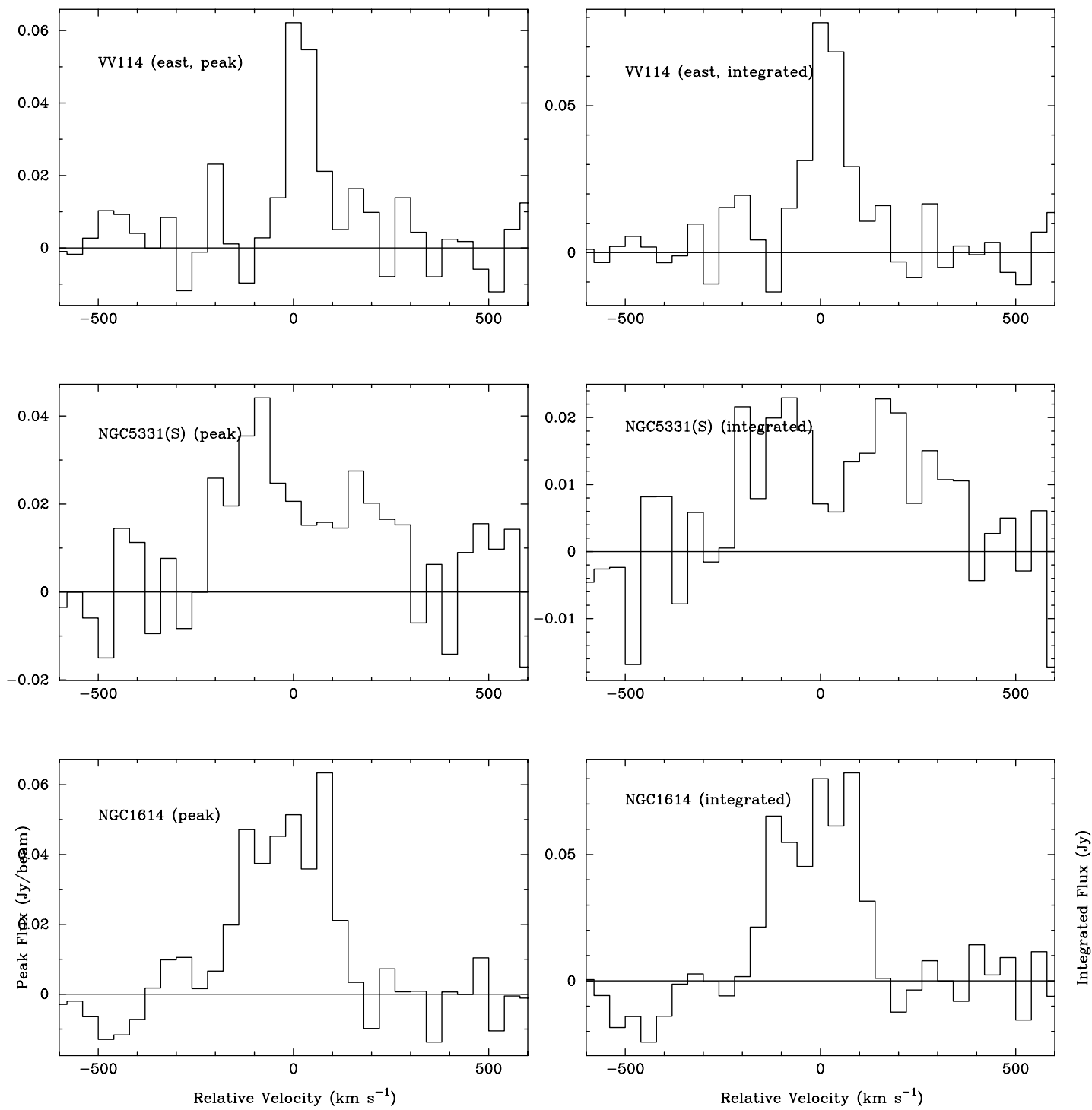

FIG. 35.-Peak and integrated spectra of the ${ }^{13} \mathrm{CO} J=2-1$ emission for three U/LIRGs from our sample for which ${ }^{13} \mathrm{CO} J=2-1$ emission was detected. See Fig. 25 for additional details.

temperature, beam area, $\mathrm{CO} J=3-2 / 2-1$ line ratio, and the ratio of the far-infrared luminosity to the peak $\mathrm{H}_{2}$ mass.

However, the correlation of surface density with beam area becomes consistent with a null correlation if we use only the 12 galaxies for which the linear resolution of the beam lies between 0.5 and $1.1 \mathrm{kpc}$, e.g., excluding NGC 5257 and Arp 299. In addition, all five of the correlations with the ratio of the far-infrared luminosity to the total $\mathrm{H}_{2}$ mass become consistent with a null correlation if we remove NGC 5257 or Arp 299 from the data set. Thus, we conclude that these five correlations are less robust. The remaining five correlations are all significant at the $99 \%$ confidence level except for far-infrared luminosity with dust temperature, which is significant at the $98 \%$ confidence level; however, this correlation is well known from previous studies with larger samples (Soifer et al. 1989). Plots of these pairs of quantities are shown in Figures 37 and 38.

The correlations of peak $\mathrm{H}_{2}$ surface density with the far-infrared luminosity and dust temperature suggest that galaxies with higher gas surface densities are more rapidly producing hot young stars, which in turn heat the gas and dust more efficiently. This correlation is consistent with the observed systematic correlation of the
HCN luminosity (which traces dense gas mass) with $L_{\mathrm{FIR}}(\mathrm{Gao} \&$ Solomon 2004). The far-infrared luminosity can be used to estimate the star formation rate (Kennicutt 1998), although there can also be a significant contribution from a buried AGN (Tran et al. 2001). Thus, this correlation suggests that higher star formation rates and/or AGN activity are associated with higher gas surface densities inside the central kiloparsec. (Note that this conclusion is somewhat different from the correlation between star formation efficiencies and gas surface densities seen by Scoville et al. [1991] and discussed in $\S 4.2$.) Interestingly, the total $\mathrm{H}_{2}$ mass detected with the SMA does not correlate with the far-infrared luminosity in our sample, although this may be partly due to the limited range of $L_{\mathrm{FIR}}$ in our sample (see Iono et al. [2008] for a discussion of this correlation in the context of a larger sample including high-redshift galaxies.) This lack of correlation suggests that it is the concentration of gas into the central regions that is important for generating the high star formation rates seen in these merging and merger-remnant galaxies, rather than the total amount of fuel available on somewhat larger scales. This result suggests that the increased star formation rate and/or AGN activity inferred for the more LIRGs is primarily a result of the 

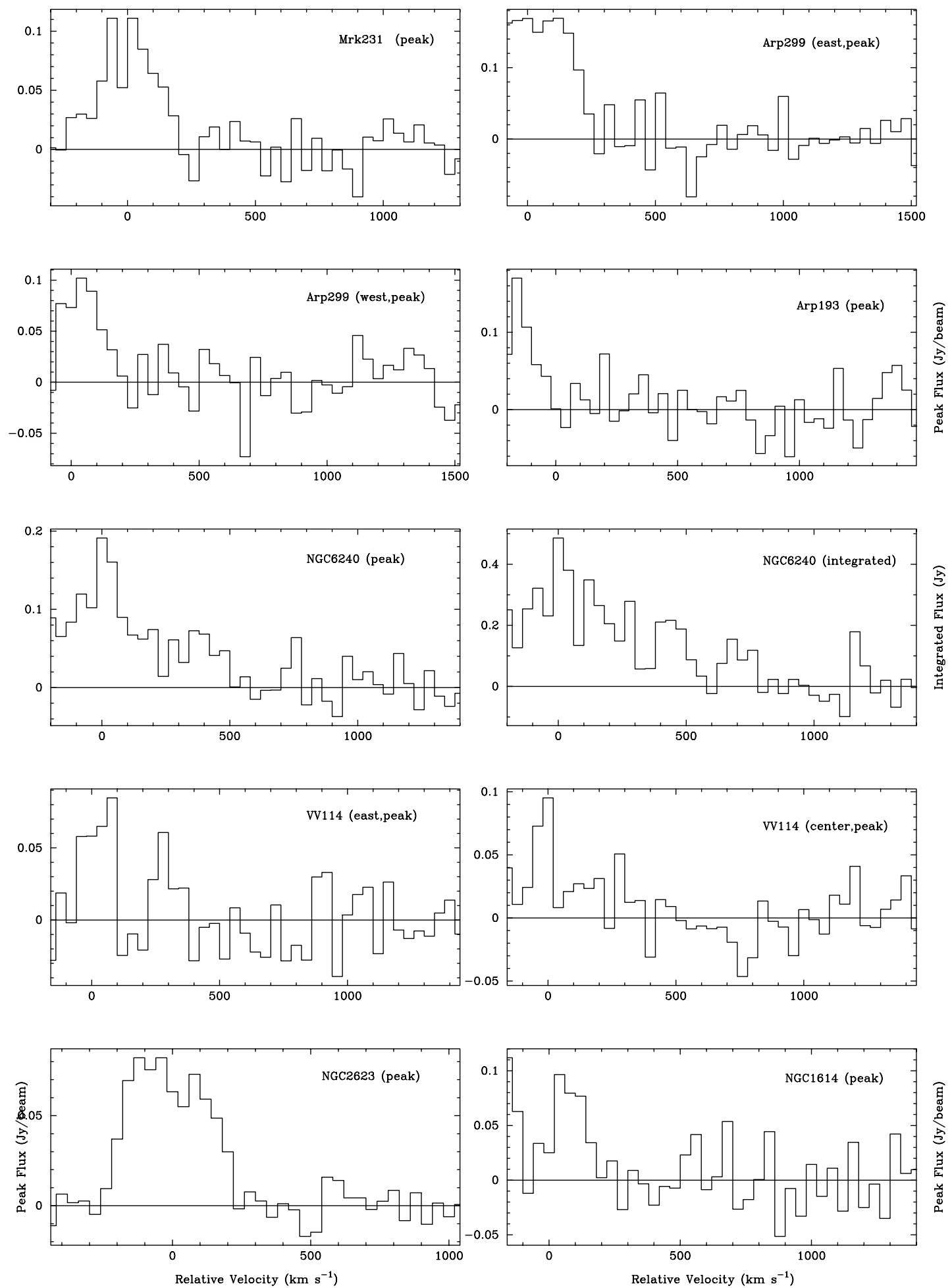

Fig. 36. - Peak spectra of the $\mathrm{HCO}^{+} J=4-3$ emission for nine galaxy components from our sample for which $\mathrm{HCO}^{+} J=4-3$ emission was detected. The integrated spectrum is also given for NGC 6240. See Fig. 25 for additional details.

increased availability of fuel for star formation in the central kiloparsec.

The $\mathrm{CO} J=3-2 / 2-1$ line ratio has been used as an indicator of temperature in the molecular gas (Wilson et al. 1997). However, for the galaxies in our sample, there is no obvious correlation between this ratio and the dust temperature derived from the 60 and $100 \mu \mathrm{m}$ IRAS data. One possible reason for this lack of correlation is that the derived dust temperatures are based on global measurements while the $\mathrm{CO} J=3-2 / 2-1$ line ratio traces material more concentrated to the center. The line ratio does correlate with both the total molecular gas mass and the beam area (Fig. 37). We can think of no obvious physical reason why the line ratio should correlate with the beam area, and so this may be produced by better sensitivity (particularly important for the CO 3-2 line) in the lower resolution data. A similar effect could produce the correlation with total mass, although it could also be hinting at a temperature dependence in the CO-to- $\mathrm{H}_{2}$ conversion factor, which would affect the derived mass. We do not 
TABLE 4

CO 3-2 Single Dish and Interferometric Fluxes

\begin{tabular}{|c|c|c|c|c|}
\hline Galaxy & $\begin{array}{c}\text { CO 3-2 (SMA) } \\
\left(\mathrm{Jy} \mathrm{km} \mathrm{s}^{-1}\right)\end{array}$ & 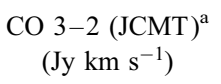 & $\begin{array}{c}\text { Missing Flux }{ }^{\mathrm{b}} \\
\left(\mathrm{Jy} \mathrm{km} \mathrm{s}^{-1}\right)\end{array}$ & $\begin{array}{l}\text { Missing Flux } \\
\text { (percent) }\end{array}$ \\
\hline IRAS $17208-0014 \ldots \ldots \ldots \ldots \ldots$ & $478 \pm 33$ & $985 \pm 128$ & $510 \pm 260$ & $51 \pm 15$ \\
\hline Mrk 231 & $308 \pm 8$ & $480 \pm 100$ & $170 \pm 150$ & $35 \pm 23$ \\
\hline Mrk $273 \ldots \ldots \ldots \ldots \ldots \ldots \ldots \ldots \ldots \ldots \ldots$ & $441 \pm 14$ & $410 \pm 90$ & 0 & 0 \\
\hline IRAS $10565+2448 \ldots \ldots \ldots \ldots \ldots \ldots$ & $204 \pm 7$ & $470 \pm 60$ & $270 \pm 120$ & $57 \pm 14$ \\
\hline 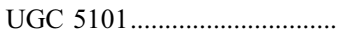 & $209 \pm 10$ & $490 \pm 80$ & $280 \pm 130$ & $57 \pm 14$ \\
\hline 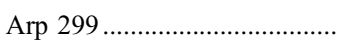 & $2582 \pm 57$ & $4890 \pm 180^{\mathrm{e}}$ & $2300 \pm 1600$ & $47 \pm 19$ \\
\hline Arp 55 & $146 \pm 9$ & $\geq 425 \pm 40^{\mathrm{c}}$ & $\geq 280 \pm 100$ & $\geq 66 \pm 10$ \\
\hline Arp 193. & $890 \pm 15$ & $1118 \pm 94$ & $230 \pm 300$ & $20 \pm 24$ \\
\hline 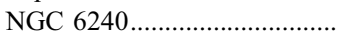 & $2428 \pm 52$ & $3205 \pm 642$ & $780 \pm 1030$ & $24 \pm 26$ \\
\hline 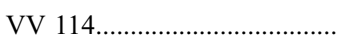 & $1530 \pm 16$ & $2956 \pm 133^{d}$ & $1430 \pm 680$ & $48 \pm 15$ \\
\hline NGC 5331S................................ & $401 \pm 24$ & $\ldots$ & $\ldots$ & $\ldots$ \\
\hline NGC 5331N........................... & $77 \pm 10$ & $\ldots$ & $\ldots$ & $\ldots$ \\
\hline 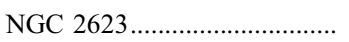 & $607 \pm 5$ & $620 \pm 44^{\mathrm{d}}$ & $13 \pm 190$ & $2 \pm 29$ \\
\hline 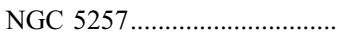 & $286 \pm 47$ & $437 \pm 67$ & $151 \pm 133$ & $36 \pm 24$ \\
\hline 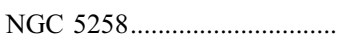 & $854 \pm 52$ & $504 \pm 26^{\mathrm{e}}$ & 0 & 0 \\
\hline 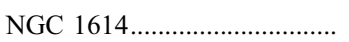 & $674 \pm 14$ & $1471 \pm 62^{\mathrm{d}}$ & $800 \pm 330$ & $54 \pm 13$ \\
\hline
\end{tabular}

${ }^{\mathrm{a}}$ For galaxies that were not mapped, conversions from $\mathrm{K}\left(T_{A}^{*}\right)$ to Jy were done assuming the source is point-like compared to the single dish beam, except for Arp 55 for which a main beam efficiency $\eta_{\mathrm{MB}}=0.63$ was used. The flux for NGC 6240 is taken from Greve et al. (2007).

b Uncertainty in missing flux assumes $20 \%$ calibration uncertainty on each flux except for NGC 5258 and Arp 299, for which a $30 \%$ calibration uncertainty in the JCMT data is assumed.

c Flux is from a single spectrum centered near the southwestern source.

d Flux is calculated from a $7 \times 7$ map with $5^{\prime \prime}$ spacing.

e Flux is calculated from a map made with HARP-B with $6{ }^{\prime \prime}$ spacing.

detect ${ }^{13} \mathrm{CO} J=2-1$ emission from enough of our galaxies to make similar correlation plots involving this line. We will present an analysis of the various $\mathrm{CO}$ line ratios, both integrated and spatially resolved, in combination with radiative transfer models in a future paper.

\subsection{Does the Central Gas Surface Density Correlate with $L_{\mathrm{FIR}} / M_{\mathrm{H}_{2}}$ ?}

Scoville et al. (1991) found that the central gas surface density increased as the ratio of far-infrared luminosity to nuclear gas mass increased. The far-infrared luminosity is a good tracer of the star formation rate (Kennicutt 1998); thus, if most of the farinfrared luminosity occurs in the central regions, then this ratio traces the star formation efficiency of the central, high-density starburst. Scoville et al. (1991) interpreted the observed trend as indicating that higher star formation efficiencies are produced by higher gas surface densities. However, our data do not show any evidence of a similar correlation (Fig. 39), and so it is worth examining further the possible explanations for the disagreement between these two results.

Scoville et al. (1991) adopted a standard CO-to- $\mathrm{H}_{2}$ conversion factor, and so their gas surface densities can be expected to be a

TABLE 5

Interferometric and Single Dish $880 \mu \mathrm{m}$ Continuum Fluxes

\begin{tabular}{|c|c|c|c|c|c|}
\hline Galaxy & $\begin{array}{l}\text { JCMT Flux } \\
\text { (mJy) }\end{array}$ & $\begin{array}{c}\mathrm{CO} 3-2^{\mathrm{b}} \\
(\mathrm{mJy})\end{array}$ & $\begin{array}{l}\text { SMA Flux } \\
(\mathrm{mJy})\end{array}$ & $\begin{array}{l}\text { Missing Flux }{ }^{\mathrm{c}} \\
\quad(\mathrm{mJy})\end{array}$ & Percent Missing Flux ${ }^{\mathrm{c}}$ \\
\hline 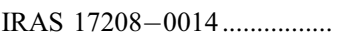 & $137 \pm 41$ & 18.9 & $48 \pm 10$ & $70 \pm 31$ & $59 \pm 14$ \\
\hline Mrk 231 & $69 \pm 13$ & 9.2 & $80 \pm 4$ & 0 & 0 \\
\hline 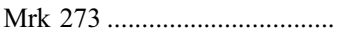 & $60 \pm 16$ & 8.4 & $56 \pm 5$ & 0 & 0 \\
\hline IRAS $10565+2448 \ldots \ldots \ldots \ldots \ldots \ldots$ & $54 \pm 12$ & 9.0 & $15 \pm 3$ & $30 \pm 15$ & $67 \pm 13$ \\
\hline UGC $5101 \ldots \ldots \ldots \ldots \ldots \ldots \ldots \ldots \ldots$ & $102 \pm 18$ & 9.4 & $37 \pm 9$ & $56 \pm 30$ & $60 \pm 17$ \\
\hline Arp 299 & $376 \pm 10$ & 93.7 & $101 \pm 7$ & $181 \pm 69$ & $64 \pm 10$ \\
\hline Arp 55 & $58 \pm 12$ & $\geq 8.1$ & $26 \pm 7$ & $\leq 23 \pm 18$ & $\leq 47 \pm 22$ \\
\hline Arp 193 & $100 \pm 13$ & 21.4 & $39 \pm 4$ & $40 \pm 24$ & $50 \pm 16$ \\
\hline NGC $6240 \ldots \ldots \ldots$ & $133 \pm 40$ & 61.4 & $33 \pm 13$ & $38 \pm 30$ & $54 \pm 22$ \\
\hline 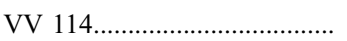 & $181 \pm 8$ & 56.7 & $26 \pm 6$ & $99 \pm 33$ & $79 \pm 07$ \\
\hline 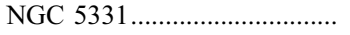 & $64 \pm 6$ & 9.2 & $27 \pm 6$ & $28 \pm 15$ & $50 \pm 18$ \\
\hline NGC 2623 & $81 \pm 12$ & 11.9 & $50 \pm 2$ & $19 \pm 21$ & $27 \pm 23$ \\
\hline 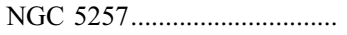 & $101 \pm 20$ & 8.4 & $<26$ & $>66 \pm 28$ & $>72 \pm 17$ \\
\hline NGC $5258 \ldots \ldots \ldots$ & $150 \pm 28$ & 16.4 & $104 \pm 21$ & $29 \pm 46$ & $22 \pm 29$ \\
\hline NGC 1614 & $194 \pm 29$ & 28.2 & $27 \pm 7$ & $139 \pm 45$ & $84 \pm 6$ \\
\hline
\end{tabular}

${ }^{\text {a }}$ See text for a description of the source of the uncertainty listed here for each galaxy.

b SMA CO $J=3-2$ fluxes used for Mrk 273, NGC 5258, and NGC 5331. Arp 55 flux is likely underestimated since spectrum was centered near the southwestern source and so misses most emission from the stronger northeastern source.

${ }^{c}$ Uncertainty in missing flux assumes $20 \%$ calibration uncertainty on each flux. 
TABLE 6

Gas and Dust Masses from Total Luminosity

\begin{tabular}{|c|c|c|c|c|c|c|}
\hline Galaxy & $\begin{array}{c}D_{L} \\
(\mathrm{Mpc})\end{array}$ & $\begin{array}{c}L_{\mathrm{CO}}^{\prime}(3-2) \\
\left(10^{9} \mathrm{Km} \mathrm{s}^{-1} \mathrm{pc}^{2}\right)\end{array}$ & $\begin{array}{l}T_{D}^{\mathrm{a}} \\
(\mathrm{K})\end{array}$ & $\begin{array}{c}M_{\text {dust }} \\
\left(10^{7} M_{\odot}\right)\end{array}$ & $\begin{array}{c}M_{\mathrm{H}_{2}}{ }^{\mathrm{b}} \\
\left(10^{9} M_{\odot}\right)\end{array}$ & Gas/Dust \\
\hline IRAS $17208-0014 \ldots$. & 189 & 4.46 & 41.3 & $4.32 \pm 0.90^{\mathrm{c}}$ & $7.13 \pm 0.49$ & $165 \pm 36^{\mathrm{c}}$ \\
\hline Mrk 231 & 179 & 2.58 & 43.1 & $7.67 \pm 0.38^{\mathrm{d}}$ & $4.12 \pm 0.11$ & $54 \pm 3^{\mathrm{d}}$ \\
\hline Mrk 273 .......................................... & 166 & 3.19 & 43.0 & $6.17 \pm 0.55^{\mathrm{e}}$ & $5.10 \pm 0.16$ & $83 \pm 8^{\mathrm{e}}$ \\
\hline IRAS $10565+2448 \ldots \ldots \ldots \ldots \ldots \ldots \ldots$ & 191 & 1.94 & 38.6 & $2.50 \pm 0.50$ & $3.11 \pm 0.11$ & $124 \pm 25$ \\
\hline UGC $5101 \ldots \ldots \ldots \ldots \ldots \ldots \ldots \ldots$ & 174 & 1.66 & 35.5 & $5.67 \pm 1.38^{\mathrm{e}}$ & $2.65 \pm 0.13$ & $47 \pm 11^{\mathrm{e}}$ \\
\hline IC 694 (Arp 299) ............................ & 44 & 0.840 & 41.5 & $0.655 \pm 0.040^{\mathrm{e}}$ & $1.34 \pm 0.02$ & $205 \pm 13^{\mathrm{e}}$ \\
\hline NGC 3690 (Arp 299) ...................... & 44 & 0.311 & 41.5 & $0.162 \pm 0.040$ & $0.497 \pm 0.014$ & $308 \pm 77$ \\
\hline Arp 55(NE) .................................... & 173 & 0.737 & 34.5 & $4.09 \pm 1.10$ & $1.18 \pm 0.11$ & $29 \pm 8$ \\
\hline Arp 55(SW) .................................... & 173 & 0.408 & 34.5 & $<1.26 \pm 0.63$ & $0.652 \pm 0.100$ & $>52 \pm 27$ \\
\hline Arp 193=IC 883 & 102 & 2.45 & 35.4 & $2.06 \pm 0.21$ & $3.92 \pm 0.07$ & $190 \pm 20$ \\
\hline 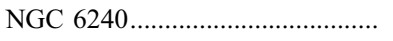 & 107 & 7.39 & 40.4 & $1.63 \pm 0.64$ & $11.82 \pm 0.25$ & $725 \pm 286$ \\
\hline 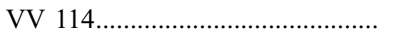 & 87 & 3.09 & 39.4 & $0.876 \pm 0.202$ & $4.94 \pm 0.05$ & $565 \pm 130$ \\
\hline 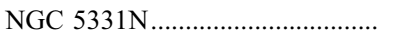 & 145 & 0.427 & 32.1 & $<1.45 \pm 0.73$ & $0.682 \pm 0.089$ & $>47 \pm 24$ \\
\hline NGC 5331S..................................... & 145 & 2.22 & 32.1 & $3.27 \pm 0.73$ & $3.55 \pm 0.21$ & $109 \pm 25$ \\
\hline NGC 2623 .................................... & 80 & 1.04 & 39.9 & $1.40 \pm 0.06^{\mathrm{e}}$ & $1.66 \pm 0.01$ & $118 \pm 4^{\mathrm{e}}$ \\
\hline NGC 5257......................................... & 99 & 0.746 & 36.0 & $<1.27 \pm 0.63$ & $1.19 \pm 0.20$ & $>94 \pm 50$ \\
\hline 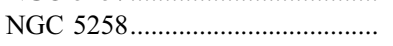 & 99 & 2.23 & 36.0 & $5.07 \pm 1.02^{\mathrm{f}}$ & $3.56 \pm 0.22$ & $70 \pm 15^{\mathrm{f}}$ \\
\hline 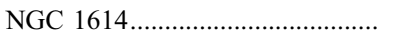 & 69 & 0.860 & 42.5 & $0.313 \pm 0.081^{\mathrm{c}}$ & $1.38 \pm 0.03$ & $440 \pm 114^{\mathrm{c}}$ \\
\hline
\end{tabular}

${ }^{\text {a }} T_{e}$ is the dust temperature calculated from fitting a modified blackbody function as in Klaas et al. (2001) to published data from 60 to $800 \mu \mathrm{m}$; see text.

${ }^{b} M_{\mathrm{H}_{2}}$ calculated assuming CO $3-2 / \mathrm{CO} 1-0=0.5$ and $M_{\mathrm{H}_{2}}=0.8 L_{\mathrm{CO}}^{\prime}(1-0)$ (Downes \& Solomon 1998).

${ }^{\mathrm{c}}$ Dust mass and gas-to-dust ratio have been corrected by a factor of 0.6 to account for a nonthermal contribution to the $880 \mu \mathrm{m}$ flux. See text.

${ }^{\mathrm{d}}$ Dust mass and gas-to-dust ratio have been corrected by a factor of 0.75 to account for a nonthermal contribution to the $880 \mu \mathrm{m}$ flux. See text.

e There may be a nonthermal contribution to the continuum flux of this source; however, any effect on the dust mass and the gas-to-dust ratio is likely smaller than the uncertainties. See text.

${ }^{\mathrm{f}}$ NGC 5258 contains an off-nuclear continuum source, whose flux is used here, while the CO $J=3-2$ emission comes primarily from the extended southern arm. Thus, the dust mass and gas-to-dust ratio given here are likely incorrect.

TABLE 7

Gas and Dust Masses in a Single Beam

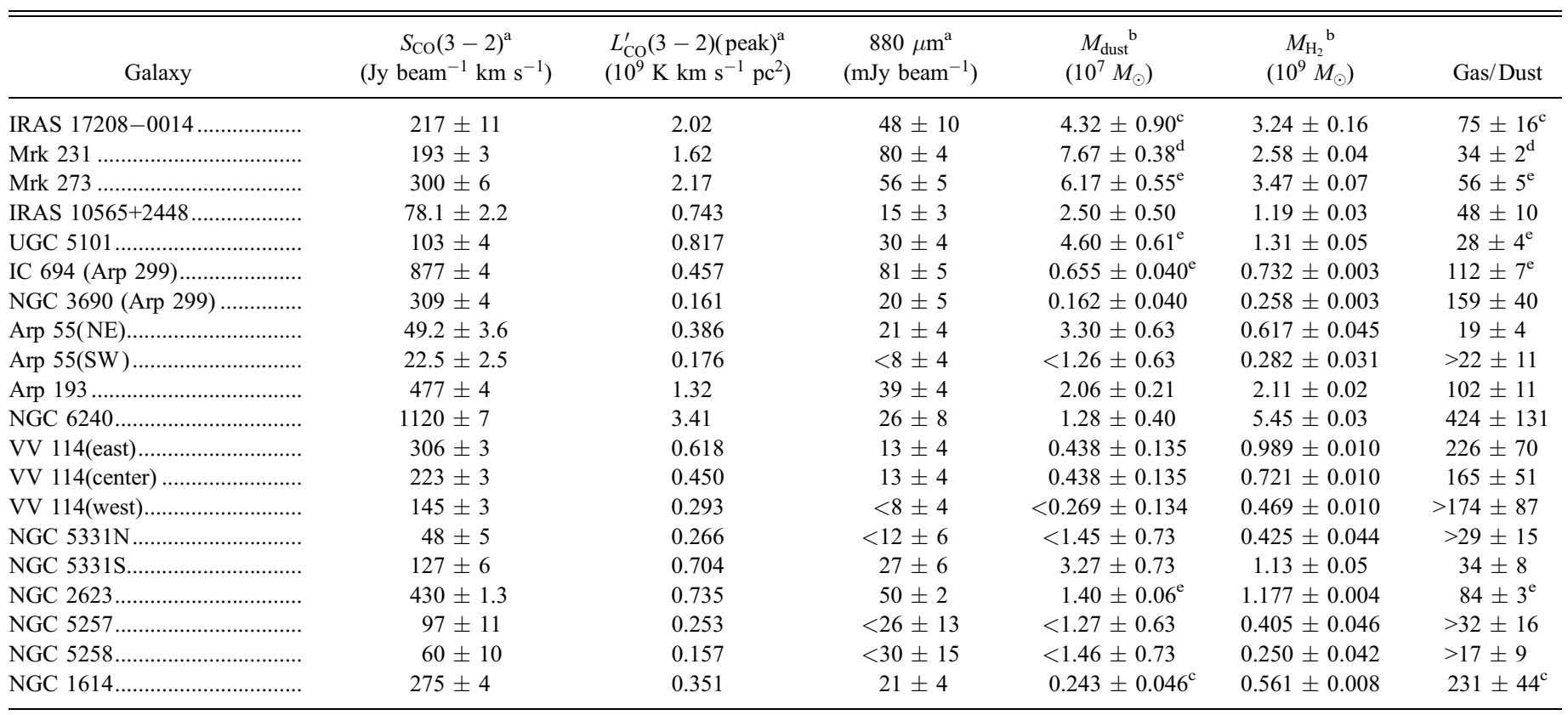

a Values measured at the peak emission except for NGC 5258, where it is measured at the peak in the nuclear region. See Table 2 for the beam size for each galaxy.

${ }^{\mathrm{b}} M_{\mathrm{H}_{2}}$ and $M_{\text {dust }}$ are calculated with the same assumptions and dust temperature used in Table 6 .

${ }^{c}$ Dust mass and gas-to-dust ratio have been corrected by a factor of 0.6 to account for a possible nonthermal contribution to the $880 \mu \mathrm{m}$ flux. See text.

${ }^{\mathrm{d}}$ Dust mass and gas-to-dust ratio have been corrected by a factor of 0.75 to account for a nonthermal contribution to the $880 \mu \mathrm{m}$ flux. See text.

e There may be a nonthermal contribution to the continuum flux of this source; however, any effect on the dust mass and the gas-to-dust ratio is likely smaller than the uncertainties. See text. 
TABLE 8

Probability $p$ of a False Correlation after Removing the Effect of Distance

\begin{tabular}{|c|c|c|c|c|c|c|c|c|}
\hline Quantity & $T_{D}$ & $\Sigma_{\mathrm{H}_{2}}($ peak $)$ & Beam Area & $M_{\mathrm{H}_{2}}($ total $)$ & $L_{\mathrm{FIR}} / M_{\mathrm{H}_{2}}($ total $)$ & $L_{\mathrm{FIR}} / M_{\mathrm{H}_{2}}($ peak $)$ & $\begin{array}{l}\text { Gas-to-Dust } \\
\text { Ratio (peak) }\end{array}$ & $\begin{array}{c}\mathrm{CO} J=3-2 / 2-1 \\
\text { Line Ratio }\end{array}$ \\
\hline$L_{\mathrm{FIR}}$ & 0.0168 & 0.0014 & 0.0552 & 0.2244 & 0.0250 & 0.2042 & 0.5774 & 0.4554 \\
\hline$\Sigma_{\mathrm{H}_{2}}($ peak $)$ & $\ldots$ & $\ldots$ & 0.0063 & 0.2084 & 0.1219 & 0.9933 & 0.7035 & 0.7662 \\
\hline 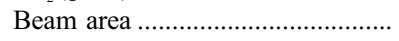 & $\ldots$ & $\ldots$ & $\ldots$ & 0.1202 & 0.0284 & 0.5258 & 0.5393 & 0.0005 \\
\hline$M_{\mathrm{H}_{2}}($ total $)$ & $\ldots$ & $\ldots$ & $\ldots$ & $\ldots$ & 0.2107 & 0.6571 & 0.1044 & 0.0074 \\
\hline Gas-to-dust ratio (peak).................. & $\ldots$ & $\ldots$ & $\ldots$ & $\ldots$ & $\ldots$ & $\ldots$ & $\ldots$ & 0.0578 \\
\hline
\end{tabular}

factor of 6 higher and their infrared luminosity to $\mathrm{H}_{2}$ mass ratios a factor of 6 lower than our values. However, this would only shift the absolute scale of their correlation and not change the correlation itself. Indeed, when we estimate their relationship and correct for the different gas masses, we find that it agrees well with the location of most of the points in our analysis (Fig. 39). Scoville et al. (1991) used the CO $J=1-0$ transition in their study, which is more sensitive to cooler, more spatially extended emission than is the $\mathrm{CO} J=3-2$ transition used here. However, by
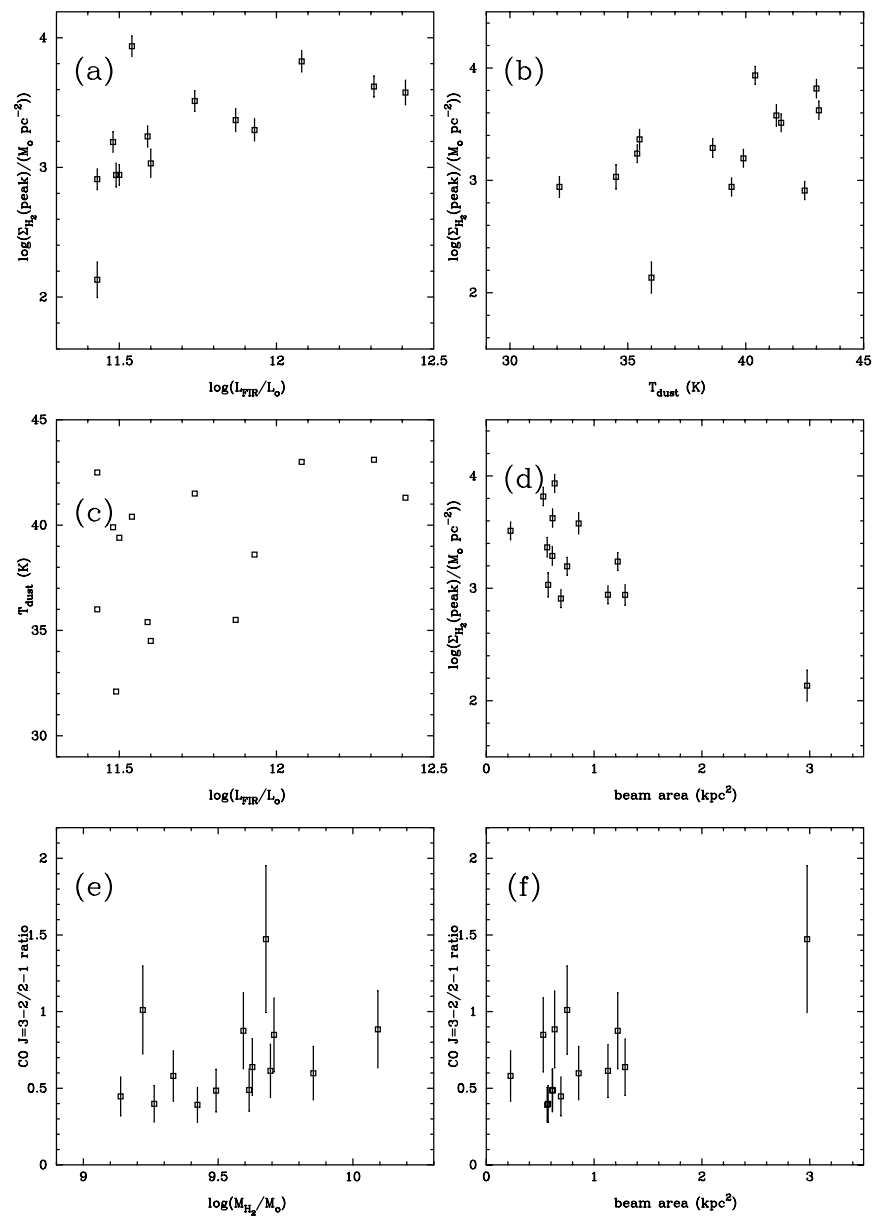

FIG. 37.-Pairs of quantities for the galaxies in our sample that showed significant correlations. (a) Peak $\mathrm{H}_{2}$ surface density vs. far-infrared luminosity. (b) Peak $\mathrm{H}_{2}$ surface density vs. dust temperature. (c) Dust temperature vs. farinfrared luminosity. (d) Peak $\mathrm{H}_{2}$ surface density vs. beam area. Note that this correlation depends heavily on the two galaxies with the most divergent beam areas. (e) $\mathrm{CO} J=3-2 / 2-1$ line ratio vs. total $\mathrm{H}_{2}$ mass detected with the SMA. ( $f$ ) $\mathrm{CO}$ $J=3-2 / 2-1$ line ratio vs. beam area. isolating the nuclear emission from any more extended emission, Scoville et al. (1991) may have mitigated the contribution of any extended disk gas in their study.

An additional issue worth considering is the possibility of different sample selection or observational biases. Our galaxy sample spans a slightly smaller range of central gas surface density (a factor of 60) than does the sample in Scoville et al. (1991), which had a range of a factor of 100 . However, our range of surface density drops to only a factor of 10 if the galaxy with the poorest spatial resolution (NGC 5257) is removed from the sample. On the other hand, some of the large range in Scoville et al.
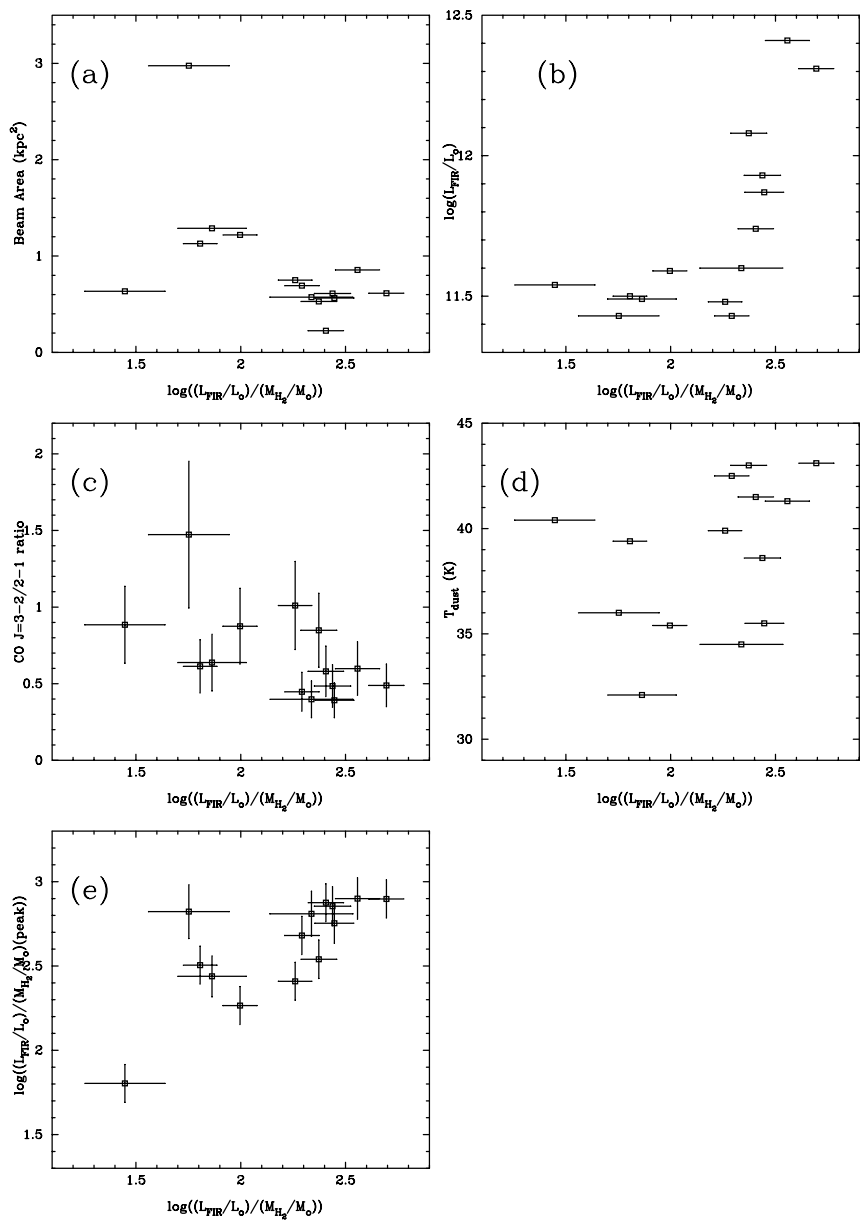

FIG. 38. - Correlations of five quantities with the ratio $L_{\mathrm{FIR}} / M_{\mathrm{H}_{2}}$, where $M_{\mathrm{H}_{2}}$ is the total mass detected with the SMA. All these correlations become insignificant if one of NGC 5257 or Arp 299 is removed from the analysis. (a) Beam area. (b) Farinfrared luminosity $L_{\mathrm{FIR}}$. (c) $\mathrm{CO} J=3-2 / 2-1$ line ratio. (d) Dust temperature, (e) Ratio of far-infrared luminosity to peak $\mathrm{H}_{2}$ mass. 


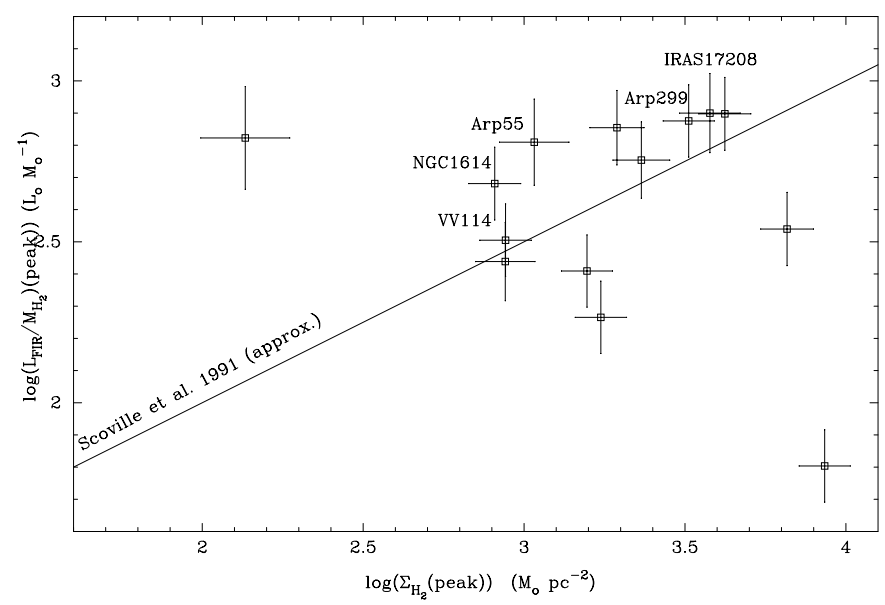

FIG. 39.- Ratio of global far-infrared luminosity to the peak $\mathrm{H}_{2}$ gas mass detected with the SMA vs. peak $\mathrm{H}_{2}$ surface density. Only the highest surface density component is shown for composite systems. The error bars shown represent measurement errors on the $\mathrm{CO} J=3-2$ emission as well as a $20 \%$ calibration uncertainty. Note that the calibration uncertainty has the same systematic effect on both quantities, since both are derived from the same data set. No correlation is seen, in conflict with the earlier study by Scoville et al. (1991). The approximate relationship seen by Scoville et al. (1991; corrected by a factor of 6 to account for the different $\mathrm{CO}-\mathrm{to}_{\mathrm{H}} \mathrm{H}_{2}$ conversion factor) is shown as the straight line. The five galaxies in common between this study and that of Scoville et al. (1991) are labeled on the plot.

(1991) may be due to the range of spatial resolution discussed below. The sample in Scoville et al. (1991) has a factor of 10 larger range in $L_{\mathrm{FIR}}$ and a factor of 5 larger range in the nuclear or peak gas mass. However, the net result is that the range of $L_{\mathrm{FIR}} / M_{\mathrm{H}_{2}}$ (a factor of 10) is similar in the two studies (although the range in our sample drops to a factor of only 4 if we remove NGC 6240). Scoville et al. (1991) calculated this ratio using only the gas mass in the central nuclear source that was also used to calculate the gas surface density, while we have used the peak $\mathrm{H}_{2}$ mass in the central beam, which would be a difference in the method if many of the sources in the Scoville et al. (1991) sample were spatially resolved. However, most of the sources in the Scoville et al. (1991) sample appear to have been unresolved in the original data sets, which implies that the method used to calculate the surface density and the $L_{\mathrm{FIR}} / M_{\mathrm{H}}$ ratio is very similar in this paper and in Scoville et al. (1991).

Scoville et al. (1991) used CO $J=1-0$ data for a sample of 14 galaxies, including five galaxies from our sample, with resolutions ranging from 0.1 to $3.2 \mathrm{kpc}$. Although their range of resolution is similar to that of the sample presented here, the distribution of resolutions is much broader than for our data set, where all but two galaxies (Arp 299 and NGC 5257/8) have resolutions between 0.7 and $1.1 \mathrm{kpc}$. This distribution of resolutions might introduce systematic effects into the analysis of Scoville et al. (1991) in the sense that galaxies observed with better angular resolutions could have higher gas surface densities. Indeed, the average resolution for the seven galaxies at the high end of the correlation is $0.66 \mathrm{kpc}$, while the average resolution for the remaining seven galaxies is $1.14 \mathrm{kpc}$.

To examine the effect of resolution further, we looked at the five galaxies that are common to both samples (Arp 299/IC 694, IRAS 17208-0014, Arp 55, VV 114, and NGC 1614). The values of surface density and $L_{\mathrm{FIR}} / M_{\mathrm{H}_{2}}$ ratio are very similar for Arp 299 and NGC 1614, which are two galaxies for which the angular resolutions of the $\mathrm{CO} J=1-0$ and the $\mathrm{CO} J=3-2$ data agree within a factor of 2. Both IRAS 17208-0014 and VV 114 move to higher surface densities and $L_{\mathrm{FIR}} / M_{\mathrm{H}_{2}}$ ratios using the CO $J=3-2$ data, which have angular resolutions a factor of
$2-3$ better than the $\mathrm{CO} J=1-0$ data. The most dramatic change is for Arp 55, for which the $\operatorname{CO} J=3-2$ data have a factor of 8 better angular resolution; its surface density and $L_{\mathrm{FIR}} / M_{\mathrm{H}_{2}}$ ratio increase dramatically, to the point where it is larger in both quantities than NGC 1614.

In summary, the better uniformity in angular resolution in our sample compared to that of Scoville et al. (1991) gives us confidence that the lack of correlation seen between the peak $\mathrm{H}_{2}$ surface density and the ratio of infrared luminosity to $\mathrm{H}_{2}$ mass seen in our analysis is a real effect and not an artifact of our sample selection or observing techniques. While such a correlation may exist for galaxies with lower far-infrared luminosities (below the luminosity cutoff of our sample), it cannot help us in understanding the high star formation rates in the luminous systems studied here. The data presented here imply a star formation rate $(\S 4)$, not a star formation efficiency, that increases with the central gas surface density. We will explore these interesting correlations further in a future paper.

\section{CONCLUSIONS}

In this paper, we have presented new data obtained with the Submillimeter Array for a sample of 14 luminous and ultraluminous infrared galaxies selected to have distances $D_{L}<200 \mathrm{Mpc}$ and far-infrared luminosities $\log L_{\mathrm{FIR}}>11.4$. We have obtained data in the $\mathrm{CO} J=3-2, \mathrm{CO} J=2-1,{ }^{13} \mathrm{CO} J=2-1$, and $\mathrm{HCO}^{+}$ $J=4-3$ lines as well as continuum data at $880 \mu \mathrm{m}$ and $1.3 \mathrm{~mm}$ with spatial resolutions of order $1 \mathrm{kpc}$ or better in all but one of the target galaxies. We present integrated intensity, velocity field, and velocity dispersion maps for the ${ }^{12} \mathrm{CO}$ lines, integrated intensity maps for the continuum, ${ }^{13} \mathrm{CO}$, and $\mathrm{HCO}^{+}$lines, and peak and integrated spectra for all the detected lines.

We have compared our $\operatorname{CO} J=3-2$ and $880 \mu \mathrm{m}$ continuum fluxes detected with the SMA with published, archival, and new data from the James Clerk Maxwell Telescope. This comparison shows that the interferometric data miss a significant fraction (typically $50 \%$ ) of the $\mathrm{CO} J=3-2$ emission for eight of the galaxies in our sample and also a significant fraction (typically $50 \%-80 \%$ ) of the continuum flux for nine of the galaxies. This large amount of missing continuum flux suggests that a significant fraction of the $880 \mu \mathrm{m}$ emission in these systems occurs on moderately large spatial scales. The good agreement between the percentage of missing flux seen in the $\mathrm{CO} J=3-2$ line and the $880 \mu \mathrm{m}$ continuum suggests that the missing continuum flux comes from dust emission associated with molecular gas in the more extended disks of the galaxies.

We have combined our $\mathrm{CO}$ and continuum data to determine the gas-to-dust mass ratio in the central regions of these galaxies. We adopt the smaller value of the $\mathrm{CO}-\mathrm{to}-\mathrm{H}_{2}$ conversion factor from Downes \& Solomon (1998) and calculate the dust temperature by fitting a modified blackbody function as in Klaas et al. (2001). Because of the lower signal-to-noise ratio in the continuum data, we find that we obtain more consistent measurements of the gas-to-dust mass ratio if we use a single beam to probe the central region of each galaxy or galaxy component. We find an average gas-to-dust mass ratio of $120 \pm 28$ (rms deviation 109), very similar to the value of 150 determined for the Milky Way. This similarity between the gas-to-dust ratio in these luminous systems and that in the Milky Way is somewhat surprising, given that the dust is subject to more intense heating from the starburst and possibly accretion activity compared to typical regions in the Milky Way.

We have searched for correlations among nine physical and observational quantities for the galaxies in our sample. We find five correlations that appear to be statistically significant as well 
as robust to small changes in the exact galaxy sample. The most interesting correlation is that of peak $\mathrm{H}_{2}$ surface density with the far-infrared luminosity. Since the far-infrared luminosity can be used to estimate the star formation rate, these correlations suggest that galaxies with higher gas surface densities inside the central kiloparsec have a higher star formation rate. We do not see a significant correlation of total $\mathrm{H}_{2}$ mass with the far-infrared luminosity, which suggests that the increase in star formation rate is due to the increased availability of molecular gas as fuel for star formation in the central regions, rather than the total amount of gas available on somewhat larger scales.

Our data do not show any evidence of a significant correlation between central gas surface density and the ratio of far-infrared luminosity to nuclear gas mass. This lack of correlation is different from the results of Scoville et al. (1991), who interpreted their observed correlation as indicating that higher star formation efficiencies result from higher gas surface densities. We suggest that the correlation seen by Scoville et al. (1991) was produced by the wider distribution of spatial resolutions in their data set and is not an intrinsic property of these very luminous galaxies. To reiterate, our new data show that it is star formation rate, not star formation efficiency, that increases with the central gas surface density in LIRGs and ULIRGs

There are a number of additional papers in preparation or planning that will present more detailed analysis of various aspects of the data. We will compare the results from this survey with similar observations of high-redshift submillimeter galaxies to study the gas properties of a wide range of luminous galaxies using the $\mathrm{CO} J=3-2$ line to trace the molecular gas content (Iono et al. 2008). A detailed analysis of the molecular gas properties of NGC 6240 has already been presented in Iono et al. (2007); we will present similar detailed analyses of the gas, dust, and star formation properties individual galaxies such as VV 114 (G. R. Petitpas et al. 2008, in preparation) and Arp 299. We will examine the physical properties of the molecular gas for the entire sample using spatially resolved radiative transfer models, similar to what has been done for NGC 6240 (Iono et al. 2007), as well as carry out dynamical analysis and modeling of the galaxies, both of which can give an independent estimate of the CO-to- $\mathrm{H}_{2}$ conversion factor, similar to the analysis of Downes \& Solomon (1998). This physical analysis will also allow us to place constraints on the origin of the $\mathrm{OH}$ megamaser activity in LIRGs (Darling 2007). We will combine our high-resolution SMA data with Spitzer data to compare the properties of the warm and cold dust (see also Armus et al. 2007; Marshall et al. 2007). Finally, we will make detailed comparisons between the molecular gas and dust properties of these U/LIRGs and the predictions of numerical simulations of merging galaxies (e.g., Cox et al. 2006; Chakrabarti et al. 2007).

The Submillimeter Array is a joint project between the Smithsonian Astrophysical Observatory and the Academia Sinica Institute of Astronomy and Astrophysics and is funded by the Smithsonian Institution and the Academia Sinica. The James Clerk Maxwell Telescope is operated by The Joint Astronomy Centre on behalf of the Particle Physics and Astronomy Research Council of the United Kingdom, the Netherlands Organisation for Scientific Research, and the National Research Council of Canada. This research has made use of the NASA/IPAC Extragalactic Database (NED), which is operated by the Jet Propulsion Laboratory, California Institute of Technology, under contract with the National Aeronautics and Space Administration. We are grateful to the many SMA observers who helped to take the data presented in this paper and to the SMA TAC for giving this project a high priority. We thank James di Francesco for making available to us the pipeline processed archival SCUBA data for VV 114, NGC 5331, and Arp 299, Padelis Papadopoulos for access to his single dish CO data, and the anonymous referee for a very useful and prompt referee report. C. D. W. and J. G. acknowledge support by the Natural Science and Engineering Research Council of Canada (NSERC). A. J. B. acknowledges support by National Science Foundation grant AST 07-08653. M. J. acknowledges support by the Academy of Finland grant 124620.

Facilities: SMA, JCMT.

\section{APPENDIX}

\section{DISCUSSION OF INDIVIDUAL GALAXIES}

\section{A1. IRAS $17208-0014$}

Veilleux et al. (1995) classify the optical spectrum of IRAS 17208-0014 as H II. High-resolution near-infrared imaging shows no direct evidence of an AGN, as the nuclear emission is extended in all bands (Scoville et al. 2000). The [Ne v] line, which is an indicator of an AGN, is not detected in this galaxy (Farrah et al. 2007). High-resolution radio imaging at $4.8 \mathrm{GHz}$ also shows no evidence for an AGN (Baan \& Klöckner 2006). Near-infrared images show extended emission with a brighter nucleus (Scoville et al. 2000).

However, the millimeter continuum fluxes in Table 3 suggest there may be a significant nonthermal or free-free component present. The $880 \mu \mathrm{m}$ and $1.4 \mathrm{~mm}$ continuum fluxes are inconsistent with dust with $\beta=1.5$ at greater than the $1.5 \sigma$ level even after including $20 \%$ calibration uncertainties. If we assume that any nonthermal component depends inversely on frequency (Condon 1992 ) while the dust component varies as $\nu^{3.5}$ (dust emissivity $\beta=1.5$ ), then the nonthermal emission would contribute $40 \%$ of the flux at $880 \mu \mathrm{m}$.

\section{A2. Mrk 231 (UGC 8058, VII Zw 490)}

Mrk 231 is the only object in our sample to show strong emission from an AGN. Veilleux et al. (1995) classify the optical spectrum as Seyfert 2, while Genzel et al. (1998) suggest an AGN-like radiation field based on mid-infrared emission lines and limits. Smith et al. (1998) suggest an AGN based on their $22 \mathrm{GHz}$ VLA data; Lonsdale et al. (2003) find that VLBI imaging data are most consistent with an AGN morphology, although they point out that the AGN may not be responsible for the majority of the bolometric luminosity of the galaxy. However, Davies et al. (2004) find that the nuclear starburst contributes $25 \%-40 \%$ of the bolometric luminosity. Mrk 231 also contains a hard X-ray source that is variable on timescales of a few hours (Gallagher et al. 2002).

The millimeter continuum fluxes in Table 3 suggest there is a significant nonthermal component present. Downes \& Solomon (1998) attributed all but $20 \%$ of their $1.3 \mathrm{~mm}$ continuum flux to nonthermal emission. If we assume that any nonthermal component 
depends inversely on frequency (Condon 1992) while the dust component varies as $\nu^{3.5}$ (dust emissivity $\beta=1.5$ ), then the nonthermal emission contributes perhaps $25 \%$ of the flux at $880 \mu \mathrm{m}$.

\section{A3. Mrk 273 (UGC 8696, VV 851, I Zw 71)}

Veilleux et al. (1995) classify the optical spectrum of Mrk 273 as LINER. Genzel et al. (1998) suggest that the mid-infrared radiation has equal contributions from a starburst and an AGN. Armus et al. (2007) detect both [Ne v] emission and continuum emission from dust hotter than $300 \mathrm{~K}$, both of which indicate the presence of an AGN. Xia et al. (2002) detect hard X-ray emission from the northern nucleus, while Ptak et al. (2003) detect the Fe line in X-rays, both suggesting the presence of an AGN. However, Smith et al. (1998) find that the compact $22 \mathrm{GHz}$ core can be fit by a model of clustered supernovae. Near-infrared images show extended emission with two bright central sources (Scoville et al. 2000).

The relative strength of the 1.3 and $2.6 \mathrm{~mm}$ continuum emission (Downes \& Solomon 1998) suggests that some nonthermal emission is present. However, the contribution at $880 \mu \mathrm{m}$ would be small and well within the $20 \%$ calibration uncertainty of the data.

\section{A4. IRAS $10565+2448$}

This galaxy has been relatively poorly studied. Veilleux et al. (1995) classify the optical spectrum of IRAS $10565+2448$ as H II. Condon et al. (1991) do not find any compact radio continuum peak at $1.49 \mathrm{GHz}$. Near-infrared images show extended emission with a brighter nucleus (Scoville et al. 2000). The [Ne v] line, which is an indicator of an AGN, is not detected in this galaxy (Farrah et al. 2007).

The millimeter continuum data are consistent with pure dust emission, although the $1.3 \mathrm{~mm}$ continuum point is on the large side of what is possible, leaving open the possibility of a small nonthermal contribution at $880 \mu \mathrm{m}$.

\section{A5. UGC 5101}

Veilleux et al. (1995) classify the optical spectrum of UGC 5101 as LINER. Although Genzel et al. (1998) suggest the mid-infrared radiation field is more similar to that of a starburst than an AGN, Armus et al. (2004) find that [Ne v] emission implies this source contains a buried AGN. Armus et al. (2007) detect continuum emission from dust hotter than $300 \mathrm{~K}$, which also indicates the presence of an AGN. Imanishi et al. (2003) detect hard X-ray emission, including the Fe line, which they interpret as evidence for a buried AGN. Lonsdale et al. (2003) use VLBI imaging to find that an AGN is responsible for at least $10 \%$ of the total radio flux. Near-infrared images show extended emission with a brighter nucleus (Scoville et al. 2000).

The millimeter continuum data are consistent with pure dust emission, although the $1.3 \mathrm{~mm}$ continuum point is on the large side of what is possible, leaving open the possibility of a small nonthermal contribution at $880 \mu \mathrm{m}$.

\section{A6. Arp 299 (VV 118, NGC 3690, Mrk 171, IC 694)}

High-resolution radio images from 1.4 to $8.4 \mathrm{GHz}$ reveal five bright compact sources as well as extended emission (Neff et al. 2004). The bright compact radio source in the nucleus of IC 694 breaks up into five candidate radio supernova remnants when observed with the VLBA; one of the sources may possibly be a low-luminosity AGN (Neff et al. 2004). X-ray images reveal a population of compact sources, including sources in the nucleus of each component which are likely to be AGN (Zezas et al. 2003). Further evidence for an AGN in NGC 3690 comes from García-Marín et al. (2006), who identify a conical region with Seyfert-like excitation emanating from the $\mathrm{B} 1$ region. Mid-infrared images show three compact sources associated with the two nuclei as well as the emission region north of NGC 3690 (Soifer et al. 2001); near-infrared images reveal a fourth compact source near NGC 3690 as well as extended emission (Alonso-Herrero et al. 2000)

The combination of the $2.6 \mathrm{~mm}$ continuum flux from Aalto et al. (1997) with the $1.3 \mathrm{~mm}$ continuum flux from this paper suggests that some nonthermal component may be present in the eastern component (IC 694). If we assume the nonthermal emission scales inversely with frequency and that all of the $2.6 \mathrm{~mm}$ continuum emission is nonthermal, then any nonthermal contribution at $880 \mu \mathrm{m}$ would be less than $10 \%$ of the total.

\section{A7. Arp 55 (UGC 4881, VV 155)}

Arp 55 is one of the least studied galaxies in our sample. Veilleux et al. (1995) classify the optical spectrum as H II for both components. Lonsdale et al. (1993) detected Arp 55 using VLBI observations, but find the source to be consistent with a compact starburst or a group of clustered supernova.

\section{A8. Arp 193 (IC 883, VV 821, UGC 8387, I Zw 56)}

Veilleux et al. (1995) classify the optical spectrum as LINER. 8.4 GHz images from the VLA show disklike extended emission with no sign of a compact source (Condon et al. 1991). Rush et al. (1996) give an upper limit on the soft X-ray luminosity. Near- and midinfrared images also show disklike extended emission (Scoville et al. 2000; Soifer et al. 2001).

$$
\text { A9. NGC } 6240 \text { (IC 4625, UGC 10592, VV 617) }
$$

Veilleux et al. (1995) classify the optical spectrum as LINER. However, hard X-ray imaging has revealed two AGNs separated by $\sim 1^{\prime \prime}$ (Komossa et al. 2003). Armus et al. (2007) detect continuum emission from dust hotter than $300 \mathrm{~K}$, which also indicates the 
presence of an AGN. High-resolution radio images from 2.3 to $8.4 \mathrm{GHz}$ reveal two compact radio sources with properties similar to Seyfert nuclei (Gallimore \& Beswick 2004). Near-infrared images also show two nuclei as well as bright extended emission (Scoville et al. 2000). For a more complete discussion of the multiwavelength properties of NGC 6240, see Iono et al. (2007).

\section{A10. VV 114 (Arp 236, IC 1623)}

Veilleux et al. (1995) classify the optical spectrum as $\mathrm{H}$ II for both components. $8.4 \mathrm{GHz}$ images from the VLA show no sign of a compact source (Condon et al. 1991). However, Le Floc'h et al. (2002) find that the mid-infrared spectrum shows signs of an AGN in the eastern component. Near and mid-infrared images show extended emission in both components with a bright compact source in the eastern component (Scoville et al. 2000; Soifer et al. 2001).

The millimeter continuum data are consistent with pure dust emission, although the $1.3 \mathrm{~mm}$ continuum point for IC 694 is on the large side of what is possible, leaving open the possibility of a small nonthermal contribution at $880 \mu \mathrm{m}$.

\section{A11. NGC 5331 (UGC 8774, VV 253)}

This galaxy has been relatively poorly studied. Ashby et al. (1995) classify both components as starbursts based on optical spectroscopy and Rush et al. (1996) give an upper limit on the soft X-ray luminosity. Condon et al. (1990) detect both components at $1.49 \mathrm{GHz}$, with the southern component being about twice as strong as the northern component.

\section{A12. NGC 2623 (Arp 243, UGC 4509)}

Heckman et al. (1983) classify the optical spectrum as LINER. 8.4 GHz images from the VLA show a strong compact radio source (Condon et al.1991). Maiolino et al. (2003) classified this galaxy as an obscured AGN using X-ray observations from Chandra. Nearand mid-infrared images show a single compact nucleus (Scoville et al. 2000; Soifer et al. 2001).

The combination of the $1.3 \mathrm{~mm}$ continuum flux from Table 3 with the $880 \mu \mathrm{m}$ continuum flux from this paper suggests that some small nonthermal component may be present. If we assume the nonthermal emission scales inversely with frequency and that the dust emissivity goes as $\beta=1.5$, then any nonthermal contribution at $880 \mu \mathrm{m}$ would be less than $10 \%$ of the total.

\section{A13. NGC 5257, NGC 5258 (Arp 240, VV 55, UGC 8641, UGC 8645)}

Veilleux et al. (1995) classify the optical spectrum as H II and Rush et al. (1996) give an upper limit on the soft X-ray luminosity of NGC 5258. 8.4 GHz images from the VLA show emission in both components that is somewhat extended (Condon et al. 1991). Smith et al. (2007) present Spitzer mid-infrared images at 3.6, 8, and $24 \mu \mathrm{m}$, which show that the bright arm seen in CO in NGC 5258 is also prominent at $24 \mu \mathrm{m}$.

NGC 5258 is an unusual case in that most of the $\mathrm{CO} J=3-2$ emission seen by the SMA comes from the bright southern arm, with only weak emission seen from the nucleus. Surprisingly, this galaxy contains the strongest continuum source in our entire sample and is detected at the $6 \sigma$ level despite a relatively high noise in the SMA image. However, this point source is not located at the center of the galaxy but rather $\left(-15^{\prime \prime}, 8^{\prime \prime}\right)$ to the northwest. Thus, the low gas-to-dust ratio seen in Table 6 is most likely spurious.

\section{A14. NGC 1614 (Arp 186, Mrk 617)}

Veilleux et al. (1995) classify the optical spectrum of NGC 1614 as H II, although the [N II] lines suggest a LINER classification. Neff et al. (1990) find no direct evidence for an AGN component in their 4.6 GHz maps. Fritz et al. (2006) fit the infrared spectral energy distribution with a type II AGN model; however, these model fits suggest that starburst emission is likely the dominant component at $880 \mu \mathrm{m}$. High-resolution radio, near-infrared, and mid-infrared imaging reveal a star-forming ring with diameter $1.2^{\prime \prime}$ (Soifer et al. 2001; Alonso-Herrero et al. 2001; Neff et al. 1990).

NGC 1614 is the only object in our sample besides Mrk 231 to show clear evidence of a nonthermal component from the ratio of its millimeter continuum fluxes. The peak $880 \mu \mathrm{m}$ and $1.3 \mathrm{~mm}$ continuum fluxes are almost identical (Tables 3 and 7 ). If we assume that any nonthermal component depends inversely on frequency, then dust emission represents perhaps $60 \%$ of the total $880 \mu \mathrm{m}$ flux and only $40 \%$ of the peak $880 \mu \mathrm{m}$ flux. However, correcting for this putative nonthermal component produces the highest peak gas-todust ratio of any galaxy in our sample (Table 7).

\section{REFERENCES}

Aalto, S., Radford, S. J. E., Scoville, N. Z., \& Sargent, A. I. 1997, ApJ, 475, L107

Akritas, M. G., \& Siebert, J. 1996, MNRAS, 278, 919

Alonso-Herrero, A., Engelbracht, C. W., Rieke, M. J., Rieke, G. H., \& Quillen, A. C. 2001, ApJ, 546, 952

Alonso-Herrero, A., Rieke, G. H., Rieke, M. J., \& Scoville, N. Z. 2000, ApJ, 532,845

Aretxaga, I., Hughes, D. H., Chapin, E. L., Gaztanaga, E., Dunlop, J. S., \& Ivison, R. J. 2003, MNRAS, 342, 759

Aretxaga, I., Hughes, D. H., \& Dunlop, J. S. 2005, MNRAS, 358, 1240

Armus, L., et al. 2004, ApJS, 154, 178 2007, ApJ, 656, 148

Ashby, M. L. N., Houck, J. R., \& Matthews, K. 1995, ApJ, 447, 545
Baan, W. A., \& Klöckner, H.-R. 2006, A\&A, 449, 559

Blain, A. W., Smail, I., Ivison, R. J., Kneib, J.-P., \& Frayer, D. T. 2002, Phys. Rep., 369, 111

Braine, J., Lisenfeld, U., Duc, P.-A., et al. 2004, A\&A, 418, 419

Bryant, P. M., \& Scoville, N. Z. 1996, ApJ, 457, 678 1999, AJ, 117, 2632

Carico, D. P., Keene, J., Soifer, B. T., \& Neugebauer, G. 1992, PASP, 104, 1086 Chakrabarti, S., Cox, T. J., Hernquist, L., Hopkins, P. F., Robertson, B., \& Di Matteo, T. 2007, ApJ, 658, 840

Condon, J. J. 1992, ARA\&A, 30, 575

Condon, J. J., Helou, G., Sanders, D. B., \& Soifer, B. T. 1990, ApJS, 73, 359

Condon, J. J., Huang, Z.-P., Yin, Q. F., \& Thuan, T. X. 1991, ApJ, 378, 65

Conselice, C. J., Chapman, S. C., \& Windhorst, R. A. 2003, ApJ, 596, L5 
Cox, T. J., Dutta, S. N., Di Matteo, T., Hernquist, L., Hopkins, P. F., Robertson, B., \& Springel, V. 2006, ApJ, 650, 791

Cox, T. J., Primack, J., Jonsson, P., \& Somerville, R. S. 2004, ApJ, 607, L87

Dame, T. M., Hartmann, D., \& Thaddeus, P. 2001, ApJ, 547, 792

Darling, J. 2007, ApJ, 669, L9

Darling, J., \& Giovanelli, R. 2002, ApJ, 572, 810

Dasyra, K. M., et al. 2006, ApJ, 651, 835

Davies, R. I., Tacconi, L. J., \& Genzel, R. 2004, ApJ, 613, 781

Di Francesco, J., Johnstone, D., Kirk, H., MacKenzie, T., \& Ledwosinska, E. 2008, ApJS, 175, 277

Downes, D., \& Solomon, P. M. 1998, ApJ, 507, 615

Draine, B. T., Dale, D. A, Bendo, G., et al. 2007, ApJ, 663, 866

Draine, B. T., \& Lee, H. M. 1984, ApJ, 285, 89

Dumke, M., Nieten, Ch., Thuma, G., Wielebinski, R., \& Walsh, W. 2001, A\&A, 373, 853

Dunne, L., Eales, S., Edmunds, M., Ivison, R., Alexander, P., \& Clements, D. L. 2000, MNRAS, 315, 115

Farrah, D., et al. 2007, ApJ, 667, 149

Frayer, D. T., et al. 1998, ApJ, 506, L7 1999, ApJ, 514, L13

Fritz, J., Franceschini, A., \& Hatziminaoglou, E. 2006, MNRAS, 366, 767

Gallagher, S. C., Brandt, W. N., Chartas, G., Barmire, G. P., \& Sambruna, R. M. 2002, ApJ, 569, 655

Galliano, F., Madden, S. C., Jones, A. P., Wilson, C. D., Bernard, J.-P., \& Le Peintre, F. 2003, A\&A, 407, 159

Gallimore, J. F., \& Beswick, R. 2004, AJ, 127, 239

Gao, Y., \& Solomon, P. M. 2004, ApJS, 152, 63

García-Marín, M., Colina, L., Arribas, S., Alonso-Herrero, A., \& Mediavilla, E. 2006, ApJ, 650, 850

Genzel, R., et al. 1998, ApJ, 498, 579

Goldreich, P., \& Kwan, J. 1974, ApJ, 189, 441

Greve, T. R., et al. 2005, MNRAS, 359, 1165

Graciá-Carpio, J., García-Burillo, S., Planesas, P., \& Colina, L. 2006, ApJ, 640, L135

Greve, T. R., Papadopoulos, P. P., Gao, Y., \& Radford, S. J. E. 2007, ApJ, submitted (astro-ph/0610378)

Gottlöber, S., Klypin, A., \& Kravtsov, A. V. 2001, ApJ, 546, 223

Heckman, T. M., van Breugel, W., Miley, G. K., \& Butcher, H. R. 1983, AJ, 88, 1077

Henning, Th., Michel, B., \& Stignienko, R. 1995, Planet. Space Sci., 43, 1333

Higdon, S. J. U., Armus, L., Higdon, J. L., Soifer, B. T., \& Spoon, H. W. W. 2006, ApJ, 648, 339

Hinz, J. L., \& Rieke, G. H. 2006, ApJ, 646, 872

Ho, P. T. P., Moran, J. M., \& Lo, K. Y. 2004, ApJ, 616, L1

Hughes, D. H., et al. 1993, MNRAS, 263, 607

Imanishi, M., \& Nakanishi, K. 2006, PASJ, 58, 813

Imanishi, M., Nakanishi, K., Tamura, Y., Oi, N., \& Kohno, K. 2007, AJ, 134, 2366

Imanishi, M., Terashima, Y., Anabuki, N., \& Nakagawa, T. 2003, ApJ, 596, L167

Iono, D., Yun, M. S., \& Ho, P. T. P. 2005, ApJS, 158, 1

Iono, D., et al. 2004, ApJ, 616, L63

-. 2007, ApJ, 659, 283 2008, ApJ, submitted

Johnstone, D., Wilson, C. D., Moriarty-Schieven, G., Joncas, G., Smith, G., \& Fich, M. 2000, ApJ, 545, 327

Juvela, M. 1997, A\&A, 322, 943

Juvela, M., \& Padoan, P. 2003, A\&A, 397, 201

Kennicutt, R. C. 1998, ARA\&A, 36, 189

Klaas, U., et al. 2001, A\&A, 379, 823

Komossa, S., Burwitz, V., Hasinger, G., Predehl, P., \& Kaastra, J. S.; Ikebe, Y. 2003, ApJ, 582, L15

Le Fèvre, O., Abraham, R., Lilly, S. J., et al. 2000, MNRAS, 311, 565

Le Floc'h, E., Charmandaris, V., Laurent, O., Mirabel, I. F., Gallais, P., Sauvage, M., Vigroux, L., \& Cesarsky, C. 2002, A\&A, 391, 417

Lonsdale, C. J., Lonsdale, C. J., Smith, H. E., \& Diamond, P. J. 2003, ApJ, 592, 804

Lonsdale, C. J., Smith, H. J., \& Lonsdale, C. J. 1993, ApJ, 405, L9
Maiolino, R., et al. 2003, MNRAS, 344, L59

Marshall, J. A., Herter, T. L., Armus, L., Charmandaris, V., Spoon, H. W. W., Bernard-Salas, J., \& Houck, J. R. 2007, ApJ, 670, 129

Meier, D. S., \& Turner, J. L. 2001, ApJ, 551, 687

Mihos, J. C., \& Hernquist, L. 1996, ApJ, 464, 641

Mundell, C. G., Ferruit, P., \& Pedlar, A. 2001, ApJ, 560, 168

Murphy, T. W., Jr., Armus, L., Matthews, K., et al. 1996, AJ, 111, 1025

Murphy, T. W., Jr., Soifer, B. T., Matthews, K., \& Armus, L. 2001, ApJ, 559, 201

Neff, S. G., Hutchings, J. B., Stanford, S. A., \& Unger, S. W. 1990, AJ, 99, 1088

Neff, S. G., Ulvestad, J. S., \& Teng, S. H. 2004, ApJ, 611, 186

Neri, R., et al. 2003, ApJ, 597, L113

Papadopoulos, P. P. 2007, ApJ, 656, 792

Ptak, A., Heckman, T., Levenson, N. A., Weaver, K., \& Strickland, D. 2003, ApJ, 592, 782

Regan, M. W., et al. 2004, ApJS, 154, 204

Rigopoulou, D., Lawrence, A., \& Rowan-Robinson, M. 1996, MNRAS, 278, 1049

Rosolowsky, E. W., Plambeck, R., Engargiola, G., \& Blitz, L. 2003, ApJ, 599, 258

Rush, B., Malkan, M. A., Fink, H. H., \& Voges, W. 1996, ApJ, 471, 190

Sakamoto, K., Ho,. P. T. P., Iono, D., et al. 2006, ApJ, 636, 685

Sakamoto, K., Scoville, N. Z., Yun, M. S., Crosas, M., Genzel, R., \& Tacconi, L. J. 1999, ApJ, 514, 68

Sanders, D. B., Mazzarella, J. M., Kim, D.-C., Surace, J. A., \& Soifer, B. T. 2003, AJ, 126, 1607

Sanders, D. B., \& Mirabel, I. F. 1996, ARA\&A, 34, 749

Sanders, D. B., Scoville, N. Z., \& Soifer, B. T. 1991, ApJ, 370, 158

Sanders, D. B., Soifer, B. T., Elias, J. H., Madore, B. F., Matthews, K., Neugebauer, G., \& Scoville, N. Z. 1988a, ApJ, 325, 74

Sanders, D. B., Soifer, B. T., Scoville, N. Z., \& Sargent, A. I. 1988b, ApJ, 324, L55

Sault, R. J., Teuben, P. J., \& Write, M. C. H. 1995, in ASP Conf. Proc. 77, Astronomical Data Analysis Software and Systems IV, ed. R. A. Shaw, H. E. Payne, \& J. J. E. Hayes (San Francisco: ASP), 433

Scoville, N. Z., Sanders, D. B., Sargent, A. I., Soifer, B. T., \& Tinney, C. G. 1989, ApJ, 345, L25

Scoville, N. Z., Sargent, A. I., Sanders, D. B., \& Soifer, B. T. 1991, ApJ, 366, L5

Scoville, N. Z., \& Solomon, P. M. 1974, ApJ, 187, L67

Scoville, N. Z., et al. 2000, AJ, 119, 991

Seaquist, E. R., Yao, L., Dunne, L., \& Cameron, H, 2004, MNRAS, 349, 1428

Smith, H. E., Lonsdale, C. J., \& Lonsdale, C. J. 1998, ApJ, 492, 137

Smith, B. J., Struck, C., Hancock, M., Appleton, P. N., Charmandaris, V., \& Reach, W. T. 2007, AJ, 133, 791

Soifer, B. T., Boehmer, L., Neugebauer, G., \& Sanders, D. B. 1989, AJ, 98, 766

Soifer, B. T., et al. 2000, AJ, 119, 509

2001, AJ, 122, 1213

Solomon, P. M., Downes, D., Radford, S. J. E., \& Barrett, J. W. 1997, ApJ, 478, 144

Strong, A. W., \& Mattox, J. R. 1996, A\&A, 308, L21

Strong, A. W., et al. 1988, A\&A, 207, 1

Tacconi, L., Genzel, R., Tecza, M., Gallimore, J. F., Downes, D., \& Scoville, N. Z. 1999, ApJ, 524, 732

Tacconi, L., et al. 2006, ApJ, 640, 228

Tran, Q. D., et al. 2001, ApJ, 552, 527

Veilleux, S., Kim, D.-C., \& Sanders, D. B. 2002, ApJS, 143, 315

Veilleux, S., Kim, D.-C., Sanders, D. B., Mazzarella, J. M., \& Soifer, B. T. 1995, ApJS, 98, 171

Wang, J., et al. 2004, ApJ, 616, L67

Wilson, C. D., \& Scoville, N. Z. 1990, ApJ, 363, 435

Wilson, C. D., Walker, C. E., \& Thornley, M. D. 1997, ApJ, 483, 210

Xia, Y. Y., Xue, S. J., Mao, S., Boller, Th., Deng, Z. G., \& Wu, H. 2002, ApJ, 564, 196

Yang, M., \& Phillips, T. 2007, ApJ, 662, 284

Yao, L., Seaquist, E. R., Kuno, N., \& Dunne, L. 2003, ApJ, 588, 771

Yun, M. S., \& Carilli, C. R. 2002, ApJ, 568, 88

Yun, M. S., Scoville, N. Z., \& Knop, R. A. 1994, ApJ, 430, L109

Zezas, A., Ward, M. J., \& Murray, S. S. 2003, ApJ, 594, L31 